Foundations and Trends ${ }^{\circledR}$ in

Electronic Design Automation

Vol. 7, No. 4 (2013) 247338

(C) 2013 Y. Kim, Y. Wang, N. Chang, and M. Pedram

DOI: $10.1561 / 1000000035$

\title{
Computer-Aided Design and Optimization of Hybrid Energy Storage Systems
}

\author{
By Younghyun Kim, Yanzhi Wang, \\ Naehyuck Chang, and Massoud Pedram
}

Contents

\begin{tabular}{lll}
\hline 1 & Introduction & 249
\end{tabular}

2 Energy Storage Elements 253

2.1 Performance Metrics for Energy Storage Elements 253

2.2 Energy Storage Elements 259

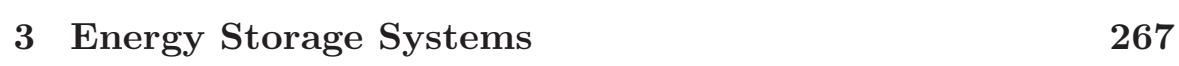

3.1 Architecture of Electrical Energy Storage Systems 268

3.2 Classification of Energy Storage Systems 273

4 Hybrid Energy Storage Systems 282

4.1 Motivation and Principle 282

4.2 System Architecture 286

5 Hybrid Energy Storage System

Design and Optimization 293

\begin{tabular}{|ll}
5.1 & Design-time Optimization 293
\end{tabular}

5.2 Runtime Optimization 298 
6 Hybrid Energy Storage System Applications 310

6.1 Residential/Household Applications 310

6.2 Electric Vehicle and Hybrid Electric

6.3 Low-power Embedded Systems Applications 319

$\begin{array}{|ll|}7 \text { Conclusions } & 323\end{array}$

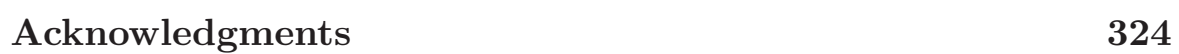

\begin{tabular}{|l|l}
\hline References & 325
\end{tabular} 
Foundations and Trends ${ }^{\circledR}$ in

Electronic Design Automation

Vol. 7, No. 4 (2013) 247338

(C) 2013 Y. Kim, Y. Wang, N. Chang, and M. Pedram

DOI: $10.1561 / 1000000035$

\title{
Computer-Aided Design and Optimization of Hybrid Energy Storage Systems
}

\author{
Younghyun Kim¹, Yanzhi Wang ${ }^{2}$, \\ Naehyuck Chang ${ }^{3}$, and Massoud Pedram ${ }^{4}$ \\ 1 Seoul National University, 1 Gwanak Rd, Gwanak-gu, Seoul, 151-744, \\ Korea,yhkim@elpl.snu.ac.kr \\ 2 University of Southern California, 3740 McClintock Ave, Los Angeles, \\ 90089,USA,yanzhiwa@usc.edu \\ 3 Seoul National University, 1 Gwanak Rd, Gwanak-gu, Seoul, 151-744, \\ Korea,naehyuck@elpl.snu.ac.kr \\ 4 University of Southern California, 3740 McClintock Ave, Los Angeles, \\ 90089,USA, pedram@usc.edu
}

\begin{abstract}
Electricity is the key to the proper functioning of modern human society. Ever-increasing electricity consumption gives rise to recent regulations and significant endeavors to improve the energy efficiency in all kinds of human activity from manufacturing to commerce, from transportation to digital communication, from entertainment to laptops and portable devices. An important technology for helping reduce energy consumption is the ability to store any excess electrical energy for long periods of time and efficiently retrieve the stored energy.

The design and management of electrical energy storage systems is the focus of the present paper, which starts off by reviewing and comparing various types of electrical energy storage elements in terms of
\end{abstract}


various metrics of interest ranging from power and energy density to output power rating and from self-leakage rate to cost per unit of stored energy, and from life cycle of the storage element to the efficiency of the charge/discharge cycle. Next the paper reviews various energy storage systems while motivating the need for a hybrid energy storage system comprised of heterogeneous types of energy storage elements organized in a hierarchical manner so as to hide the weaknesses of each storage element while eliciting their strengths. The paper continues with a detailed explanation of key challenges that one faces when dealing with the optimal design and runtime management of a hybrid energy storage system targeting some specific application scenario; for example, grid-scale energy management, household peak power shaving, mobile platform power saving, and more. A survey of some existing solutions to these problems is also included. 


\section{1}

\section{Introduction}

Electricity is an integral utility in the modern society, with links to everything from agricultural production to manufacturing, from digital communication to media and internet, and from medical care to living conditions. Electric energy consumption has steadily risen since its industrial introduction in the second half of the nineteenth century. In fact the world's total electrical energy production in 2009 was about 20,000 TWh, which is equivalent to a generated (and consumed) power of around $2.3 \mathrm{TW}$ on average. This level of average power consumption is achieved by a combination of electricity generation stations, including heat engines fueled by chemical combustion or nuclear fission, kinetic energy of flowing water and wind, solar photovoltaics and geothermal processes. Fossil fuels (coal, gas, and oil in that order) account for $67 \%$, renewable energy (mainly hydroelectric, wind, solar, and biomass) for $16 \%$, nuclear power for $13 \%$, and other sources for $3 \%$ of all electrical energy produced worldwide. Emissions of pollutants and greenhouse gases from fossil fuel-based electricity generation are responsible for a significant portion of world greenhouse gas emissions. Although Solar PV generation is advertized as environmentally friendly, fabrication of 
PV cells utilizes large amounts of water in addition to releasing toxic chemicals such as phosphorus and arsenic.

Reliable supply of electric energy is also an important issue. Power outage is regarded as a public emergency as people take the availability of uninterrupted power supply for granted. Electrical energy consumption in a system changes over time due to changes in the power requirements of load devices as well as the users' behaviors. Load-following power plants (for example, fossil fuel power plants) are intended to handle rapid changes in power demands on the power grid. In addition, the grid requires a certain level of operating reserve, which is made up of spinning and non-spinning reserves, in order to prevent blackouts and brownouts. Spinning reserve denotes the on-line extra generating capacity to deal with the peak power demand that can arise for a short period of time. Non-spinning reserve, on the other hand, refers to the off-line additional generating capacity that can be turned on and connected to the power grid after a short delay. Both the spinning and non-spinning reserves require extra capital investment by the utility companies for their generation facility setup and operation. Reserve power generation is generally more costly than the normal operation on the power grid. Some countries have only small reserve margin during the peak hours, which threatens the power supply and demand match and gives rise to risky operating reserve guard banding. This can be remedied by building extra power plants. However, construction of new power plants requires large capital investment and has social and environmental costs.

To tackle the high demand for electric power and reduce the power plant over-provisioning, electrical energy storage systems (ESS) have been proposed [31]. An ESS performs operating reserve management, which is performed by expensive, environmentally unfriendly loadfollowing power plants. In addition, the ESS effectively enhances the power grid stability as well as the availability of renewable power sources such as windmills and photovoltaic (PV) panels. Renewable power sources have unreliable power generation characteristics; the level of power generation of the renewable power sources, such as PV cells and windmills, is heavily dependent on environmental factors (for example, the solar irradiance level or climate conditions). The ESS also 
resolves the mismatch between the power generation and power consumption times in case of renewable power sources.

Nevertheless, ESS technologies are not ready for large-scale and widespread deployment. The main reason is that in spite of the large variety of ESS technologies, no technology offers sufficient performance in respect of key figures of merit needed of an electrical energy storage medium. For instance, a high-performance ESS should exhibit high cycle-efficiency, high power and energy storage capacity, low cost, high volumetric and/or graviometric density, and long-cycle life. The ESS technology of choice for many applications (especially those requiring high volumetric and/or graviometric density) is battery storage. Different battery technologies, however, have widely different characteristics. Again no single battery can simultaneously achieve all the desired characteristics of a high-performance ESS. Furthermore, no battery technology is in sight that can achieve these characteristics. So the focus is on finding ways to build ESS that comprise of different battery types so as to hide the weaknesses of each battery type, yet presenting the strongest features of each battery type.

It is a practically promising solution to develop system-level design methodology that enhances storage system performance and lifetime through efficient use of the current energy storage technologies. A hybrid ESS (HESS) consists of multiple heterogeneous energy storage elements so as to exploit the unique advantages of each energy storage element while hiding their unique shortcomings by introducing novel storage system architecture and hierarchy along with sophisticated charge management policy and means [124]. The HESS concept is derived in analogy to the computer memory hierarchy employed in computer systems and used to provide low-latency, yet low-cost, access to program and data storage.

However, designing the optimal HESS is not a trivial problem. Simply mixing different types of energy storage elements does not automatically guarantee to make up a high performance ESS. HESS architectural design is a multi-variable multi-objective optimization, and heterogeneity of the energy storage elements explodes the design complexity. It includes both continuous and discrete design parameters and many complex nonlinear models. Management policies of HESS 
involves in another highly complicated runtime optimization. Therefore, computer-aided design and optimization is a must for the optimal design and operation of the HESS with reasonable time and efforts.

This paper covers a wide range of topics regarding the computeraided design and runtime management of HESS. The remainder of the paper is organized as follows.

- Section 2 begins with the introduction and evaluation of various types of energy storage elements. We review some of the performance metrics for energy storage elements and compare these elements in terms of these metrics.

- Section 3 introduces the ESS architecture and components in more detail and provides an overview of research work focusing on the ESS.

- From Section 4, we focus on the HESS. We first explain the HESS architecture in analogy with the computer memory hierarchy. Next we discuss various flavors of HESS architectures focusing on energy storage hybridization.

- Section 5 reviews some of the recent work on the systematic optimization of the HESS, including both design-time and runtime optimization schemes that maximize the benefits of the HESS.

- Section 6 provides a survey of applications of the HESS, including the power grid, electric vehicle (EV)/hybrid electric vehicle (HEV), and low-power embedded systems.

- Section 7 concludes this paper by outlining possible future directions for the HESS research and development. 


\section{Energy Storage Elements}

An energy storage element is an elemental device or apparatus that is capable of storing electrical energy in the potential, kinetic, chemical, or other forms of energy, and restoring the stored energy back to the electrical energy on demand. There are many types of energy storage elements developed to-date. In this section, we enumerate the key metrics that are frequently used to evaluate different energy storage elements in Section 2.1 in detail. Next we compare some representative energy storage elements in light of the discussed metrics in Section 2.2 We do not discuss the detailed principles behind storing (releasing) the electrical energy in (from) storage elements (for example, internal electro-chemical reactions), but focus on evaluating their performance and cost as energy storage elements.

\subsection{Performance Metrics for Energy Storage Elements}

This section introduces the performance metrics that are used to characterize energy storage elements in Section 2.2. We focus on how various storage elements can be combined in a hybrid manner to hide each other's weaknesses. 


\section{Energy Storage Elements}

\subsubsection{Cycle Efficiency and Internal Resistance}

Cycle efficiency of an energy storage element is defined by the roundtrip energy efficiency, that is, ratio of the amount of energy output during discharging to the energy input during charging. In other words, the cycle efficiency is the product of charging efficiency and discharging efficiency. Here, the charging efficiency is the ratio of energy stored in an energy storage element after charging to the total energy supplied to that element during the entire charging process, and discharging efficiency is the ratio of energy extracted from an energy storage element during the discharging to the total energy before the discharging process begins.

Power loss during the charge and discharge cycles is mostly due to internal resistance of the energy storage elements. Cycle efficiency is drastically affected by charging/discharging profiles, that is, the magnitude and shape of the charging/discharging current. For example, due to the well-known rate capability effect, the total energy delivered by a battery goes down with the increase in load current, resulting in lower discharging efficiency. At the same time, the recovery effect of battery which recovers the terminal voltage during idle periods between current pulses also affects the cycle efficiency of the battery [83].

Supercapacitor and flywheel have a very high cycle efficiency which is close to $100 \%$ and less affected by the charging/discharging profile. A high cycle efficiency means less energy loss during charging and discharging processes which leads to low operational cost per each cycle. Therefore, it is wise to use a high-cycle efficiency energy storage element such as supercapacitor for frequent charging/discharging applications.

\subsubsection{Rate Capability}

Rate capability (also referred to as the power capability and sometimes called the rate capacity) signifies the capability to provide high power without degradation of total amount of energy. A battery's available capacity decreases as the discharging rate increases. Peukert's law describes the phenomenon that the delivered capacity normalized to the rated capacity has an exponential relationship to the discharge current 
normalized to the rated current [43]. That is,

$$
I^{k} \cdot t=\text { fixed_capacity }
$$

where $I$ is the discharge current, $t$ is discharge time, and $k$ is the Peukert constant, which is typically a value between 1 and 2. Generally, the Peukert constant varies according to the age of the battery, generally increasing with age. In an ideal battery where the total delivered capacity is independent of the discharge current, $k=1$. For a lead-acid battery $k$ is between 1.1 and 1.3. In contrast, supercapacitors have a very good rate capability and can withstand very high discharge rates with virtually no loss of available capacity (Figure 2.1).

Note that if $I$ is described as actual discharge current relative to 1 ampere, then $I^{k} \cdot t$ will give the battery capacity at a one-ampere discharge rate. This is another common form of the Peukert's law.

\subsubsection{Energy Density versus Power Density}

Power density is defined as the rated output power divided by volume $(\mathrm{W} / \mathrm{L})$ or mass $(\mathrm{W} / \mathrm{kg})$ of the energy storage element. Similarly, energy density is the stored energy divided by the volume (Wh/L) or mass (Wh/kg). Generally speaking, power density is related to the amount of instantaneous power that an ESS element can provide; on the other hand, energy density is related to the time duration that the energy storage element can last while supplying a certain amount of power.

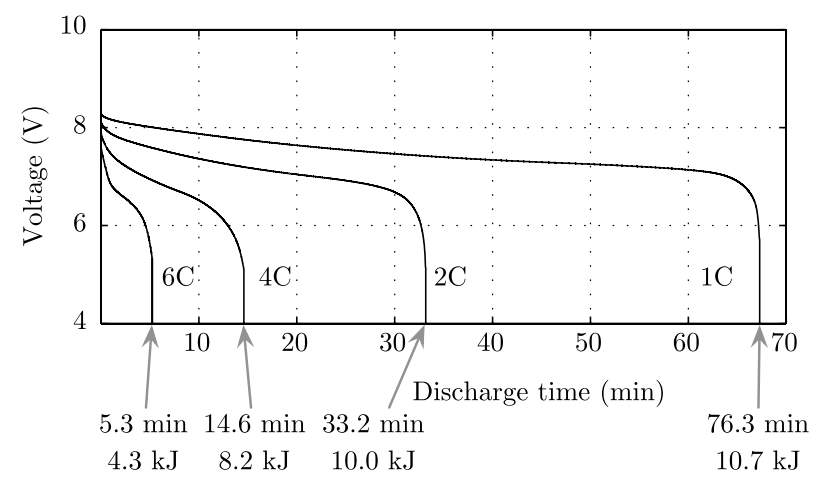

Fig. 2.1 Discharging a $350 \mathrm{mAh}$ 2-cell series lithium-ion GP1051L35 battery at a constant current of $1 \mathrm{C}, 2 \mathrm{C}, 4 \mathrm{C}$, and $6 \mathrm{C}$ [139. 
Supercapacitor and flywheel have a very high power density, whereas typical lithium-ion batteries have a marginally high power density. High-power density energy storage elements are suitable as temporary energy buffers to deal with short-duration high-power demand of some applications. Metal-air batteries and fuel cells have a high-energy density, which is multiple orders of magnitude higher than those of typical batteries. High-energy density energy storage elements are suitable as long-term energy storage means. Supercapacitor is one of the energy storage elements that has the worst energy density, and so it is not economical to use them alone as a large-scale energy storage means.

A HESS can rely on high-energy density energy storage elements for long-term storage and a relatively small amount of high-power density energy storage elements for high output power rating. Batterysupercapacitor hybrid is a representative HESS, which exploits the high-energy density of the battery and high-power density of the supercapacitor. We discuss the battery-supercapacitor HESS in Section 3.2 .1 .2 in more detail.

\subsubsection{Capital Cost}

Capital cost, which is an important consideration in the design and implementation of an ESS, is typically represented in the forms of cost per unit of delivered energy $(\$ / W h)$ or per unit of output power $(\$ / W)$. The capital cost determines how much money should be invested in order to build an ESS with certain amount of energy capacity or power capacity.

Some batteries are superior to others in terms of both their energy and power densities (for example, lithium-ion battery is better than lead-acid in both respects), yet the cost of these batteries are quite different with lead-acid having much lower cost. We should thus carefully determine the portion of expensive (but higher performance) energy storage elements and inexpensive (but lower performance) energy storage elements for a given monetary budget. In fact, the capital cost gap between energy storage elements is a key motivation for the hybrid storage approach because one cannot afford to use unlimitedly large 
amount of good, yet expensive, energy storage elements to meet the ESS requirements. For example, we can use a very large amount of supercapacitors to meet both the high output power requirement and the high-energy storage requirement of some applications, but the cost of such an ESS will be impractically high. Instead, consider an alternative ESS that uses a small amount of supercapacitors to meet the high instantaneous power demand and a small amount of lithium-ion batteries to meet the high storage requirement of the same applications. The cost will be affordable.

Some previous research attempt to minimize the cost of HESS. A sizing optimization method for stand-alone photovoltaic power systems utilizing a HESS is presented in [90]. The optimization goal is to find cost-optimal combination of photovoltaic power generation module, fuel cell, and battery. Control strategies for optimization of system performance and cost in a battery and fuel cell HESS are studied in [151].

\subsubsection{Cycle Life and State-of-health (SoH)}

State-of-health (SoH) of an energy storage element is a measure of its age. It captures the general condition of the energy storage element and its ability to store and deliver energy compared to its initial state (that is, when it was fresh device out of the manufacturing line). During the lifetime of the energy storage element, its capacity (or "health") gradually deteriorates due to irreversible physical and chemical processes that take place along with usage. Cycle life is the maximum number of charging and discharging processes that an energy storage element can perform before its capacity drops to a specific percentage $(60 \%-80 \%$ typically) of its initial capacity. It is one of the key performance parameters, which gives an indication of the expected working lifetime of the energy storage element. Obviously, the cycle life of an energy storage element is closely related to the replacement period for it and thus the full cost of the element according to a life-cycle analysis.

Cycle life of an energy storage element heavily depends on the usage pattern of the element, especially to the depth-of-discharge (DoD) which is defined as ratio of used capacity to the initial full capacity. For lead-acid batteries, the number available charging/discharging cycles 
increases when lowering the DoD 61]. Similar conclusions hold for most electrochemical batteries.

Typically, energy storage elements whose operation principles are based on electrical, mechanical or thermal technologies, such as supercapacitor, flywheel, thermal energy storage (TES), cryogenic energy storage (CES), typically have long cycle lives. In contrast, cycle life of electrochemical batteries are not that high due to unavoidable chemical deterioration of the electrodes during their operation.

\subsubsection{Self-discharge Rate}

Self-discharge rate is a measure of how quickly a storage element loses its stored energy when there are no charging and discharging currents. It is heavily dependent on how the energy storage element stores the energy inside, as well as ambient conditions such as the temperature and humidity. Supercapacitor and flywheel, which have a high cycle life, have highest self-discharge rate, that is, they typically lose all their stored energy within a few days, or even hours. One should thus not store energy in a supercapacitor if it is expected that the energy will not be used for a long time. On the other hand, electrochemical batteries store the energy with stable chemicals, and do not lose so much energy by themselves.

\subsubsection{Environmental Impacts}

The importance of environmental friendliness of energy storage elements is being emphasized starting the recent past. Typically, electrochemical batteries have a negative impact on the environment due to their reliance on toxic metals such as lead and cadmium that can be quite harmful if not disposed of properly. They should be recycled properly for both reducing environmental impact and conserving scarce resources [170]. The environmental impacts of an energy storage element are closely related to its expected cycle life. In other words, we can reduce the negative impacts of using hazardous energy storage elements by extending their cycle lives. Note that the supercapacitor and flywheel have very small or almost negligible impacts on the 
environments, not only because they no not contain harmful materials, but also because of their long cycle lives.

\subsection{Energy Storage Elements}

\subsubsection{Batteries}

Lithium-ion battery Lithium-ion battery, first demonstrated in the 1970s, is a family of rechargeable batteries in which lithium ions move from a negative electrode to a positive electrode through discharging. The ions move in opposite direction during the charging process. Lithium-ion is now the battery of choice in portable electronic devices and is growing in popularity in mobile systems ranging from EV to airplanes. The growing popularity of lithium-ion battery is mainly due to the following reasons: high-energy density, high efficiency, long cycle life, no memory effect, and low self discharge rate.

While commanding a 50\% share of the small portable devices market [31, there are many challenges to build large-scale lithium-ion based ESS. The main hurdle is the high cost due to special packaging and internal overcharge protection circuitry. There are also concerns about their safety and fire risk. Manufactures are working to reduce the manufacturing cost of lithium-ion batteries to capture large new energy markets, especially markets for the electrical vehicles. At the same time, many are working on making large-size lithium-ion cells safer and more stable. Figure 2.3(a) shows a 18650 size lithium-ion battery, ICR18650-22F from Samsung SDI. Multiple lithium-ion batteries of this size are combined in a pack and are widely used for portable devices such as laptops.

New materials such as graphene and silicon thin film are under development to improve the energy density and cycle life of the lithiumion batteries as shown in Figure 2.2 [3, 18, 19, 20, 54, [57, 92, 152, 153, [171, 176].

Estimating the state-of-charge ( $\mathrm{SoC})$, which is the remaining energy, and $\mathrm{SoH}$ of batteries is important for system management, but it is not an easy task 131. Significant number of research have been performed to accurately estimate the $\mathrm{SoC}$ and $\mathrm{SoH}$ by developing accurate models because simply measuring voltage and current does not give an good 


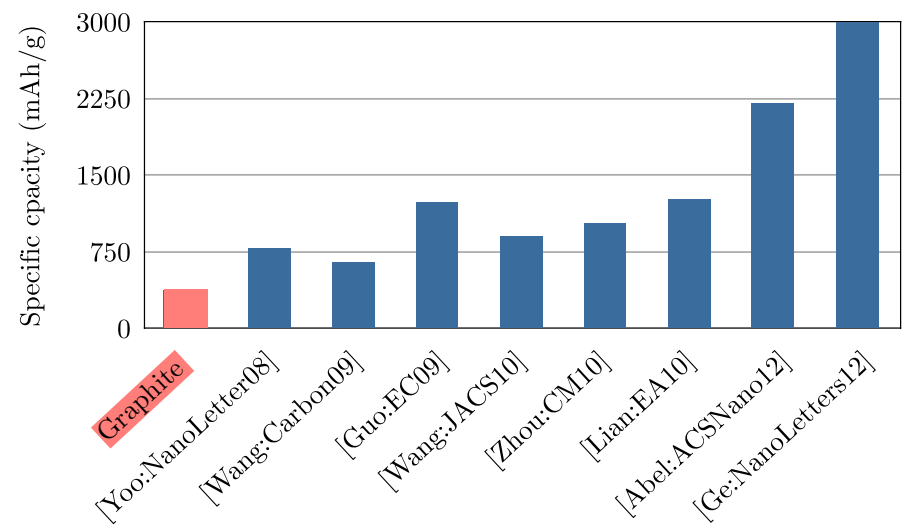

Fig. 2.2 Improved specific capacity with respect to the electrode weight of lithium-ion batteries with new electrode materials.

estimation. A lithium-ion battery circuit model is proposed for developing a battery-supercapacitor hybrid in [53]. A dual extended Kalman filter is used to simultaneously estimate the SoC and capacity from the open-circuit voltage [86]. A lithium-ion battery model introduced in 22] employs the method of electrochemical impedance spectroscopy.

Lead-acid battery As one of the oldest and most developed rechargeable battery technologies, lead-acid batteries have short cycle life and low-energy density due to the inherent high density of lead as a metal. Besides, they also have a poor low temperature performance and thus a thermal management system is required. In spite of these disadvantages, their ability to supply high surge currents means that such lead-acid batteries maintain a relatively high-power density. These features, along with their low cost and high-energy efficiency, make lead-acid batteries suitable in motor vehicles so as to provide the high current demand for automobile starter motors. Lead-acid batteries have also been used in a few large-scale commercial energy management systems. Figure 2.3(b) shows an example of a valve-regulated (sealed) leadacid battery pack, Panasonic LC-R123R4P. Lead-acid battery technology is considered quite mature now [31, but there are still efforts to find better materials for the current collectors, electrodes, electrolytes, etc [39, 119]. 


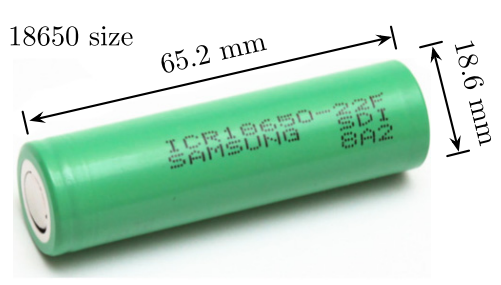

Samsung SDI ICR18650-22F

Nominal voltage: $3.7 \mathrm{~V}$

Rated capacity: $2.2 \mathrm{Ah}$

(a) Li-ion battery

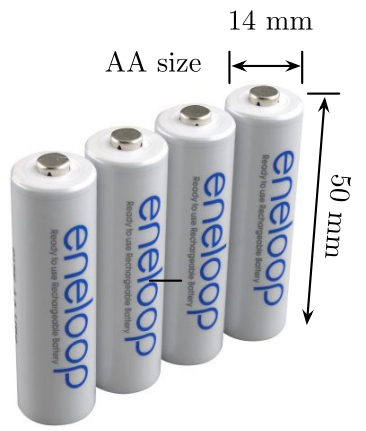

Sanyo HR-3UTGA

Nominal voltage: $1.2 \mathrm{~V}$

Rated capacity: $2.0 \mathrm{Ah}$

(c) NiMH battery

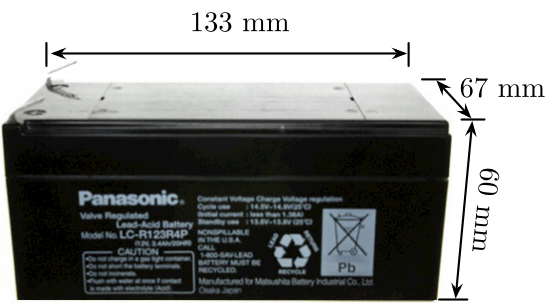

Panasonic LC-R123R4P

Nominal voltage: $12 \mathrm{~V}$

Rated capacity: 3.4 Ah (20 hour rate)

(b) Lead-acid battery

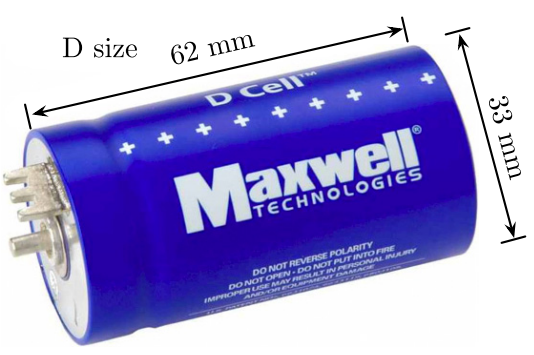

Maxwell Technologies BCAP0310

Capacitance: $310 \mathrm{~F}$

Rated voltage: $2.7 \mathrm{~V}$

Rated capacity: $0.31 \mathrm{Wh}$

(d) Supercapacitor

Fig. 2.3 Example commercial products of typical batteries and supercapacitor.

Nickel-metal hydride batteries Nickel-metal hydride battery, abbreviated NiMH, is a type of rechargeable battery similar to the nickel-cadmium cells (NiCd). The only difference is that the former one uses a hydrogen-absorbing alloy for the negative electrode instead of cadmium. The energy density of NiMH batteries is more than double that of lead-acid batteries and $40 \%$ higher than that of $\mathrm{NiCd}$ batteries. NiMH battery is relatively inexpensive. However, it suffers from the memory effect, although this is much less pronounced than that in the NiCd ones. NiMH battery also has a rather high 
self-discharge rate. Recently, low self-discharge (LSD) NiMH battery have been commercialized targeting portable devices. Figure 2.3(c) is a commercial product of an AA size LSD NiMH battery from Sanyo. Toyota Prius HEV also uses NiMH batteries [182.

The most significant feature of NiMH batteries is the high power density $(250-1,000 \mathrm{~W} / \mathrm{kg}$ ), which is the highest among existing battery types. Therefore these batteries are widely used in high current drain consumer electronics, such as digital cameras with LCDs and flashlights. Recently, NiMH batteries have also been deployed in HEV such as the Toyota Prius, Honda Insight, Ford Escape Hybrid, Chevrolet Malibu Hybrid, and Honda Civic Hybrid.

\subsubsection{Capacitors and Supercapacitors}

Electric double-layer capacitors, more commonly known as supercapacitors or ultra capacitors, are widely exploited to mitigate load current fluctuations in the batteries. Supercapacitors have a superior cycle efficiency that reaches almost 100\%, and a long cycle life [142. Moreover, compared with batteries, supercapacitors exhibit significantly higher volumetric power density (but they have much lower energy density) 9. Therefore, they are suitable as energy storage in situations with frequent charging/discharging cycles or periodic high current pulses. In a battery-supercapacitor hybrid system, the supercapacitor stores surplus energy from the battery during low demand periods, and provides extra energy during peak load current demand period.

A key disadvantage of a supercapacitor is its large self-discharge rate compared to that of ordinary batteries. A supercapacitor may lose more than $20 \%$ of its stored energy per day even if no load is connected to it. Another important concern with respect to supercapacitors is their terminal voltage variation, which in turn arises from the characteristics of any capacitor whereby its terminal voltage is linearly proportional to its SoC. The terminal voltage thus increases or decreases accordingly as the supercapacitor is charged or discharged. This terminal voltage variation is much higher than that observed in typical batteries. This effect results in a significant conversion efficiency variation in the power converters that are connected to the supercapacitors. The HESS including 
supercapacitors should thus carefully account for the above characteristics to be practical.

Figure 2.3(d) is a commercially available supercapacitor, BCAP0310 from Maxwell Technologies. Its size is the same as that of a D size battery, and it has a $310 \mathrm{~F}$ capacity with the maximum voltage of $2.7 \mathrm{~V}$, which results in $0.31 \mathrm{Wh}$ energy capacity. In comparison, a high-energy density D size lithium primary battery has 68.4 Wh energy capacity.

Research efforts are being devoted to develop new materials that enhance the energy density of the supercapacitors as shown in Figure 2.4. Graphene and carbon nanotube (CNT) are being actively pursued as supercapacitor electrodes [4, 19, 32, 33, 67, 96, 112, 150, 160. For example, graphene/CNT composite electrodes introduced in 32 achieves a very high-energy density of $155.6 \mathrm{Wh} / \mathrm{kg}$, which is comparable to that of NiMH batteries.

Accurate characterization and modeling the supercapacitors are important to understand and utilize their benefits because the simple ideal capacitor model does not fully explain their behavior. A physical model of electric double-layer supercapacitors is introduced in [13]. Two branches model in this paper is composed of two resistor-capacitor sets for quick and slow energy response, respectively. Some research focus on impedance characteristics of the supercapacitors, which is important for dynamic behavior [22, 21]. A supercapacitor equivalent electric model in [127] considers the variation of the resistance and capacitance by the temperature and frequency. It also accounts the leakage current and internal charge redistribution.

\subsubsection{Flow Batteries and Flow Capacitors}

A flow battery generates electricity inside a reactor by using dissolved electroactive species stored in external tanks. This separation of reactor and tank decouples the energy density (limited by tank size) from the power density (limited by reactor size). Flow batteries have advantages of long cycle life, environmental friendliness, quick charging by electrolyte replacement, and so on. However, they require complicated components and complex circuitry such as sensors and actuators to 


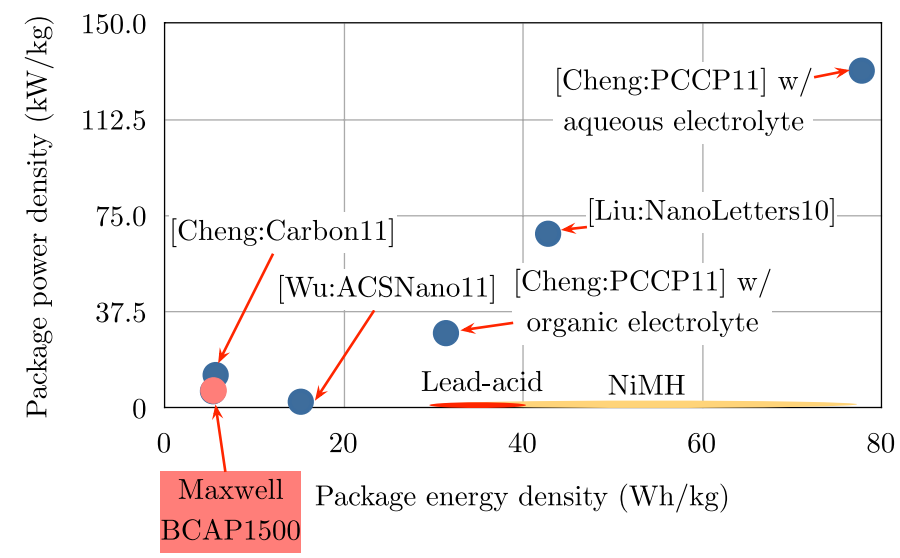

Fig. 2.4 Improved power and energy density of supercapacitors with new electrode materials. Package density is approximated to the half of the density with respect to the electrode weight 96.

run the system. Flow batteries are generally considered for large-scale stationary applications rather than portable applications. Vanadium redox battery [50] and zinc-bromine battery [89] are examples of flow batteries. Lead-acid flow battery is also considered as a replacement of the conventional lead-acid batteries [117, 161, 173].

A recent technology called the electrochemical flow capacitor has the advantages of both supercapacitors and flow batteries [125. It provides rapid charging and discharging, high cycle life, and high cycle efficiency like a supercapacitor. It also provides and high-energy capacity, decoupled with the power capacity, like a flow battery. A flowable carbon-electrolyte mixture, called a slurry, captures or releases charged ions while flowing through a flow cell during a charging or discharging process.

\subsubsection{Other Energy Storage Elements}

There are many other types of energy storage elements such as nickelcadmium $(\mathrm{NiCd})$ battery, sodium-sulfur $(\mathrm{NaS})$ battery, metal-air battery, flywheel, cryogenically cooled thermal plates, and compressed air 


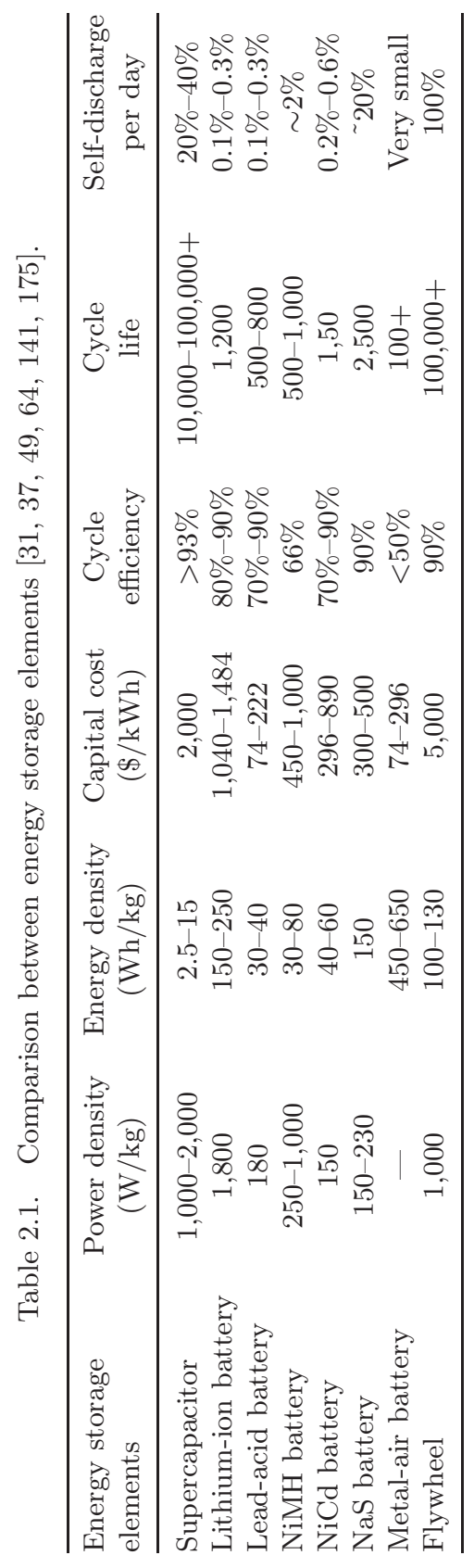



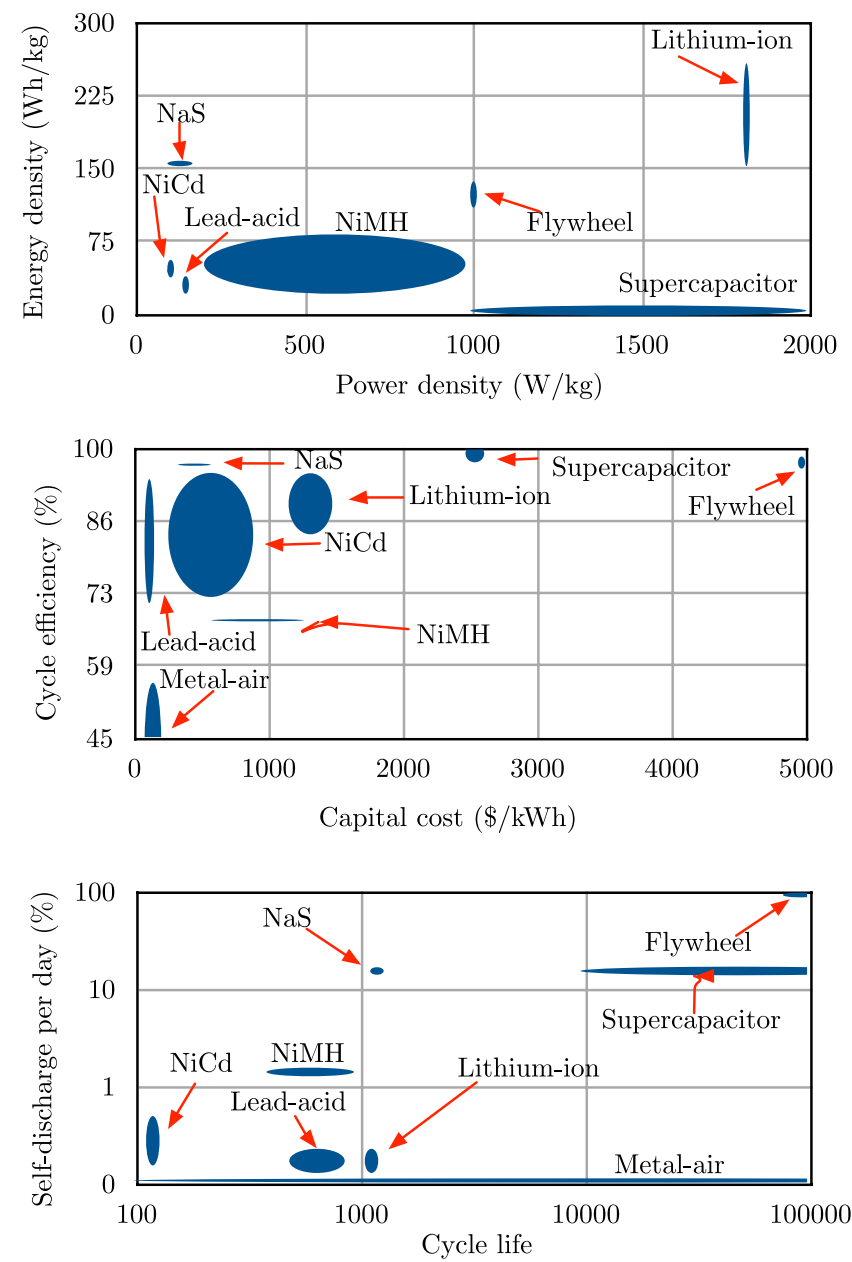

Fig. 2.5 Comparison of energy storage elements [31, 37, 49, 64, 141,175 .

systems. We do not cover all the detail of those energy storage elements in this paper. Characteristics of some of these energy storage elements are summarized in Table 2.1 and Figure 2.5 together with the energy storage elements we discussed above. 


\section{Energy Storage Systems}

An ESS is an energy reservoir which stores electrical energy and supplies the energy when necessary. The electrical energy is stored in the ESS as potential energy, kinetic energy, chemical energy, or in some other form in the energy storage elements that we discussed in Section 2, An ESS performs many useful functions such as load leveling, contingency service, voltage stabilization, maximum power point tracking (MPPT) for renewable power sources, and more, for a wide range of applications including portable devices, household appliances, $\mathrm{EV} / \mathrm{HEV}$, and even power grid.

We first discuss the general architecture and components of the ESS in Section 3.1. Next we provide a classification of the ESS based on the homogeneity vs. heterogeneity of their constituent energy storage elements and potential application (therefore, their size and scale) in Section 3.2 . 


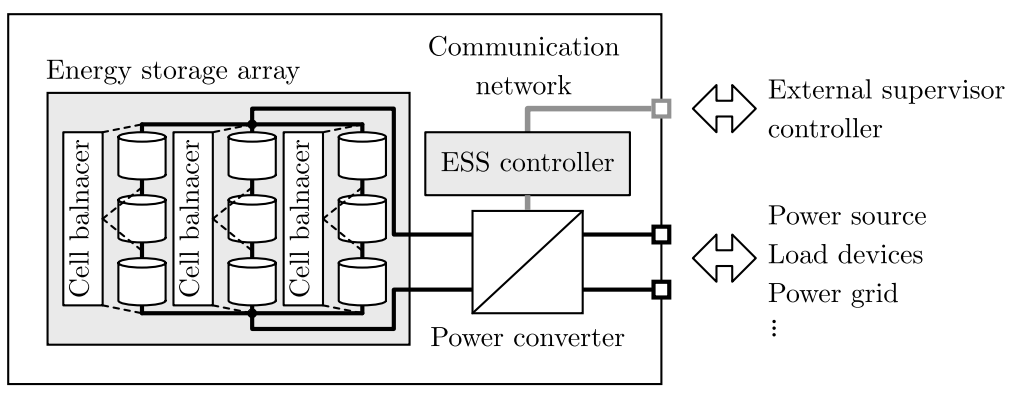

Fig. 3.1 ESS architecture.

\subsection{Architecture of Electrical Energy Storage Systems}

Figure 3.1 shows a typical architecture of an ESS. Major components of an ESS include an energy storage array, power converter, and ESS controller, which we discuss in the following subsections.

\subsubsection{Energy Storage Array}

The energy storage element is the most important consideration for ESS design. There are various types of energy storage elements as we discussed in Section 2.2. Type and capacity energy storage element is determined according to the application and requirement of the ESS with restriction on volume, weight, cost, and so forth.

When a single energy storage element does not meet the energy or power requirement, which is usual for a large-scale ESS, multiple energy storage elements are connected together to form an array of energy storage elements. An energy storage array is a set of multiple identical energy storage elements that are connected in series and/or parallel forming an regular matrix. The dimension of the energy storage array is determined by power and energy capacity requirement, and the maximum voltage rating of the energy storage array. The regular array structure makes it possible to maintain the same $\mathrm{SoC}$ and $\mathrm{SoH}$ of all the elements in the array.

Some types of energy storage elements requires balancing between serially connected cells. Even though all the energy storage elements are of the same type, manufacturing variation in practice may result 


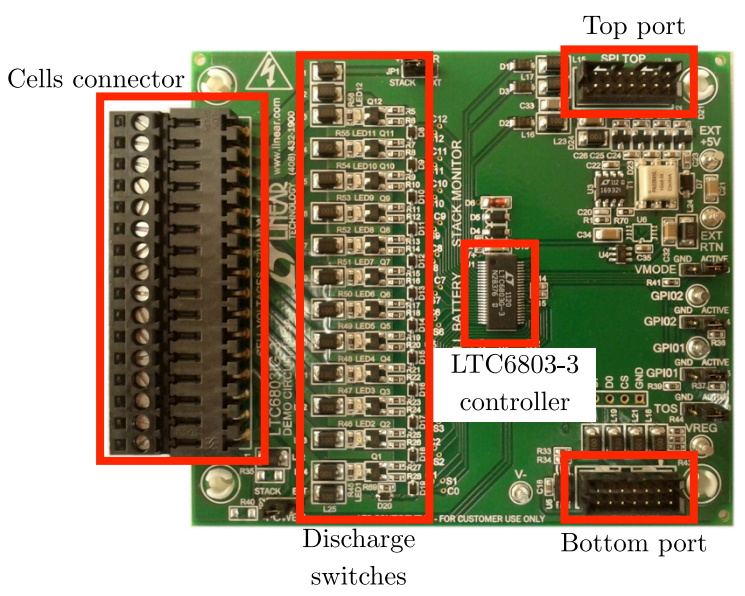

Fig. 3.2 Linear Technology LTC6803-3 battery stack monitor with passive cell balancing switches 94 .

in imbalance of characteristics such as capacity and internal resistance that causes imbalanced SoC during operation and even damage to the elements 11. Supercapacitors and lithium-ion batteries require external cell balancing circuits like Figure 3.2. Cell balancing is an active research area, which is critical for large scale ESS [7, 28, 45, 65]. A paper categorizes cell balancing methods into three categories: charging methods (steering), active methods, and passive methods (bleeding) [110]. The charging method selectively bypasses fully charged cells. The active cell balancing methods moves energy from more-charged cells to less-charged cells my means of capacitors or power converters. The passive cell balancing methods simply dissipate energy from over-charged cell as heat.

Configuration of energy storage elements, that is, number of series and parallel connections, may be dynamically adjusted. For example, with four supercapacitors, three configurations, 1-by-4, 2-by-2, and 4-by-1, are feasible. Supercapacitors, which have wide voltage variation according to SoC, benefit from the dynamic reconfiguration 30. A supercapacitor array maintains roughly constant voltage by increasing (deceasing) number of series connections as the voltage of each energy storage element decreases (increases). Dynamic reconfiguration circuit proposed in [51, 77, 147, 148, 149] aims at maintaining 

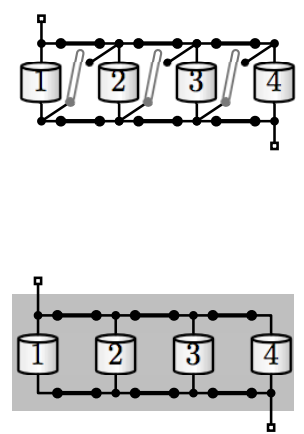

(a) 1-by-4 configuration
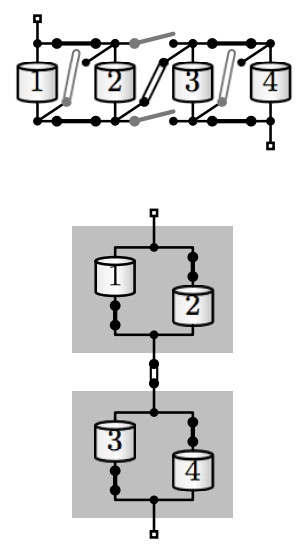

(b) 2-by-2 configuration
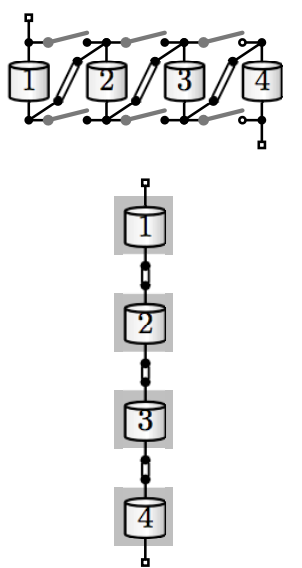

(c) 4-by-1 configuration

Fig. 3.3 Reconfiguration examples of an energy storage array with four energy storage elements [7].

marginally constant supercapacitor array voltage. It is shown that explicitly considering the power converter efficiency further improves the energy efficiency than simply maintaining a constant voltage 77 (Figure 3.3 ).

Fault resilience is another benefit of the dynamic energy storage array reconfiguration. Energy storage elements are subject to manufacturing variations. Each energy storage element shows different aginginduced battery capacity degradation [91, 162]. Dynamic reconfiguration may prolong the lifetime of the energy storage array when short-circuit faults or open-circuit faults occur in the supercapacitor array [133]. It is more practical to operate the partly degraded supercapacitor array rather than replacing the whole array when the most supercapacitors are healthy. Energy storage array reconfiguration improves dependability, efficiency, and scalability of large-scale battery arrays. The real-time controller proposed in [72, 73] makes decisions and rearranges in series or parallel while bypassing faulty batteries.

\subsubsection{Power Converters}

The energy storage array is not directly connected to the power source or load because of its varying terminal voltage depending on its SoC, 
load current, temperature, and so on. Therefore, a power converter is placed between the energy storage array and power source or load and generates regulated voltage or current for charging or discharging the energy storage array. Batteries and supercapacitors are DC energy storages, and so storing energy from the AC power grid and supplying power from and to the $\mathrm{AC}$ load electronics require $\mathrm{AC}$-to-DC and DCto-AC conversions. An AC-to-DC power converter is often referred as to a rectifier, and a DC-to-AC power converter is often referred as to an inverter.

Power conversion is not free. Converting the voltage level or between $\mathrm{AC}$ and DC involves non-zero amount of power loss. The overall power loss includes conduction losses by parasitic resistances of circuit components, switching losses by parasitic capacitances of switching devices, power consumption of the controller circuit, and so on. The power conversion efficiency $\eta_{c}$ is defined as

$$
\eta_{c}=\frac{P_{\mathrm{out}}}{P_{\mathrm{in}}}=\frac{P_{\mathrm{in}}-P_{c}}{P_{\mathrm{in}}}=\frac{V_{\mathrm{in}} \cdot I_{\mathrm{in}}-P_{c}}{V_{\mathrm{in}} \cdot I_{\mathrm{in}}},
$$

where $P_{\text {in }}$ and $P_{\text {out }}$ is input and output power levels of the power converter, respectively, whereas $P_{c}$ denotes the power loss in the power converter. This power loss is not constant, but varies depending on the input and output voltage and the amount of power that is transferred through the converter, and so the power conversion efficiency also varies. The power conversion efficiency is a critical factor, which determines the energy efficiency of the ESS together with the cycle efficiency of the energy storage elements. Note that the fact that the cycle efficiency of the supercapacitor is nearly $100 \%$ does not guarantee high-energy efficiency of the ESS composed of supercapacitors only because the terminal voltage of such an ESS varies in a very wide range depending on its SoC, which makes it difficult to design an energyefficient power converter. Figure 3.4 shows the efficiency variation of the LTM4609 buck-boost converter from Linear Technology 95]. It shows a wide range of variation depending on input voltage, output voltage, and output current.

Many research efforts have focused on analyzing the power conversion efficiency. Understanding and enhancing the efficiency of power 


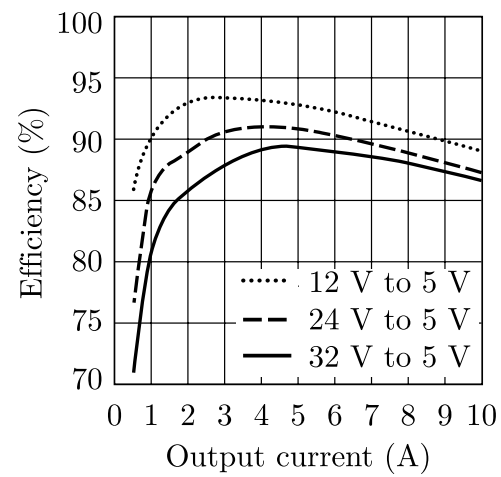

(a) Buck mode

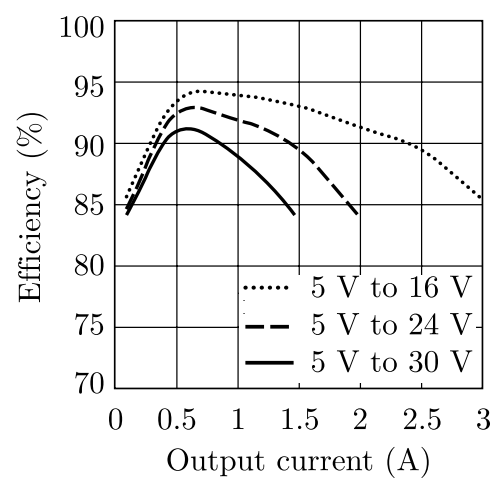

(b) Boost mode

Fig. 3.4 Power conversion efficiency of the Linear Technology LTM4609 buck-boost converter [95].

converters is important for the optimal design and operation of the ESS. The modulation method (PWM vs. PFM) of a power converter has a significant impact on its conduction and switching losses [79. The PWM and PFM DC-to-DC converter efficiencies are studied and specified as functions of the input voltage, output voltage, and output current in 36]. Inverters, which generate AC power out of DC energy storage elements or DC power sources, are also significant. Many different topologies for the power converters are explored in [115, 169].

\subsubsection{System Controller}

Control of the ESS is a not trivial problem. Even a homogeneous ESS requires elaborate system control schemes beyond simple charging and discharging of power converters in order to maximize benefits of the ESS. Objectives of the ESS control typically include enhancing the energy efficiency, cycle life, and reliability.

The basic functionality of the ESS control is management of the energy storage elements. The ESS controller monitors the voltage, current, and temperature of each energy storage element, and prevents the energy storage elements from operating in an unsafe range. Distributed ESS controllers communicate with each other or an external device for monitoring and control through a communication network. Control area 
network (CAN) bus communication is one of the most widely used communication networks for system control [34, 60, 126, 134, which can be used here as well.

An ESS also provides application-specific system controls. ESS controllers for renewable power sources perform MPPT in order to maximize power generation from the power sources [75, 78, 100, 101, 136, 137, 138, 142. The controller is responsible for finding and maintaining the energy-optimal operating point that dynamically varies depending on the environmental conditions. They also should determine the amounts of charge and discharge currents from the power grid considering the power generation and power demand. The optimization objectives include minimizing the daily energy cost considering timeof-use pricing and maximizing battery lifetime [56, 90, 123]. ESS controllers for $\mathrm{EV} / \mathrm{HEV}$ applications determine the direction and amount of power flow among components such as internal combustion engine (ICE), ESS, traction motor, and alternator depending on the operation modes. Optimal system control is critical not only for traction performance, but also for energy efficiency and battery lifetime [52, 111, 116].

System control of HESS is much more complicated than that for a homogeneous ESS. We will discuss this in Section 4.

\subsection{Classification of Energy Storage Systems}

\subsubsection{Homogenous and Hybrid ESS}

\subsubsection{Homogeneous ESS}

Homogeneous ESS are composed of a single type of energy storage elements. Majority of large-scale ESS that are currently deployed are homogeneous. Main advantages of homogeneous ESS is that the homogeneity of energy storage elements gives rise to relatively low design and operation complexities. Selecting the type of energy storage element is most critical for the homogeneous ESS design, whereas other decisions such as provisioning for the required energy and power capacities are naturally determined by performance requirements for the homogeneous ESS and characteristics of the energy storage elements. Batteries are the archetypal energy storage elements, which are most widely 


\section{Energy Storage Systems}

utilized today. New energy storage elements such as supercapacitors are being used in homogeneous ESS targeting some specific applications.

Battery-based ESS There are some high-level control methods developed for lithium-ion battery-based homogeneous ESS for residential purpose [40, 123] to reduce the system capital cost and energy cost by peak shaving and load leveling when time-of-use electricity pricing scheme is applied. Another management method employs dynamic programming and expert knowledge-base rules to reduce the capital cost and/or the energy cost for industrial purpose [87]. ESS for renewable power sources such as PV cells and windmills are utilized for power generation maximization and voltage/frequency regulation. Those applications may be grid-tied [37, 123, 143, or off-gird (standalone) [10, 14, 47, 46]. Capital and energy cost reduction in a systematic way has been an important concern for such renewable power source applications [14, 46, 47]. EV/HEV shown in Figure 3.5 is another application of the battery-based ESS [12, 113]. Research objectives include energy cost reduction and cycle life maximization [12] and energy capacity optimization with power management 113 .

Implementation of an ESS involves many issues including power circuit design and control method design. Some research focus on developing system control methods such as SoC balancing between batteries [28] or power flow control for each battery [104]. System-level design

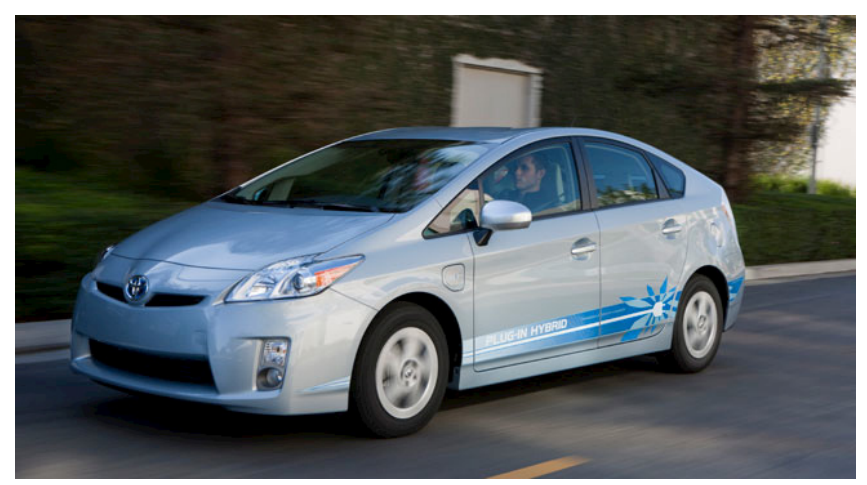

Fig. 3.5 An example of battery-based ESS applications, Toyota Prius PHEV with lithiumion batteries. 
and implementation of a grid-tied ESS prototype using lithium-ion batteries is discussed in [126].

Supercapacitor-based ESS Because of their high-power density, long cycle lives, and high efficiency, supercapacitors are attracting more attention for use in some ESS. Reference [106] introduces and classifies many applications of supercapacitor-based ESS. Energy management/conservation applications take advantage of the high-power capacity and high cycle life of the supercapacitors. Other applications include heavy industrial machineries and heavy vehicles as shown in Figure 3.6(a). Power flow in these applications exhibits frequent charge/discharge cycles of short duration and a large amount of current per cycle, which makes the supercapacitors the energy storage element of choice. An elevator is another such application [132]. The fast-charging feature of supercapacitor-based ESS makes them ideal for home-use power tools such as a cordless screwdriver as shown in Figure 3.6(b).

Supercapacitors in EV/HEV are utilized as a high power and high efficiency energy buffer for acceleration and regenerative braking. A supercapacitor-only EV, which has no other power sources like combustion engine or battery, is introduced in [24]. A voltage equalization method for the supercapacitor HEV is introduced in [168]. However, it is more common to use supercapacitors with other high-energy capacity power sources due to the limitation on the energy density of the supercapacitors [6, 52, 171, 111, 116, 135, 145].

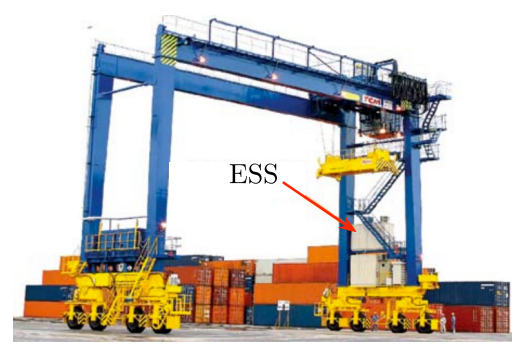

(a) Regenerativenergy storage for a seaport crane

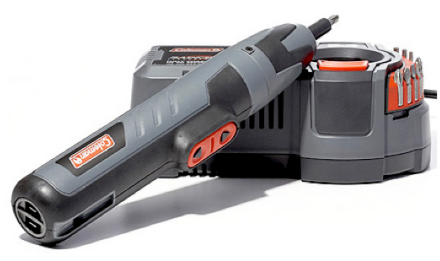

(b) Coleman FlashCell cordless screwdriver

Fig. 3.6 Examples of supercapacitor-based ESS applications [106. 
Even low-power applications such as wireless sensor nodes can take advantage of the supercapacitors. Energy harvesting is common for self-sustainability of the sensor nodes, and supercapacitors serve as a high cycle efficiency energy storage element for the harvested energy in the sensor nodes. Some examples in the literature are solar energy harvesting with MPPT [78, 100, 101, 136, 137, 138, 142] and vibration energy harvesting [146].

Other types of homogeneous ESS We deal with only batteries and supercapacitors in this paper, leaving other types of energy storages out of our scope, but diverse types of energy storages such as kinetic, thermal, and chemical energy storages are also available. Examples include flywheels [5], compressed air [103], hydropower using dams 41], thermal energy storage system [84, and hydrogen-based chemical energy storage system [16]. A white paper from International Electrochemical Commission well summarizes various energy storage systems [2].

\subsubsection{Hybrid ESS}

Homogeneous ESS are inherently subject to the limitations/weaknesses of the energy storage elements that constitute them. For example, battery-based ESS have a limited number of operation years, which is determined by the cycle life of the batteries. Supercapacitor has a very high cost-per-energy, and so it is not very economical to use in low-power high-energy ESS. HESS is an emerging technology that overcomes the limitations of homogeneous ESS by utilizing heterogeneous energy storage elements. For example, the batterysupercapacitor HESS aims at achieving high-energy capacity of the battery and high-power capacity of the supercapacitor at the same time. However, a HESS requires non-trivial initial design and runtime control in comparison with a homogeneous ESS. Not only the types, but also the proportions of each energy storage element, should be carefully determined at design time. Energy flow to each energy storage element during operation is also important. The benefits of the HESS are many and include high-energy efficiency and long lifetime, 
but these benefits are achievable only through careful optimization and management strategies.

Recently HESS has attracted attention from the research community due to its need for systematic optimization and careful charge management at runtime [80, 81, 108, 109, 124. For example, lifetime maximization for a battery-supercapacitor HESS is discussed in [108, 109] with systematic approaches utilized for the EDA problems. There more more examples of power management of power sources and load devices when considering fuel cell-battery hybrid systems [27, 85, 97, 178, 179, 180, 181]. We discuss the HESS in more detail in Section 4 and beyond.

Battery-supercapacitor HESS Battery-supercapacitor hybrid is an exemplary HESS. Research interest in the battery-supercapacitor hybrid is widespread and includes hybridization architecture design, control method, amortized system cost minimization, and so on.

$\mathrm{EV} / \mathrm{HEV}$ are representative applications of the HESS [6, 52, 71, 111, 116, 135, 145. Many research related to HESS design and control in $\mathrm{EV} / \mathrm{HEV}$ focus on improving the energy efficiency of vehicles. A power control method proposed in [111], for example, optimizes the supercapacitor current by using a neural network and achieves more than $20 \%$ improvement in $\mathrm{km} / \mathrm{kWh}$. A power control method introduced in 145, is for a HEV with a shared bus composed of a fuel cell, battery, and supercapacitor. A simple passive parallel connection of battery and supercapacitor mitigates voltage ripple with limited volume and weight of the EV/HEV [52]. An optimization method in [129] solves the multi-objective optimization of minimizing the energy loss and maximizing the power reserve for HEV. Economic viability of using the HESS for EV/HEV is analyzed in [105]. A genetic algorithm-based revenue maximization method for a HESS used in energy and regulation markets is presented in [69].

Low-power sensor nodes like [68, 120] employ a batterysupercapacitor hybrid as shown Figure 3.7. Due to very limited capability to produce power from energy harvesting devices such as PV cells, reducing the power loss during charge/discharge cycles is important. They take advantage of the high cycle efficiency of the 


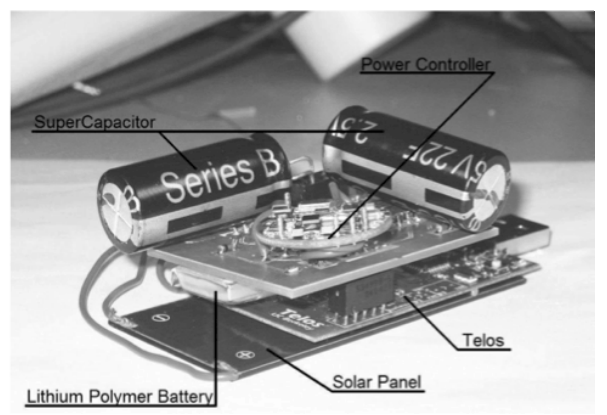

(a) Prometheus

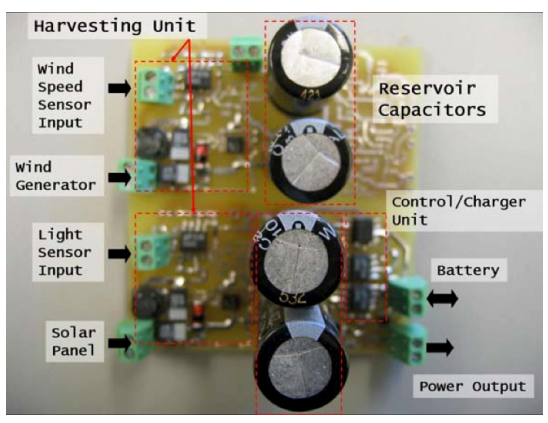

(b) AmbiMax

Fig. 3.7 Battery-supercapacitor hybrid wireless sensor nodes. (a) Prometheus from UC Berkeley [68] and (b) AmbiMax from UC Irvine [120.

supercapacitor while using the battery for low-leakage long-term energy storage.

Other types of HESS A compressed air energy storage (CAES) may utilize supercapacitors to keep track of the maximum efficiency operating point 88]. A HESS introduced in [66] takes advantage of high-power power density of superconducting magnetic energy storage (SMES) and high-energy density of batteries. A fuzzy control logic determines the power split between the SMES and batteries.

\subsubsection{Portable and Grid-scale ESS}

ESS may also be categorized by their scale, which is directly related to the total capacity, portability, and target applications. Small-scale and large-scale ESS have distinctive performance requirements.

\subsubsection{Portable applications}

Portable ESS are used for powering portable electronics, and so have strict constraints on their volume and weight. High energy density per unit volume or unit weight is thus the key criterion for portable ESS. Lithium-ion battery is a most promising type of energy storage element for today's portable applications. Electrical energy is increasingly 
becoming a major concern for today's foot soldiers who must carry more and more advanced power-hungry electronics [9]. Some portable applications such as military radios also require high-power capability with small and light form factor. Battery-supercapacitor HESS may deal with the high-power demand of such applications by using supercapacitors as an energy buffer [139, 140].

$\mathrm{EV} / \mathrm{HEV}$ applications are in between portable small-scale applications and stationary large-scale applications. They can accommodate larger size and weight quota for the HESS, but still require high energy density because it directly affects the cruising distance of the vehicle. Cycle life is another critical factor for EV/HEV applications because they suffer from deep DoD. Low cycle life implies high maintenance cost for battery replacement. Lead-acid batteries have been used for traditional vehicles and EV such as golf carts and forklifts for low cost, but their heavy weight is the problem. Today's HEV such as Toyota Prius has a NiMH battery pack [59. Lithium-ion batteries are being actively researched for use in $\mathrm{EV} / \mathrm{HEV}$ applications to replace the NiMH batteries [12, 113].

\subsubsection{Grid-scale applications}

Large-scale ESS for powering households, factories, or even towns virtually have no restriction with respect to their total volume and/or weight. Battery-based large-scale ESS with tens of MW capacity are already deployed over the world [42]. The types of batteries are also diverse including traditional lead-acid, nickel-cadmium, and lithiumion batteries and emerging flow batteries.

ESS are already being deployed for various purposes of power supply variation compensation and peak load shaving. Reference [42] provides some examples of such homogeneous ESS deployment over the world. These systems are commonly composed of lead-acid batteries, nickelcadmium batteries, lithium-ion batteries, or regenerative fuel cell. A report from the Department of Energy indicates that a battery array incorporated with a PV cell array in a house substantially enhances efficiency, and reduces overall energy cost for the owner despite the capital cost of the battery [1]. 


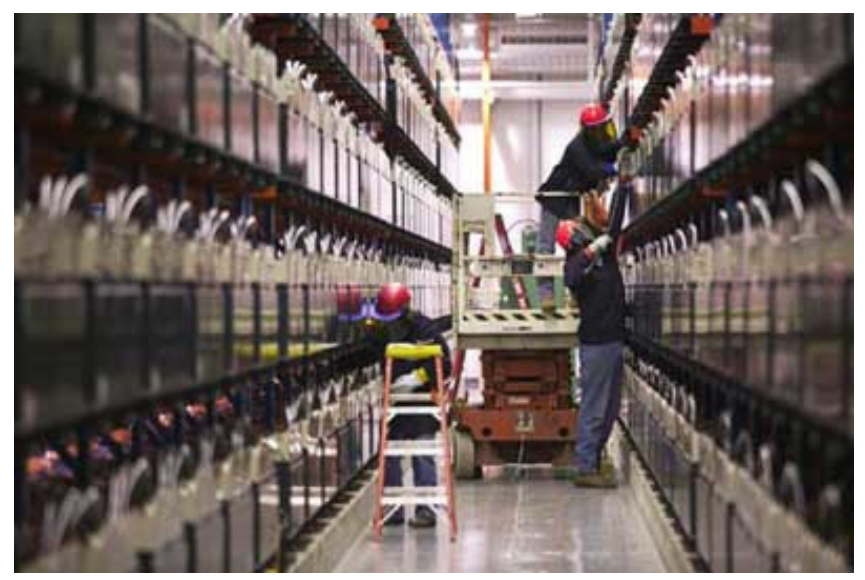

Fig. 3.8 Golden Valley Electric Association, Fairbanks, AK, a 27 MW NiCd battery ESS for the power grid.

Grid-connected ESS serve as a commodity storage for storing energy during off-peak period for use during peak usage period for arbitraging the production price [31, 42, 128]. Such systems can eliminate peak load demand and thereby lower the maximum power generation capacity (peak shaving), or make the load demand uniform over time, which is better from the perspectives of generation, transmission, and distribution systems (load leveling). Another application is contingency service, which supplies power when the grid power generation plants fall off-line. A grid-scale NiCd battery-based ESS shown in Figure 3.8 operated by Golden Valley Electric Association (GVEA) Alaska, is designed to provide 15 minutes of community load against the power failure [128]. Grid-connected ESS also aid the power generation by preventing unplanned transfer of power and maintaining a state of frequency equilibrium. In addition, they may start up on their own and energize the power generation system after a blackout [31].

Stand-alone renewable power sources such as PV cells and windmill generations utilize the ESS for various benefits. More precisely, the ESS for the renewable power sources mitigate mismatch between power demand and uncontrollable power generation due to environmental conditions (for example, solar irradiance, wind strength). Maximizing 
the power generation regardless of variations in the environmental conditions and load demand is crucial for increasing the power generation efficiency of the renewable power sources. ESS are used to decouple the power generation and power consumption to enable MPPT [78, 100, 101, 136, 137, 138, 142]. 


\section{Hybrid Energy Storage Systems}

HESS is an emerging approach for achieving performance improvement of the ESS with current non-ideal energy storage element technologies relying on optimal design and control. Hybridization offers opportunities to take advantage of each energy storage element while hiding their shortcomings/drawbacks. Expected benefits include enhancement of energy efficiency, cycle life, power and energy capacity, and so on. The motivations for the HESS are introduced in Section 4.1 in analogy with the hybridization and hierarchy in computer memory subsystem design. Distinctive characteristics of the HESS compared with ESS in terms of the system architecture and management are discussed in 4.2

\subsection{Motivation and Principle}

The hybrid approach for the HESS has the same motivations as those for designing the memory subsystem in a computer system. Table 4.1 shows characteristics of some representative computer memory devices. SRAM (static random access memory) has the lowest latency and highest throughput but expensive and has low density. On the other hand, DRAM (dynamic random access memory) is inferior to the SRAM in 
Table 4.1. Comparison of density, cost, power consumption (idle/active), 16-bit random access time (read/write/erase) of memory devices [121.

\begin{tabular}{lccccc}
\hline Memory & $\begin{array}{c}\text { Fast } \\
\text { SRAM }\end{array}$ & $\begin{array}{c}\text { Low power } \\
\text { SRAM }\end{array}$ & $\begin{array}{c}\text { Mobile } \\
\text { SDRAM }\end{array}$ & $\begin{array}{c}\text { NOR } \\
\text { flash }\end{array}$ & $\begin{array}{c}\text { NAND } \\
\text { flash }\end{array}$ \\
\hline Density & Low & Low & High & High & Very high \\
Cost $(\$ / G b)$ & 614 & 320 & 48 & 96 & 21 \\
Idle $(\mathrm{mA})$ & 5 & 0.005 & 0.5 & 0.03 & 0.01 \\
Active $(\mathrm{mA})$ & 65 & 3 & 75 & 32 & 10 \\
Read & $10 \mathrm{~ns}$ & $55 \mathrm{~ns}$ & $90 \mathrm{~ns}$ & $200 \mathrm{~ns}$ & $10.1 \mu \mathrm{s}$ \\
Write & $10 \mathrm{~ns}$ & $55 \mathrm{~ns}$ & $90 \mathrm{~ns}$ & $210.5 \mu \mathrm{s}$ & $200.5 \mu \mathrm{s}$ \\
Erase & $10 \mathrm{~ns}$ & $55 \mathrm{~ns}$ & $90 \mathrm{~ns}$ & $1.2 \mathrm{~s}$ & $200.5 \mu \mathrm{s}$ \\
Non-volatile & $\mathrm{No}$ & $\mathrm{No}$ & $\mathrm{No}$ & Yes & Yes \\
\hline
\end{tabular}

terms of latency and throughput, but cheap and has high density. Mass storage devices such as a HDD (hard disk drive) and NOR/NAND flash memory have even lower cost, higher capacity, non-volatility, but are subject to limited random access capability and write count. Composing the required memory space with the SRAM only is infeasible due to its high cost except for supercomputers where cost is not a primary issue. On the other hand, using HDD or flash memory only cannot meet the latency and throughput requirements of the CPU core and suffers from poor random access capability and limited write count. Computer architects, therefore, have remedied this problem by building a hierarchy of different types of memory devices. A typical memory hierarchy example is illustrated in Figure 4.1(a). L1 (level 1) SRAM cache provides the best latency and throughput but the smallest capacity, whereas L2 (level 2) SRAM cache has a bit poor latency and throughput but is larger than the L1 cache. They both generally reside on chip to provide a fast access speed. The DRAM main memory is placed off chip, or sometime on chip for better latency, provides a much larger capacity with a higher latency and lower throughput than the L1/L2 SRAM caches. Finally, the slowest HDD and flash memory are used to provide the largest storage space. There are many policies to utilize this memory hierarchy efficiently, but generally speaking, we use a faster memory to store frequently accessed data and/or code in order to take advantage of its high speed. We overcome the capacity limitation 

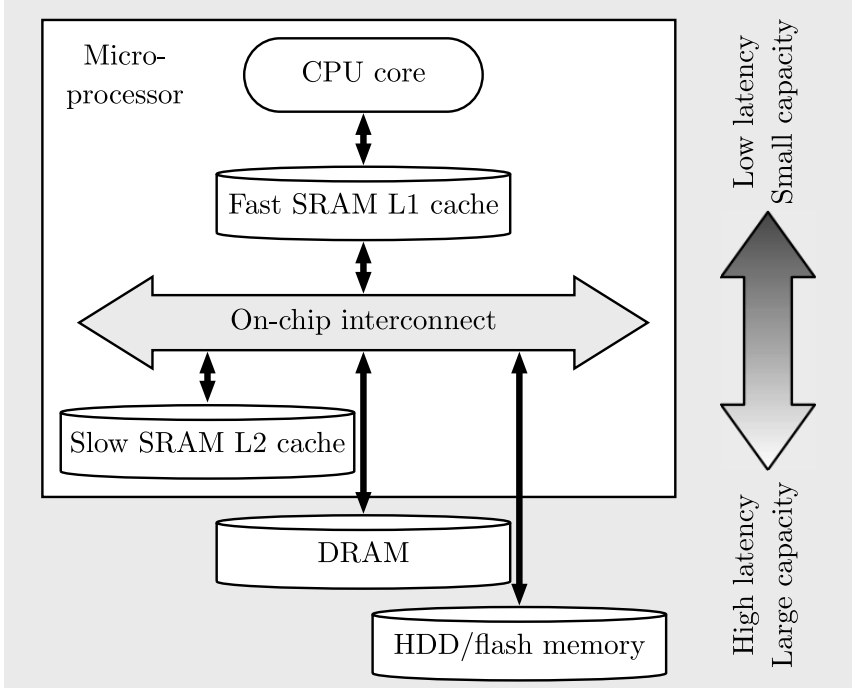

(a) Computer memory hierarchy

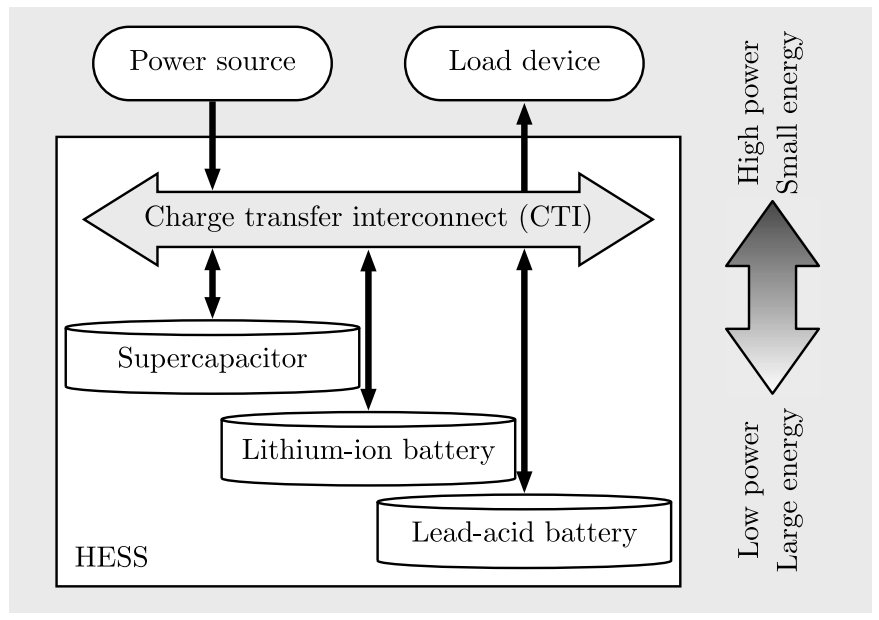

(b) HESS

Fig. 4.1 Comparison of computer memory hierarchy and HESS architecture.

of fast memory by moving less frequently accessed data down to a slower memory. As a result, this memory hierarchy enables the CPU to exploit the low latency of the L1 SRAM cache and the large capacity of the HDD at the same time. 
A HESS aims at similar benefits by using multiple heterogeneous energy storage elements. Instead of relying on a single type of energy storage element, the HESS exploits distinct advantages of multiple heterogeneous energy storage elements and hide their drawbacks. For instance, EV/HEV exhibit frequent charge and discharge cycles with a short period and a large amount of current. Conventional batteries make it difficult to maintain a high efficiency and a longer cycle life in such an operational environment. Use of supercapacitors instead can be a huge upgrade in terms of efficiency and cycle life. However, the current supercapacitor technologies have serious disadvantages in terms of energy density and cost, which makes it hard to completely replace the batteries in an EV with supercapacitors (this is despite a testbed supercapacitor-only EV [24]). Use of supercapacitors in a complementary manner reinforces the drawback of the battery through high-power density, long cycle life, and high efficiency [52, 102, 111, 145].

A conceptual drawing of the HESS is shown in Figure 4.1(b). A HESS is comprised of a number of energy storage banks, and is connected to external power sources and load devices. The HESS in Figure 4.1(b) is comprised of a supercapacitor, a lithium-ion battery, and a lead-acid battery. Similar to the computer memory hierarchy, the HESS exploits different features of these three energy storages to its own benefit: the high-power density and long cycle life of the supercapacitor and the relatively low cost and high-energy density of lithium-ion battery and lead-acid battery. The charge transfer interconnect (CTI) internally connects the energy storages, external power sources, and external load devices though appropriate power converters. In spite of the similarity of the HESS and computer memory subsystem, of course, there are differences as well. For example, there is some energy loss during transfer, but no data is lost during data transfer. Also, selection of storage elements does not cause any coherence or invalidation issues that a cache memory suffers from.

Employing the HESS concept comes with additional design considerations. Deployment of a HESS does not always guarantee better performance unless proper design consideration is given. Designer should thus carefully determine the selection of energy storage elements, the proportion of each energy storage elements, system architecture, the 


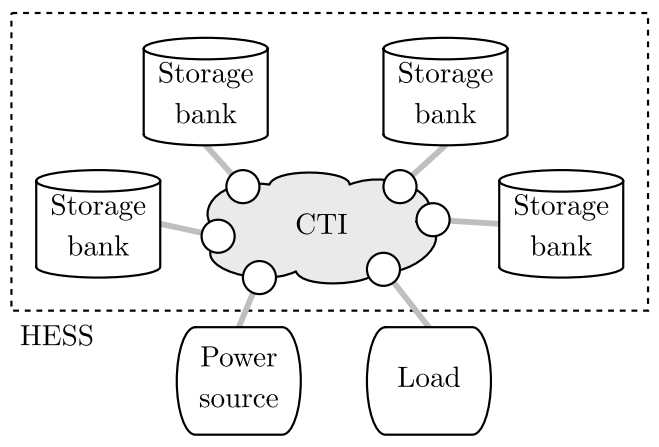

Fig. 4.2 Conceptual diagram of a HESS with four energy storage banks connected through a CTI.

management policy, among others, in order to maximize the benefits of the HESS over the homogeneous ESS.

\subsection{System Architecture}

Figure 4.2 presents a conceptual diagram of a general HESS. There are multiple energy storage banks, which are connected through a network called the CTI. Each energy storage bank is similar to an independent homogeneous ESS, but it performs energy storage array management as determined by system-level HESS management. We discuss the energy storage banks in Section 4.2.1 and the CTI in Section 4.2.2. System control for HESS is discussed in Section 4.2.3.

\subsubsection{Energy Storage Bank}

A HESS is a hybridization of heterogeneous energy storage banks, each of which is homogeneous. An energy storage bank is composed of a homogeneous energy storage array and a power converter as shown in Figure 4.3. which is similar to homogeneous ESS architecture introduced in Section 3.1. In addition to the individual control of energy storage array, an energy storage bank in a HESS supports system-wide bank management. The energy storage banks communicate with a central controller, which sets the system-level management. The controller receives the current status of each bank, such as voltage, current, SoC, 


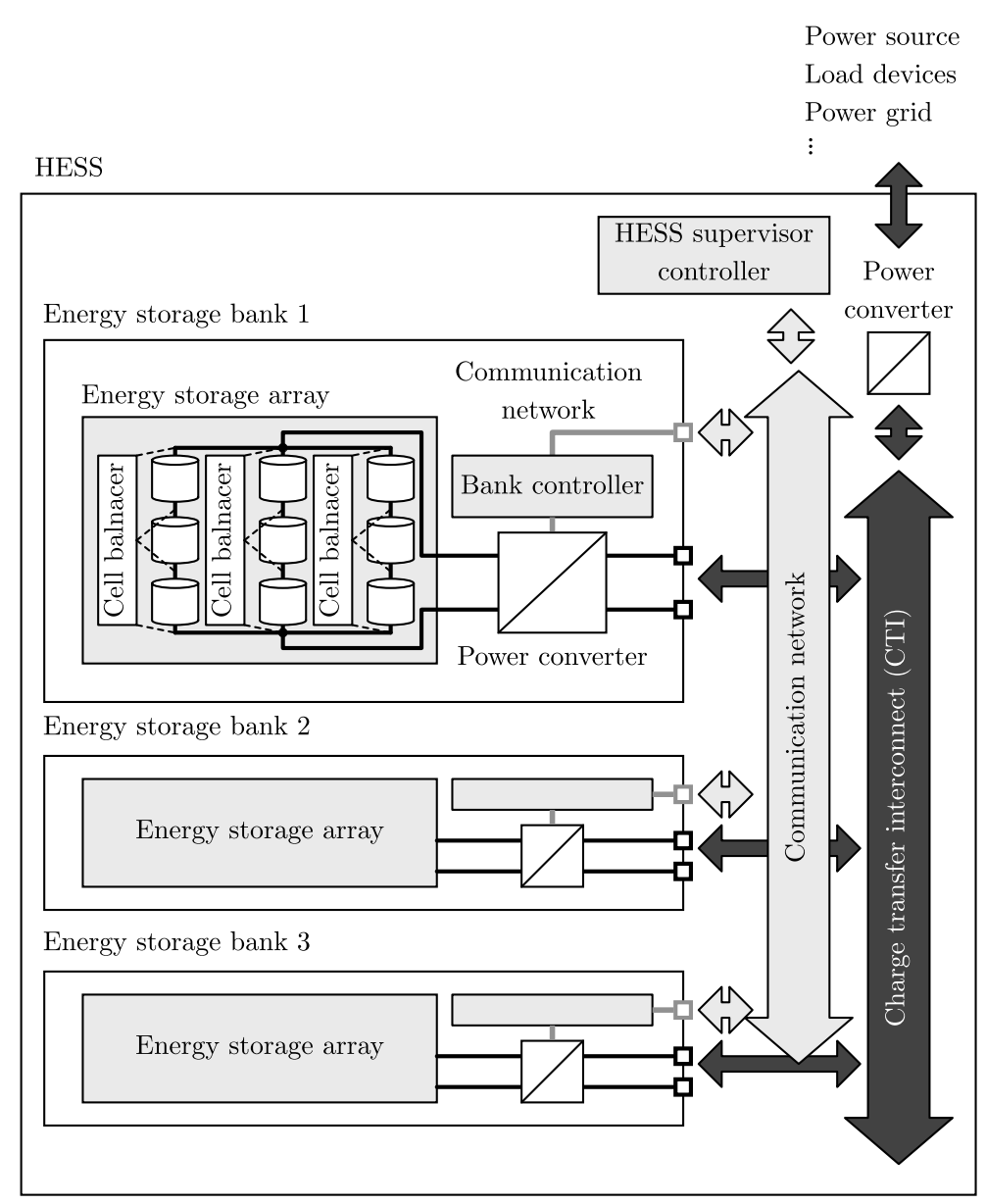

Fig. 4.3 HESS architecture with three energy storage banks.

$\mathrm{SoH}$, and so on, and sends out charge management commands to each bank.

\subsubsection{Charge Transfer Interconnect}

Let us see how the energy storage elements are connected starting from a simple battery-supercapacitor HESS. Figure 4.4 shows three representative hybrid architecture of battery and supercapacitor. A basic 


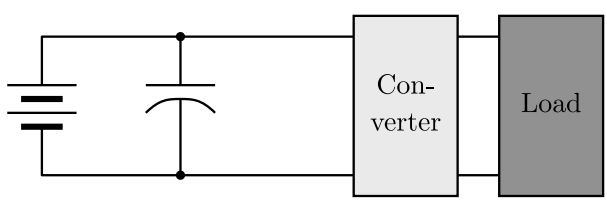

(a) Passive parallel connection

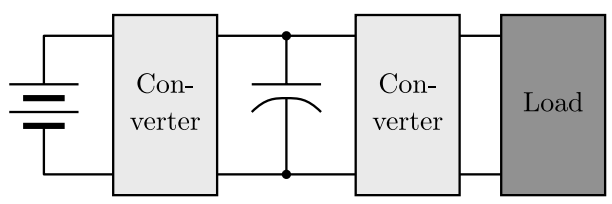

(b) Cascaded converters

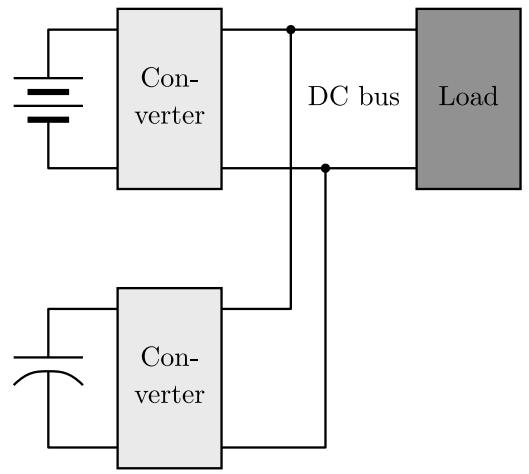

(c) DC bus topology

Fig. 4.4 Supercapacitor-battery hybrid connection topologies.

hybrid approach is a passive direct connection of a battery and a supercapacitor in parallel [44] as shown in Figure 4.4(a). The supercapacitor handles high current demands by suppressing large battery voltage variations, but this architecture cannot be generally applied for different combinations of heterogeneous energy storage elements. Cascaded converter architecture shown in Figure 4.4(b) puts a converter in between the battery and supercapacitor to control the power. A constant-current regulator-based hybrid architecture for a battery and supercapacitor in [139, 140] improves the energy efficiency compared with the direct parallel connection. The parallel connection and cascaded converters architecture, however, are not suitable for three or more energy storage banks.

A general hybrid architecture, which can accommodate any type of energy storage elements in a symmetric manner, utilizes a DC bus. In the DC bus architecture, each energy storage element is connected to the DC bus through a power converter (voltage regulator or current regulator). A HESS introduced in [145] is comprised of a fuel cell, a battery, and a supercapacitor connected to a fixed-voltage DC bus as shown Figure 4.4(c). The control method regulates a fast energy storage element with a slow energy storage element in order; it controls the DC bus voltage, supercapacitor voltage, and battery voltage by controlling the supercapacitor current, battery current, and fuel cell current, 
respectively. Another HESS is comprised of a photovoltaic panel, a fuel cell, and a supercapacitor, and its basic operational principle is the same 144. This simple and intuitive control method, however, cannot achieve energy optimality because (i) the DC bus voltage is fixed, and so the conversion efficiency is not always the maximum; and (ii) current distribution among the energy storage elements is determined by other energy storage elements, and cannot be optimized in a holistic manner. True energy optimality requires consideration of not only the dynamic response, but also other factors such as rate capability, residual energy, and load demand. For example, we may want to reserve energy in the supercapacitor and use the battery instead when a high current demand is expected in a near future, but this kind of intelligent energy control is not supported in [144, 145]. Another DC bus-based HESS proposes a control method that increases the supercapacitor current as the battery current increases [174].

Heterogeneous energy storage elements in a HESS plays distinct roles depending on their unique characteristics, but may or may not have an hierarchy among the elements. A hierarchy may be physical (explicit hierarchy that is exposed by the CTI architecture) or logical (implicit hierarchy achieved by system management policies). For example, the cascaded converters architecture has an explicit hierarchy, whereas the parallel connection and DC bus architecture do not. However, the DC bus architecture may have logical hierarchy between the battery and supercapacitor by actively controlling their contribution in various aspects such as power and energy.

A HESS with larger number of energy storage banks requires a more complicated connection than a passive parallel connection or cascaded converter architecture as the CTI. Connecting heterogeneous energy storage elements in a HESS is more than expanding power and energy capacities, but involves complicated energy transfer among the elements. Therefore, the CTI architecture is more critical in a HESS than in a homogeneous ESS though the CTI architectures for the HESS can be used for homogeneous ESS as well. Similar to on-chip communication networks, the network topology of the CTI is one of the important design considerations for energy efficiency in a large-scale HESS. It has a significant impact on the charge transfer efficiency, and thus should 
be carefully designed in order to maximize the benefits of the HESS. A system-on-chip design is subject to a similar problem of determining a suitable interconnect architecture. The interconnect architecture in a system-on-chip affects its communication latency, throughput, power consumption, and so on. For both HESS and system-on-chip designs, the interconnect architecture should be selected by considering the scale of the system. The interconnect architecture becomes more critical as the number of nodes increases.

CTI architectures of HESS are similar to the system-on-chip interconnect architectures. Figure 4.5 shows four interconnect architectures for four nodes. Figure 4.5(a) is a shared bus interconnect. It is simple to implement, but has a limited scalability. Variances of the shared bus CTI with higher scalability include a segmented bus CTI in Figure 4.5(b) and multiple bus CTI in Figure 4.5(c). The point-topoint interconnect in Figure 4.5(d) provides independent paths between every pair of nodes, but its cost increases exponentially as the number

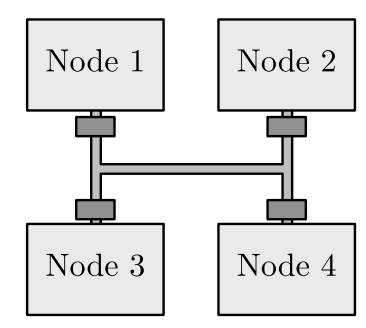

(a) Shared bus

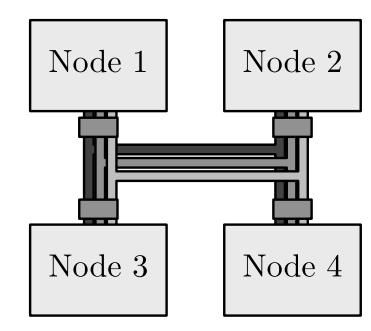

(c)Multiple bus

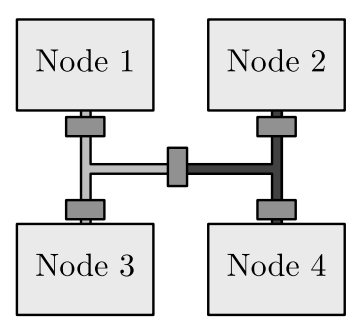

(b) Segmented bus

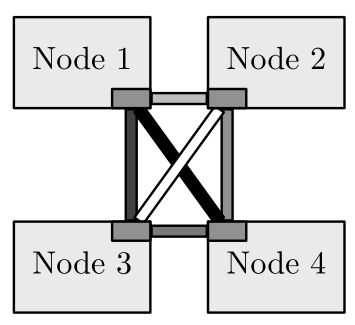

(d) Point-to-point

Fig. 4.5 Various interconnect architectures for system-on-chip and HESS. 


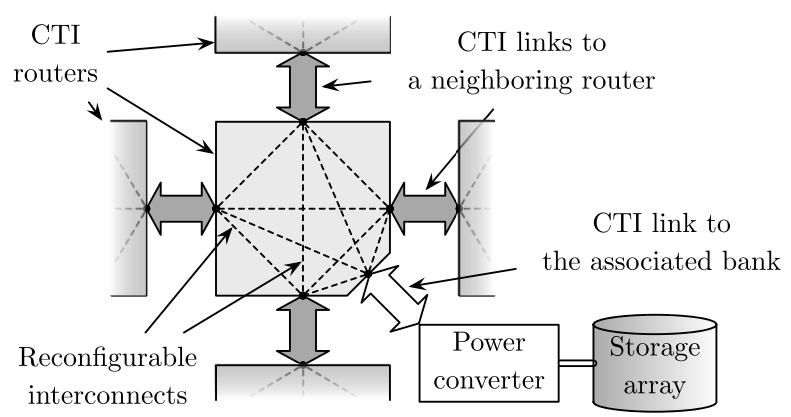

Fig. 4.6 Architecture of a CTI router. An associated energy storage bank is connected via a power converter. The arrows denote the CTI links.

of nodes increases. These architectures are well explored for the systemon-chips, and also applied for the HESS [154, 157, 164, 165, 166, 167.

As the number of energy storage banks in HESS further increases, the CTI architectures in Figure 4.5 cannot provide efficient paths for charge transfers. Higher energy efficiency may be achieved if the CTI network is able to provide more isolated paths to simultaneous charge transfers for the energy-optimal CTI voltage. A networked CTI architecture proposed in Figure 4.6 is comparable to a typical networkon-chip architecture [76. As the number of processing elements in a network-on-chip increases, the single-level on-chip bus architecture is no longer able to handle increased data exchanges between the processing elements. Similar to the network-on-chip that requires packet routing, a HESS with a networked CTI architecture requires routing of the charge transfers.

\subsubsection{System Control}

The HESS requires more sophisticated management policies than a conventional ESS because of the heterogeneity of energy storage elements. Proper management policies are very crucial to achieving high-energy efficiency in the HESS. The HESS management policies are system-level policies for maximizing the benefits of the HESS in energy efficiency, lifetime, etc, by exploiting its heterogeneity.

The most important decision required in a HESS is energy distribution among heterogeneous energy storage banks. We need to select 
particular energy storage banks to charge or discharge and determine the CTI voltage and amount of current that maximize the energy efficiency. Also, we may need to internally move energy from one energy storage bank to another in order to mitigate the self-discharge or prepare for the expected demand for energy/power capacity. These operations are called charge management, which is named after cache management in computer memory hierarchy in [124]. The charge management includes (i) charge allocation for charging energy storage banks 164, 165], (ii) charge replacement for discharging energy storage banks [166], and (iii) charge migration for moving energy between the energy storage banks [154, 157, 167]. A SoH-aware charge management enhances battery lifespan by utilizing supercapacitors for high frequency power 163. We discuss system control issues and optimization methods in more detail in Section 5 . 


\section{Hybrid Energy Storage System Design and Optimization}

\subsection{Design-time Optimization}

The total energy capacity is one of the most important features in the HESS specification. However, HESS requires determination of the type of storage banks and individual storage bank capacities at the design time. The analogous problem in computer memory system design is that of determining the capacities of L1, L2, and L3 caches, the main memory, and the disk. Determining the individual energy storage bank capacity is more similar to partitioning the on-chip scratchpad memory in that the physical structure is flat, and there is no inclusion properly requirement for each level of the logical hierarchy. Appropriately-proportioned energy storage bank capacities can enhance HESS performance metrics, whereas smart control policies can create the illusion that the HESS consists of only the most suitable energy storage elements for the given load. Determination of the energy capacity of each energy storage bank in the HESS is a critical issue for minimizing the cost of the HESS. Not only the capital cost for purchase and disposal of the energy storage elements, but also the operational cost for electricity energy is affected by the composition of energy storage bank types. 
Example bank energy capacity design Let us consider a HESS that consists of a lithium-ion battery bank and a supercapacitor bank. The two energy storage elements have distinct characteristics, that is, lithium-ion batteries have an $80 \%$ round-trip efficiency, 1,000 $\$ / \mathrm{kWh}$ dollar cost, $0.1 \%$ self-discharge rate, and a cycle life of 2,000 , whereas supercapacitors have a nearly $100 \%$ round-trip efficiency, 40,000 $\$ / \mathrm{kWh}$ dollar cost, $15 \%$ self-discharge rate, and 100,000 cycle life. Suppose we are required to compensate energy usage fluctuation. We have a small daily fluctuation and a large weekly fluctuation, and the total fluctuation is a superposition of them. A homogeneous ESS composed of only batteries will have a poor round-trip efficiency for daily storage and retrieval of energy. On the other hand, supercapacitor-only ESS will suffer from high leakage for the energy stored during weekend. By building a HESS with optimized composition of the energy storage elements, we can reduce both the capital cost and operational cost. Supercapacitors have near perfect round-trip efficiency and a long cycle life, so they are suitable for compensating daily fluctuation. On the other hand, batteries, which have low round-trip efficiency and a short cycle life, are suitable for weekly energy storage. For an example of 500 $\mathrm{kWh}$ HESS, we have shown up to $15 \%$ cost reduction compared with homogeneous ESS [26].

Another important feature of the HESS specification is its overall power capacity, which in turns depends on the power capacity of each energy storage bank in the system, power capacity of the charger and the DC-DC converter, and the control policies employed. The power capacity of each energy storage bank in the HESS is analogous to the access speed of the memory array, the charger and the DC-DC converter power capacity may be compared to the clock frequency of the interconnection network, whereas the HESS control policies are analogous to the caching policies in the computer memory system. We define the power capacity optimization problem as that of designing a HESS that satisfies a power capacity requirement while maximizing the total service time.

Example bank power capacity design Suppose we have a high current pulsed load device such as a radio transceiver. Consider a (series 
connected) 2-cell lithium-ion battery of $350 \mathrm{mAh}$ capacity used along with a supercapacitor in a HESS. The load device consumes $3.5 \mathrm{~A}$ of current for 10 seconds with a $10 \%$ duty ratio. We can enhance the total service time of the battery-supercapacitor HESS by use of a constantcurrent charger circuit compared with a conventional hybrid architecture that simply connects the battery and supercapacitor in parallel. An optimization method introduced in [139] derives that a supercapacitor of $2.5 \mathrm{~F}$ capacity maximizes the total service time of the HESS. Experimental results of a real implementation of the constant-current charger-based HESS show 7.7\% total service time improvement.

Design optimization of a residential HESS In [177], we investigated the design and control optimization of a HESS for a residential smart grid user in order to maximize the total profit. We consider a HESS comprised of a lithium-ion battery bank and a lead-acid battery bank for a case study. We consider a simple time-of-day electricity pricing function [38] as shown in Figure 5.1] Both energy storage banks will get charged during the low peak period and discharged during the high peak period under such electricity pricing function so as to reduce the daily electricity cost for the residential user. We take into account the following factors in the design optimization framework to provide more practical and accurate estimation of the amortized annual profit:

- Characteristics of different battery banks, including energy capacity, power capacity, rate capacity effect, self-discharge rate, and so on.

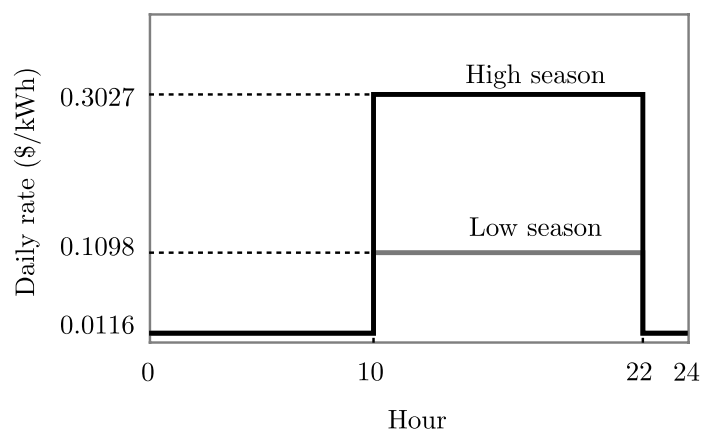

Fig. 5.1 Daily time-of-day energy pricing [38]. 
- Power conversion efficiency variation of chargers and power converters.

- The SoH degradation rate, or the cycle life, of an energy storage bank is strongly dependent on the DoD. Reference work [107, 163] shows that the battery cycle life increases (nearly) exponentially when the DoD decreases.

We determine the energy capacity of both energy storage banks subject to the following constraints.

- System volume constraint: we must limit the overall volume of the HESS since it targets residential usage, so

$$
\begin{aligned}
\text { Volume of Li-ion bank } & + \text { Volume of lead-acid bank } \\
& \leq \text { Total volume constraint }
\end{aligned}
$$

Our problem formulation uses the reciprocal of battery volumetric energy density, referred to as the unit volume, which is its volume divided by the maximum stored energy. The lead-acid battery's average unit volume is $12.5 \mathrm{~L} / \mathrm{kWh}$, much higher than that of lithium-ion battery: $2 \mathrm{~L} / \mathrm{kWh}$ [124].

- Monetary budget constraint: The total cost of the HESS consists of both the capital cost of energy storage banks and the maintenance cost. We know that lithium-ion batteries usually have 3-4 times the life time of lead-acid batteries. Normally different types of batteries do not reach their endof-life together. It is uneconomical to discard or replace the whole HESS as soon as one battery bank reaches its end-oflife. Instead, replacing the aged battery bank with a new one can restart the HESS with lower extra cost. Apart from the purchase cost of new batteries, the replacement of devices also adds up to the total cost since it requires maintenance personnel to come in and restore the system. Moreover, we also consider the discount factor, which signifies the fact that there is a difference between the future value of a payment and the present value of the same payment. The overall 
monetary budget constraint is given by:

$$
\begin{aligned}
& \text { Amortized cost of Li-ion bank } \\
& \quad+\text { Amortized cost of lead-acid bank } \\
& \quad+\text { Amortized maintenance cost } \\
& \leq \text { Monetary budget constraint }
\end{aligned}
$$

where the discount factor is reflected in the amortized costs of different parts.

We have proposed a unified framework for the optimal design and control of the HESS targeting at exploiting its potential for energy cost saving. First, we derive an effective HESS control algorithm to maximize the daily energy cost saving with a given specification of the HESS (in terms of types and capacities of different energy storage banks), under limitation on the DoD. This management algorithm properly controls the charging and discharging of each energy storage bank. For each possible design specification of the HESS under a monetary budget constraint and a total volume constraint, we can find its amortized annual profit based on the optimal control algorithm. We find the optimal design and specification of the HESS that maximizes the amortized annual profit. We show that the optimally designed HESS achieves an annual return on investment (ROI) of up to $60 \%$ higher than a leadacid battery-only system or a lithium-ion battery-only system, under the same amount of investment. We provide the comparison results on the annual profit in Figure 5.2 .

Future work on the design optimization of HESS may include (but is not limited to) the following directions.

- Perform joint design optimization on both the PV module and the HESS in order to achieve the maximum amortized profit, under a given budget of the total investment.

- Study parameters for future energy storage technologies, such as their energy density, unit capital cost, and so on. In this way, we may explore the potential benefits of HESS in the future. 


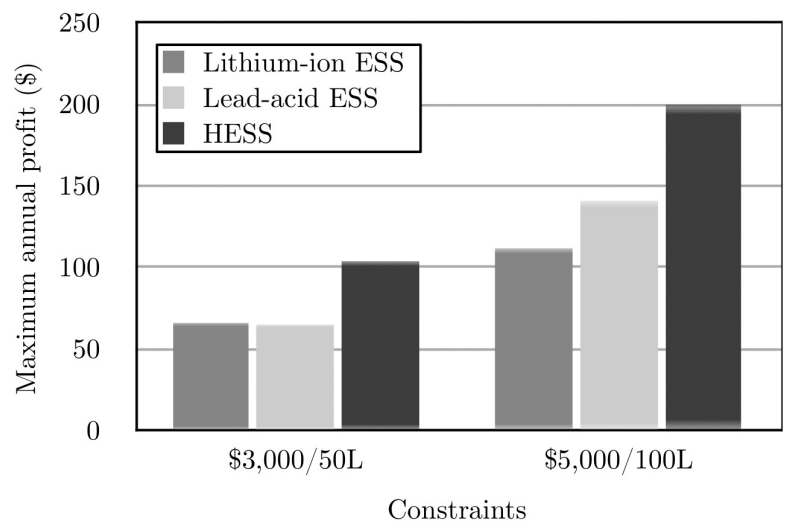

Fig. 5.2 Maximum annual profit with different constraints [177.

\subsection{Runtime Optimization}

\subsubsection{Charge Management}

An operative framework for designing a HESS should enable holistic optimizations across different HESS components and structures through unified problem formulations and efficient solutions. Simultaneously, this framework should enable multi-objective optimizations with respect to cycle efficiency, cycle life, system cost, and so on. For illustration purposes and without loss of generality, this article focuses on cycle efficiency enhancement.

The cycle efficiency of an ESS is defined as the ratio between the energy deposited and retrieved. The ratio is always less than one because of the energy loss during charging and discharging. Mainly the energy storage arrays and the power converters cause energy loss. IR loss of the CTI and switch elements also causes power loss, but the loss is not dominant. The type of energy storage array such as supercapacitors, lithium-ion batteries, lead-acid batteries, and so on, largely determines the energy loss for charging and discharging operations by the internal resistance and rate capacity effect [93, 124]. However, the energy loss is also heavily dependent on the operating conditions such as the magnitude of current, SoC, and temperature. The type of converter primarily determines the power loss in the power converters such as synchronous, buck, boost, and flyback. At the same time, the 
operating conditions greatly affect the power conversion efficiency such as input voltage, output voltage, and current. The system efficiency is changing during the charge and discharge operation continuously. Therefore, maintaining the highest possible efficiency needs continuous updating of the charging and discharging current, which is not a trivial problem.

It is crucial to provide policies and methods for charge allocation to the most suitable energy storage banks for a given incoming power level, and for charge replacement from the most suitable energy storage banks for a given load demand. Hence, the HESS run-time control comprises of policies that dynamically control the manner in which the system is used once it has been deployed. These policies include algorithmic/heuristic approaches for basic energy management operations like charge allocation, charge replacement, and charge migration 124. These also include heuristics that predict the future energy needs [165, heuristics that predictively charge some energy storage banks [155], and so on. These operations are illustrated in Figure 5.3. Even if the optimal charge allocation and replacement policies are put in place and executed, charge migration that moves charge from one energy storage bank to another is often necessary to improve the overall HESS efficiency and responsiveness. Charge migration can ensure the availability of the most suitable energy storage bank (in terms of its self-leakage, output power rating) to service a load demand (to the extend possible.) To achieve this goal, we must first invent an efficient low-cost charge migration architecture. We must also develop systematic solutions for multiple source and multiple destination charge migration considering the efficiency of chargers, rate capacity effect of the storage element, terminal voltage variation of the storage element as a function of the SoC, and so on.

\subsubsection{Charge allocation}

Charge allocation determines (i) destination energy storage banks to be charged from an external power source, (ii) the amounts of charging current of destination banks, and (iii) the CTI voltage. The goal of the charge allocation problem is to maximize the system's charge allocation 


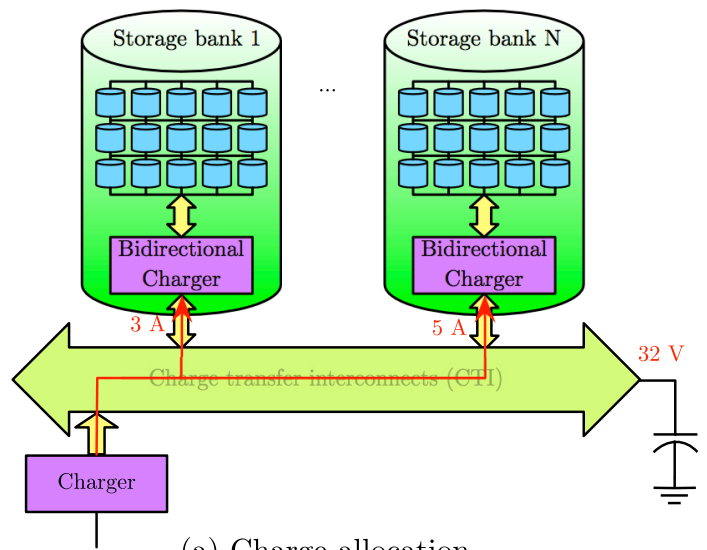

(a) Charge allocation

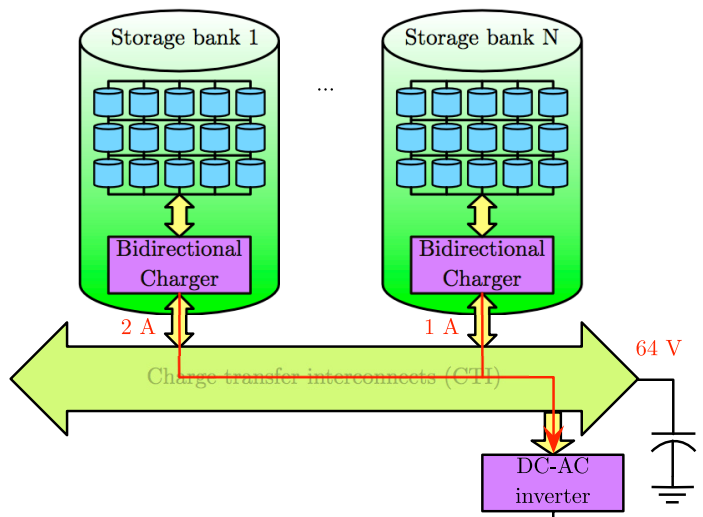

(b) Charge replacement $\left.\right|_{\text {AC load }}$

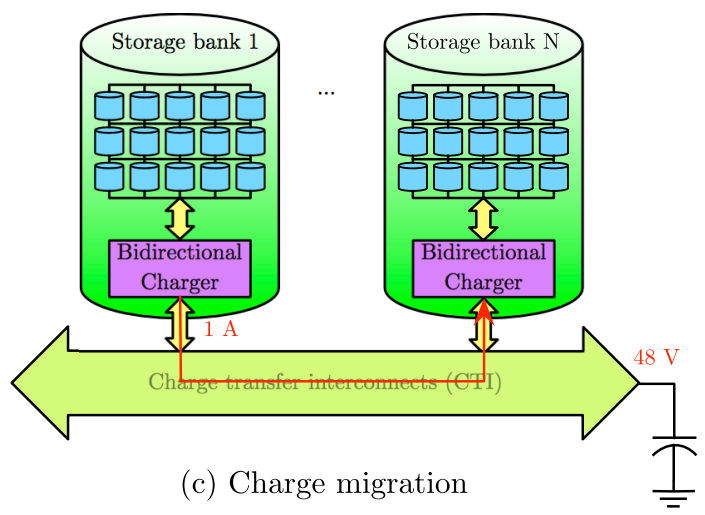

Fig. 5.3 Conceptual diagram of charge allocation, replacement and migration. 
efficiency (also known as charging efficiency), which is given by:

Charge allocation efficiency

$$
=\frac{\text { Total energy gained in all energy storage banks }}{\text { Total energy extracted from the power source }} \text {. }
$$

As HESS comprises of multiple heterogeneous energy storage banks unlike conventional homogeneous ESS, selection of the most efficient energy storage banks to be charged is the key to achieving high charge allocation efficiency [164, 165. Charging efficiency is strongly dependent on the type of the energy storage banks, the magnitudes of the charging currents, SoCs of the energy storage banks, voltage and current characteristics of the external power source, and so on. Stark mismatch between the input voltage level and the energy storage bank terminal voltage results in unnecessarily large power loss in the chargers. Severe mismatch between the input power level and the destination energy storage bank power capacity results in a high power loss due to IR loss and rate capacity effect. The destination energy storage banks must be compatible with the input power source in terms of the energy capacity as well.

A greedy charge allocation policy may choose the destination energy storage banks only based on their instantaneous charging efficiency. For example, such a policy tries to charge mainly energy storage banks with a high-power capacity such as a supercapacitor bank. Supercapacitor banks generally have a low energy capacity and will be fully charged soon at an early stage of the charge allocation process. During the rest of the charge allocation process only battery banks with lower power capacity can be charged, which results in a low overall charging efficiency for the whole charging process. The CTI voltage significantly affects the efficiency of the chargers, and thus it should be carefully determined by the charge allocation control algorithm. The optimal charging currents and the CTI voltage change over time as charge allocation progresses. We continue to monitor the system status, calculate the optimal setup and control the charge allocation process accordingly.

Dividing the whole charge allocation period into a fixed number of timing intervals can significantly reduce the problem complexity [164, 165]. The boundary point between two consecutive intervals is 


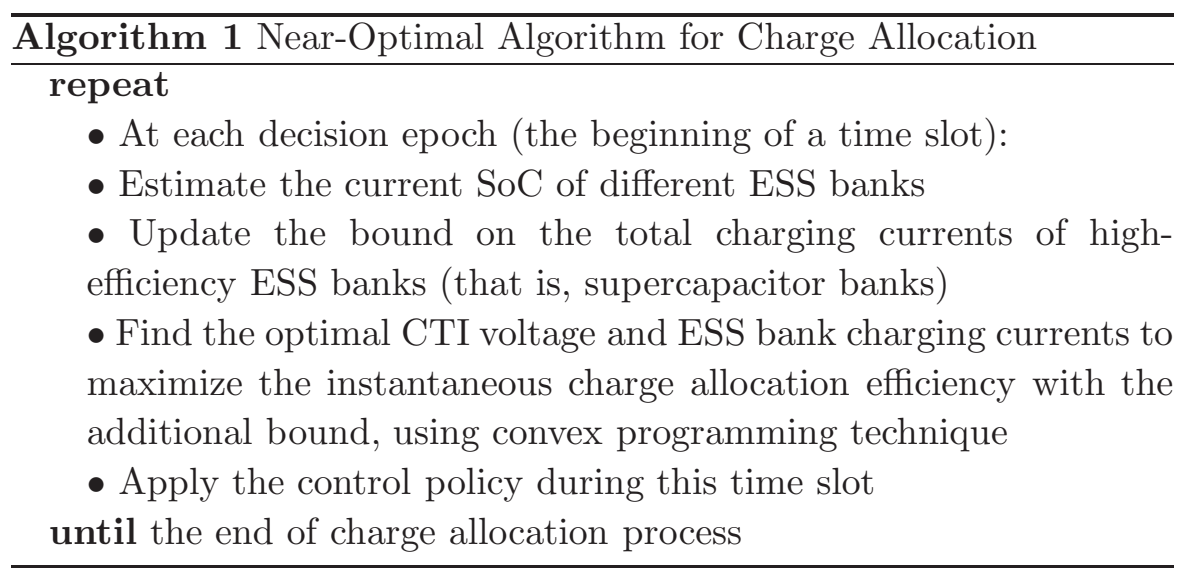

called a decision epoch. We solve an instantaneous charge allocation (ICA) optimization problem to maximize the instantaneous charge allocation efficiency by a convex programming approach at each decision epoch [17. We avoid fully charging of high-power capacity energy storage banks at an early stage of the charge allocation process with additional constraints on the charging currents, thereby maximizing the overall charging efficiency. A brief procedure of the near-optimal charge allocation algorithm is in Algorithm 1. In [165], we further integrate the near-optimal charge allocation algorithm with solar power generation prediction and maximum power transfer tracking (MPTT) technique for the PV panels [78].

The energy storage bank reconfiguration techniques proposed in [77] can be effectively integrated with the proposed charge allocation algorithm. We find the optimal energy storage bank configuration (series-parallel connection) to provide a better match between the energy storage bank terminal voltage and the CTI voltage. We provide further improvement on the charge allocation efficiency in this way through reducing power dissipation in the power converters [36]. Similar methods also apply for the charge replacement and charge migration optimizations.

Figure 5.4 shows the instantaneous charging efficiency, battery bank charging current, and supercapacitor bank charging current. The instantaneous charge allocation efficiency of the proposed algorithm is 

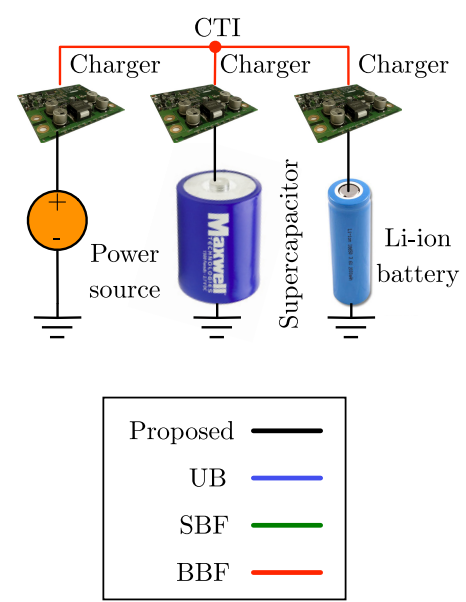
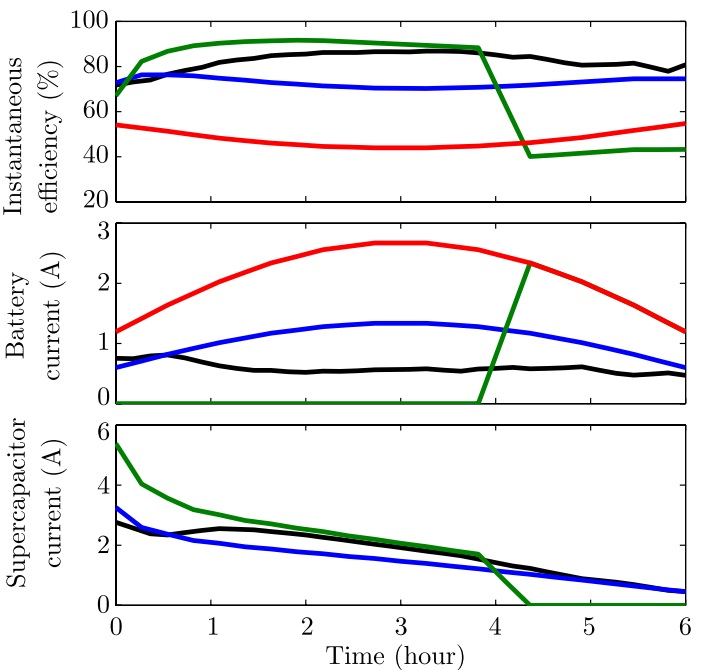

Fig. 5.4 Comparison of charge allocation efficiency among different polices. UB: unbiased charging (equal charging current for both banks). SBF: supercapacitor bank first (charging the supercapacitor bank first). BBF: battery bank first (charging the battery bank first).

always higher than the UB or BBF policies. Although the initial instantaneous charging efficiency of the proposed algorithm is slightly lower than the SBF baseline, the efficiency of SBF drops significantly after the supercapacitor bank becomes fully charged. The improvement of charge allocation efficiency by using the proposed near-optimal algorithm ranges from $8.6 \%$ to $35.6 \%$ compared with baseline setups.

\subsubsection{Charge replacement}

Charge replacement selects one or more source energy storage banks and also determines the amounts of discharging current of the source energy storage banks for a given load. The goal of the charge replacement problem is to maximize the charge replacement efficiency (also known as discharging efficiency), given by:

Charge replacement efficiency

$$
=\frac{\text { Total energy required by the load devices }}{\text { Total energy extracted from the energy storage banks }} \text {. }
$$




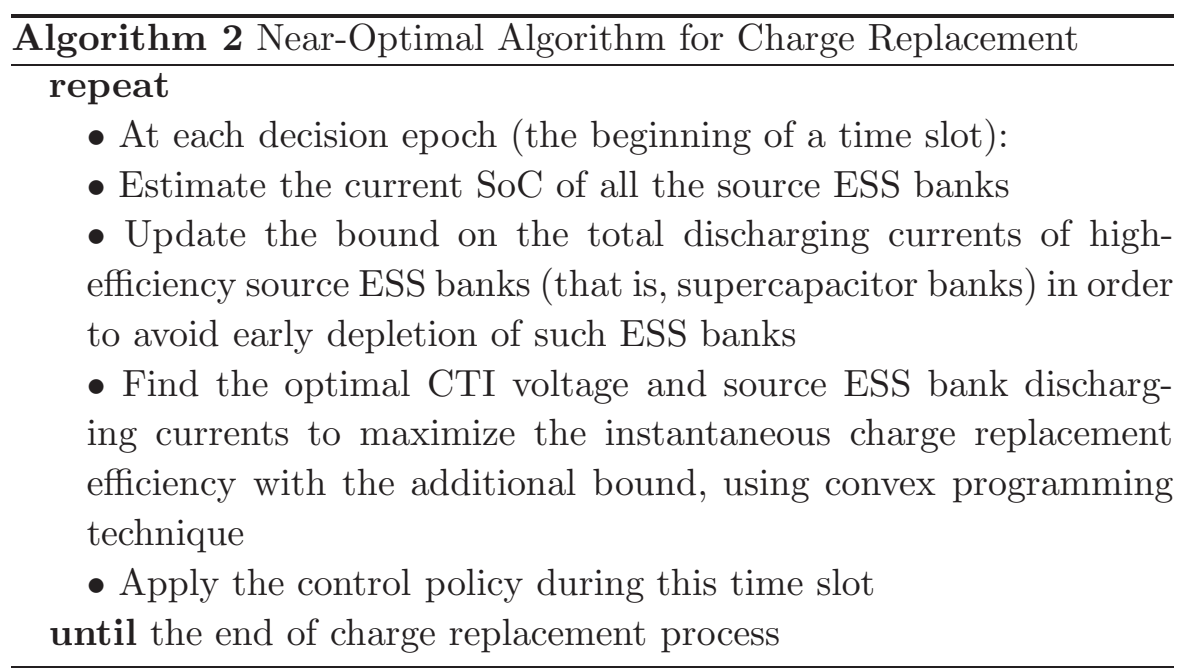

Discharging efficiency is strongly dependent on the type of selected energy storage banks and the magnitudes of the discharging currents, $\mathrm{SoC}$ of the source energy storage banks, and the load voltage and current characteristics. Therefore, compatibilities in terminal voltage, power capacity and energy capacity are again key factors in selecting the source energy storage banks and determining their discharging currents. The best-suited set of source energy storage banks changes over time, and we recalculate the source bank selection, discharging current, and CTI voltage setting at each decision epoch [166]. A brief procedure of the near-optimal charge replacement algorithm is shown in Algorithm 2, which is similar to the charge allocation algorithm.

As shown in Figure 5.5, the instantaneous charge replacement efficiency of the proposed algorithm is always higher than the UB or BBF policies. The instantaneous charge replacement efficiency of the SBF policy again drops significantly after the supercapacitor bank gets fully discharged. The improvement of charge replacement efficiency by using the proposed near-optimal algorithm ranges up to $24 \%$ compared with baseline setups.

\subsubsection{Charge migration}

Charge migration exchanges charge among various energy storage banks. The optimal energy storage banks for charge allocation and for 

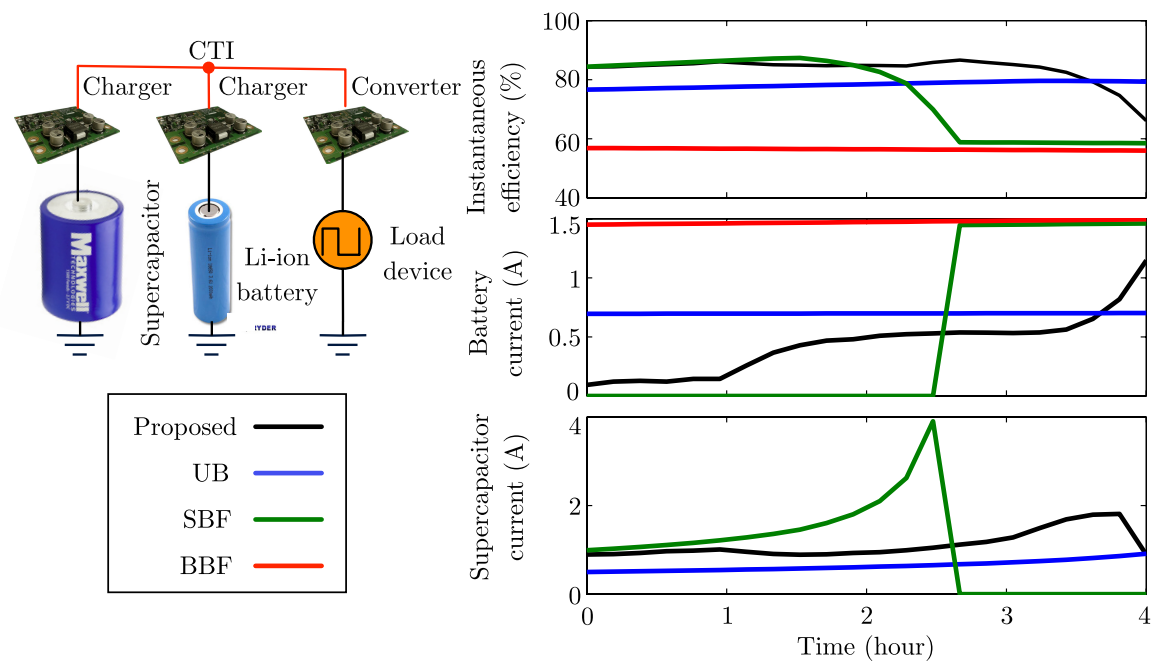

Fig. 5.5 Comparison of charge replacement efficiency among different polices. UB: unbiased discharging (equal discharging current for both banks). SBF: supercapacitor bank first (discharging the supercapacitor bank first). BBF: battery bank first (discharging the battery bank first).

charge replacement can be different in general. Some energy storage banks, such as a supercapacitor bank, are not suitable for long-term energy storage due to large self-discharge rate despite their high efficiency for charge allocation and/or charge replacement [124, 154]. This motivates charge migration. Charge migration maximizes the power compatibility of energy storage banks. For instance, even a supercapacitor bank that is compatible with an incoming power source may be no longer compatible in the near future as it becomes fully charged. However, charge migration is an expensive process due to energy loss. It is additional overhead that is not necessary in a homogenous ESS. It should thus be performed judiciously based on accurate prediction of power profiles of the power source and load devices.

We formulate the charge migration optimization problem such that a given amount of energy from a set of source energy storage banks is transferred to a set of destination energy storage banks within a predefined deadline time. We minimize the amount of energy drawn from source energy storage banks. The goal of the charge migration optimization problem is to maximize the charge migration efficiency, 
given by:

Charge migration efficiency

$=\frac{\text { Total energy transferred into the destination energy storage banks }}{\text { Total energy extracted from the source energy storage banks }}$.

A simple case of charge migration optimization problem is the single-source, single-destination (SSSD) charge migration [154]. We drive the optimal solution of the SSSD charge migration problem without deadline constraint (also known as time-unconstrained charge migration) by maximizing the instantaneous charge migration efficiency at each decision epoch using a branch and bound algorithm or a ternary search algorithm. We derive a near-optimal solution of the SSSD charge migration problem with a deadline constraint (also known as time-constrained charge migration) [154]. Lookup table and high-order curve fitting techniques are incorporated in the charge migration controller to reduce the online computational overhead. We provide a systematic solution for a more general multiple-source, multiple-destination (MSMD) charge migration problem [157] as shown in Algorithm 3. The instantaneous MSMD charge migration optimization problem at each decision epoch is solved using an efficient quasi-convex programming approach [17].

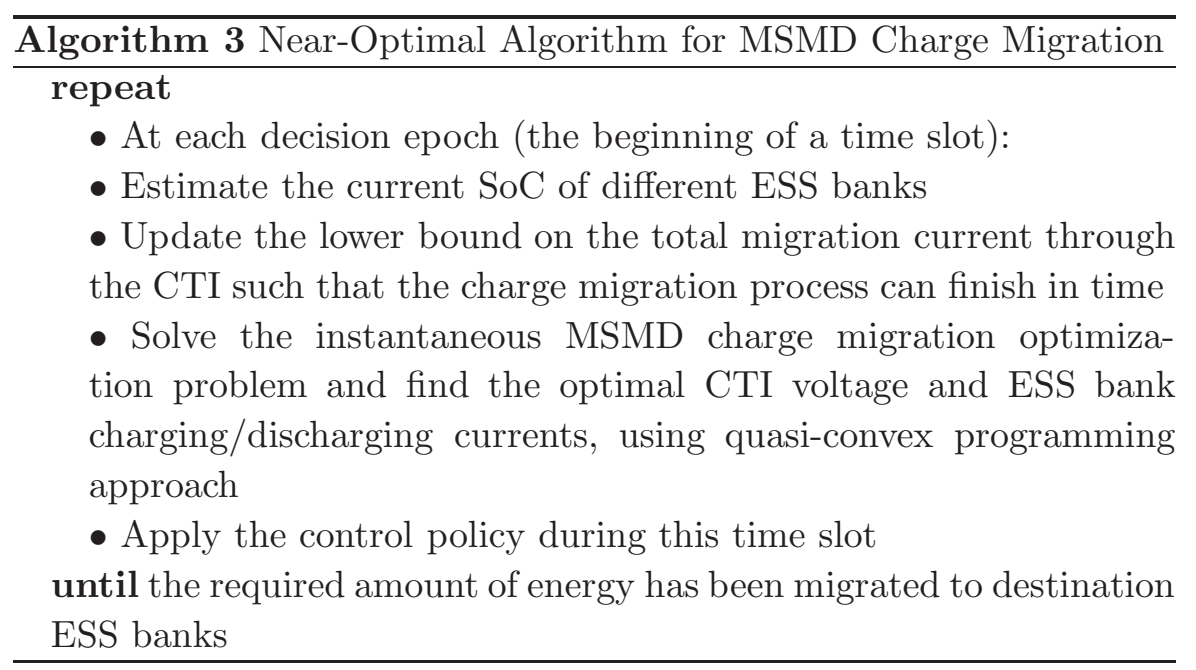



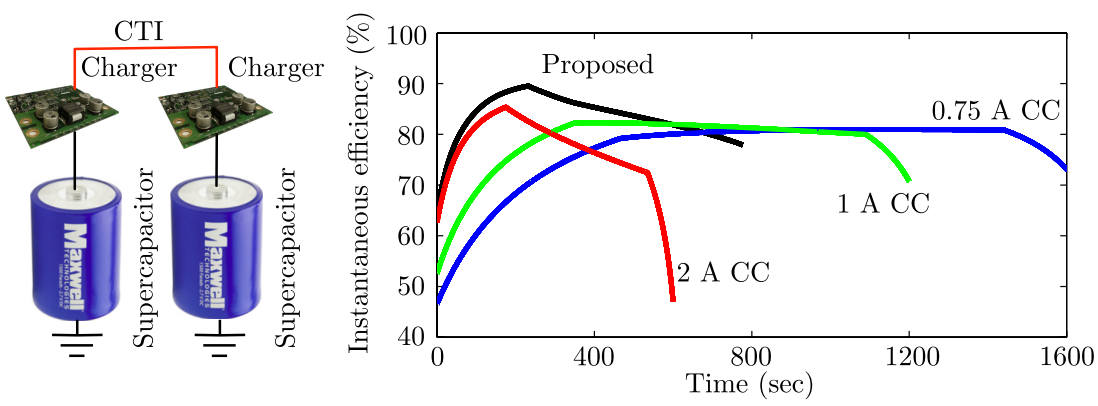

Fig. 5.6 Comparison of charge migration efficiency among different policies. Baselines are $0.75 \mathrm{~A}, 1 \mathrm{~A}$ and $2 \mathrm{~A}$ constant charging current.

Figure 5.6 shows a simple example of charge migration. We transfer 1,200 $\mathrm{C}$ from the source supercapacitor bank to the destination supercapacitor bank without time constraint. We compare the proposed optimal charge migration algorithm with baseline algorithms on charge migration efficiency. The baseline algorithms are constantcurrent charge migration with different destination energy storage bank charging current values. The experimental results show the instantaneous charge migration efficiency during the whole charge migration process. The proposed optimal charge migration algorithm achieves up to $11.6 \%$ efficiency gain compared with baseline setups in this set of experiments.

\subsubsection{Joint Optimization with Power Input and Output}

We expand scope of optimization from HESS to the whole energy system that includes power input (power sources) and output (load devices) in this section. For both the homogeneous and hybrid ESS, joint optimization of the energy storage together with the power input and output design is curial to achieve the true system-wide energy optimality. It becomes more critical when the HESS is used for renewable power sources instead of the power grid with high reliability. We focus on the HESS with renewable energy harvesting especially as an example.

In a storage-equipped renewable energy harvesting system, it is possible and beneficial to generate the maximum amount of power from 
the renewable power sources regardless how much power is needed, consume as much as the load demands, and store the rest in the energy storage for the future use. The maximum power is achieved at a certain voltage and current, which is called the maximum power point (MPP). The MPP dynamically changes due to environmental condition change, and thus we dynamically change the operating voltage and current to remain at the MPP adaptive to the environmental condition change. This technique is called the MPPT, and is considered mandatory for renewable power systems [15, 48, 63].

Figure 5.7 shows power generation, conversion, and storage in a solar energy harvesting system. Typical MPPT techniques perform feedback control of the system so that the power from the PV cell $P_{\mathrm{pv}}$ in Figure 5.7 is maximized. Perturb and observe technique, or hillclimbing method, makes a slight increase or decrease (perturbation) the PV cell voltage $V_{\mathrm{pv}}$ to see which direction increases $P_{\mathrm{pv}}$ (observe), and change $V_{\mathrm{pv}}$ in that way. Incremental conductance technique utilizes that $d P_{\mathrm{pv}} / d V_{\mathrm{pv}}=0$ at the MPP. It compares the incremental conductance $\left(d I_{\mathrm{pv}} / d V_{\mathrm{pv}}\right)$ and the PV cell's instantaneous conductance $\left(I_{\mathrm{pv}} / V_{\mathrm{pv}}\right)$ to determine whether to increase or decrease the $V_{\mathrm{pv}}$ to increase $P_{\mathrm{pv}}$. The common objective of these techniques is to maximize $P_{\mathrm{pv}}$ in a timevarying environmental conditions.

However, it turns out that the MPPT does not guarantee the maximum power production in real practices due to varying power loss $P_{c}$ in the charger. The final amount of power delivered to the energy storage is $P_{\text {charge }}=P_{\mathrm{pv}}-P_{c}$. The MPPT aims at drawing the maximum $P_{\mathrm{pv}}$ without consideration on $P_{c}$. However, $P_{c}$ is not constant but variable

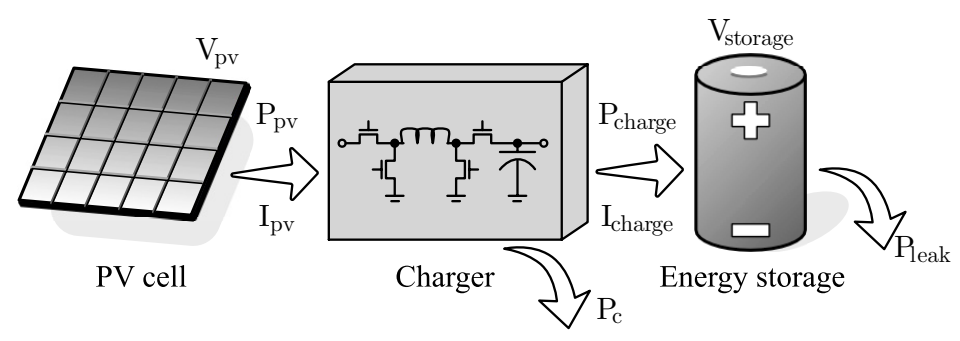

Fig. 5.7 Power transfer and loss in a solar energy harvesting system. 
by the input/output voltage and current of the charger. Consequently, $P_{\text {charge }}$ may not be the maximum if increased $P_{c}$ offsets the increment of $P_{\mathrm{pv}}$ even though the MPPT techniques maximizes it. That is, consideration of both the maximum power extraction from the PV cell/module of the MPPT and minimization of the power loss in there converter are equally important to maximizes PV system efficiency.

In this context, system-level energy efficiency should not be overlooked. Recently, some research in design automation focus on the significant impact of the power conversion efficiency on the systemwide energy efficiency of renewable energy harvesting systems [75, 78, 100, 101, 136, 137, 138. It is shown that the PV cell MPP may not be the same to the system-level MPP in small-scale energy harvesting systems due to significant impact of the power loss in the charger circuit. They presented an energy harvesting system that maximizes the output power of the charge circuit instead of the output power of the energy harvesting power source. Another solar energy harvesting system with charge pump charger is presented with the output power maximization consideration [75].

Systematic optimal design scheme which covers the power source, power converter, and energy storage is first introduced in [78]. There is an attempt in a more recent work to find the optimal size and topology of the energy storage [74]. They examine several supercapacitors of different capacitances to find the best capacitance that maximizes energy efficiency. They also apply reconfiguration of the supercapacitors to dynamically change the terminal voltage and effective capacitance, and take into account the reconfiguration overhead. A cross-layer optimization method derives the optimal design parameters such as PV cell silicon thickness, PV module configuration, and charge pump stage and frequency [114]. A joint optimization method considers the partial shading effect of PV cells for HESS optimization [155]. 


\section{6}

\section{Hybrid Energy Storage System Applications}

\subsection{Residential/Household Applications}

The traditional (static and centrally controlled) structure of the national electricity grid (also known as the power grid) consists of a transmission network, which transmits electrical power generated at remote power plants through long-distance high-voltage power lines to substations, and a distribution network, which delivers electrical power from substations to local end users. In this infrastructure, the local distribution network is often statically adjusted to match the load profile from its end users. The power grid must be able to support the worstcase power demand of all the end users in order to avoid potential power delivery failure as the end user profiles often change drastically according to the day of week and time of day [70].

The smart grid infrastructure is being designed to avoid expending a large amount of capital for increasing the power generation capacity of utility companies in order to meet the expected growth of end user energy consumption at the worst case [8]. The smart grid is integrated with smart meters, which can monitor and control the power flow in the power grid to match the amount of power generation to that of power consumption, and to minimize the overall cost of electrical power 
delivered to the end users. Utility companies can employ dynamic electricity pricing strategies, that is, employing different electricity prices at different time periods in a day or at different locations. This policy will incentivize energy consumers to perform demand side management, also known as demand response, by adjusting their loads to match the current state of the network, that is, shifting their loads from the peak time periods to off-peak periods. There are several ways to perform demand side management, including integration of renewable energy sources such as PV power or wind power at the residential level, demand shaping, household task scheduling, and so on [23].

Although integrating residential-level renewable energy sources into the smart grid proves useful in reducing the usage of fossil fuels, several problems need to be addressed for these benefits to be realized. First, there exists a mismatch between the peak PV power generation time (usually at noon) and the peak load power consumption time for residential users (usually in the evening.) This timing skew results in cases where the generated PV power cannot be optimally utilized for peak power shaving. Moreover, at each time instance, the PV output power is fixed depending on the solar irradiance, when performing the MPPT or MPTT control [78]. Hence, the ability of the residential user for peak shaving is also restricted by the PV output power.

An effective solution of the above-mentioned problems is to incorporate ESS, either homogeneous or hybrid, for houses equipped with PV modules [158]. The proposed residential energy storage stores power from the smart grid during off peak periods of each day and/or from the PV system, and provide power for the end users during the peak periods of that day for peak power shaving and energy cost reduction (since electrical energy tends to be the most expensive during these peak hours.) Therefore, the design of energy pricing-aware control algorithm for the residential storage system, which controls the charging and discharging of energy storage bank(s) and the magnitude of charging/discharing current, is an important task in order for the smart grid technology to deliver on its promises.

Effective storage control algorithms should take into account the realistic electricity pricing function, such as [38, 98]. It consists of both an energy price component, which is a time of usage (TOU) dependent 
function indicating the unit energy price during each time period of the billing period (a day, or a month, and so on), and a demand price component, which is an additional charge due to the peak power consumption in the billing period. The latter component is added to the price of energy consumption in order to prevent a case whereby all the customers utilize their PV power generation and energy storage systems and/or schedule their loads such that a very large amount of power is demanded from the smart grid during low-cost time slots, which can subsequently result in power delivery failure.

Moreover, the size of the ESS is limited due to the relatively high cost of energy storage elements. Therefore, at each decision epoch of a billing period, it is important for the storage controller to forecast the future PV power generation and load power consumption profiles so that it can perform optimization of the total cost. References [29, 62, 159] are representative of general PV power generation and load power consumption predictions by either predicting the whole power profiles, or predicting certain statistical characteristics. Prediction techniques include (but are not limited to): machine learning-based, ant colony clustering-based, and residential activitybased methods. In [158], we propose PV power generation and load power consumption profile predictors specifically designed for the residential ESS controller, that is, exploiting the specific form of the energy price function, and effectively avoiding underestimation of the load power consumption or overestimation of the PV power generation.

Most early work on the residential ESS control and management focuses only on homogeneous systems, and performs battery management without employing systematic optimization and/or optimality consideration in spite of the significant amount of relevant work. For example, reference [118] simply limits the battery current and considers reselling the excessive energy from the PV system to the power grid. Power leveling, which controls the power drawn from the power grid [35], and peak shaving [55] can mitigate this problem, but they do not provide systematic optimization of the system efficiency or the billing cost. Reference [99] provides a systematic optimization based on the Lagrangian relaxation method, but it focuses on issues from the power distribution network such as locational marginal pricing 
(LMP) and transmission congestion problems. Recent work provides an algorithm that determines when and how to charge and discharge the battery, but the method is ad-hoc without much reasoning about the optimally 58.

Based on the PV power generation and load power consumption prediction results, we proposed in [123] a holistic optimization framework for a homogeneous residential ESS, which can effectively mitigate the electricity demand and supply mismatch and minimize the total electricity billing cost. The proposed framework takes into account the PV module impedance, converter loss, battery rate-capacity effect, and storage capacity limit for given solar irradiance profile, load profile, and billing policy. We proposed in 158 a near-optimal residential storage control algorithm under a realistic electricity pricing function with both the energy price component and the demand price component 98. However, we use simpler (and less accurate) models for the ESS in [158] and neglect the conversion power loss. Recent work [82] provides an optimal control algorithm for the residential ESS by assuming that the residential load consumption is task-based and satisfies certain stationary random process, which is known to the storage controller in prior.

HESS provide significant benefits than homogeneous ESS for residential storage, such as higher power capacity, higher energy capacity, faster response time, and longer cycle life, because HESS can exploit the strengths of each type of energy storage element while hiding their weaknesses. Figure 6.1 illustrates the architecture of the proposed HESS for a residential smart grid user with PV power generation. Appropriate control algorithm for a residential-level HESS will be more complicated than the control algorithm for a homogeneous ESS because the former requires taking into account the distinct and complementary characteristics of various types of energy storage elements.

We justify the usage of residential-level HESS in [177]. We consider a HESS comprised of a lithium-ion battery bank and a lead-acid battery bank. We devise an effective control algorithm of both banks in the residential HESS under a simple electricity pricing function [38, as shown in Figure 5.1, in order to minimize the daily energy cost. The electricity pricing function is different during the high season (summer) 


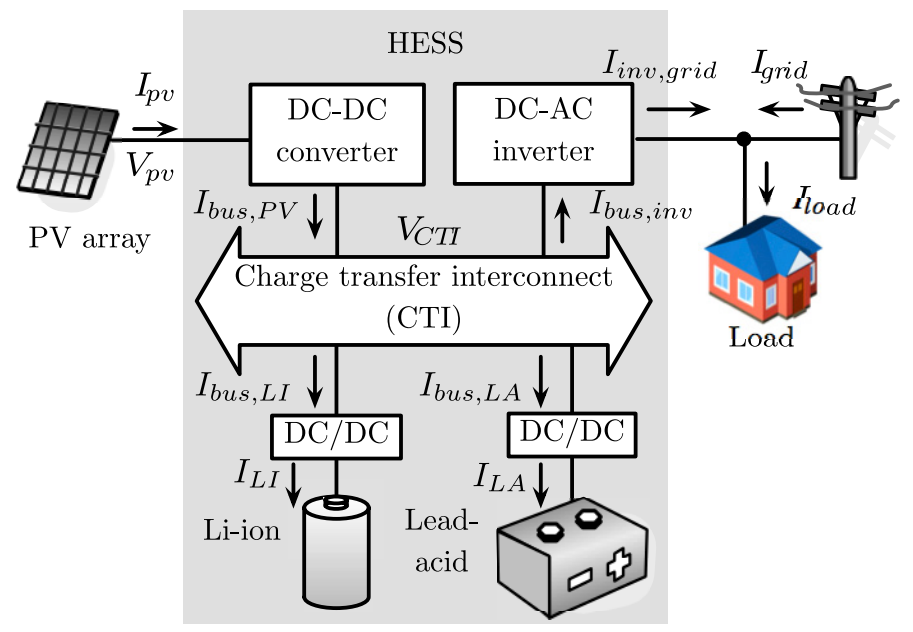

Fig. 6.1 Architecture of the proposed HESS for a residential smart grid user with PV power generation [156].

and low season (winter). Both energy storage banks will get charged during the low peak period and discharged during the high peak period under such electricity pricing function. The optimization variables are the charging/discharging currents of both energy storage banks during these two time periods. We show that the optimally designed HESS achieves an annual return on investment (ROI) of up to $60 \%$ higher than a lead-acid battery-only system or a lithium-ion battery-only system, under the same amount of investment. The HESS is also beneficial in terms of capital cost compared with the homogeneous ESS with the same performance.

In [156], we investigate the problem of optimal control of a HESS for a residential smart grid user equipped with PV power generation facilities. Similar to [177], we consider a HESS comprised of a lithium-ion battery bank and a lead-acid battery bank as an illustration example. We consider a general electricity price function with arbitrary energy price component (we do not consider the demand price in this work), as shown in Figure 6.2 (b). We develop the optimal control algorithm of the residential HESS taking into account the distinct characteristics of different types of energy storage elements, conversion efficiency variations of power converters, as well as the PV module impedance. 

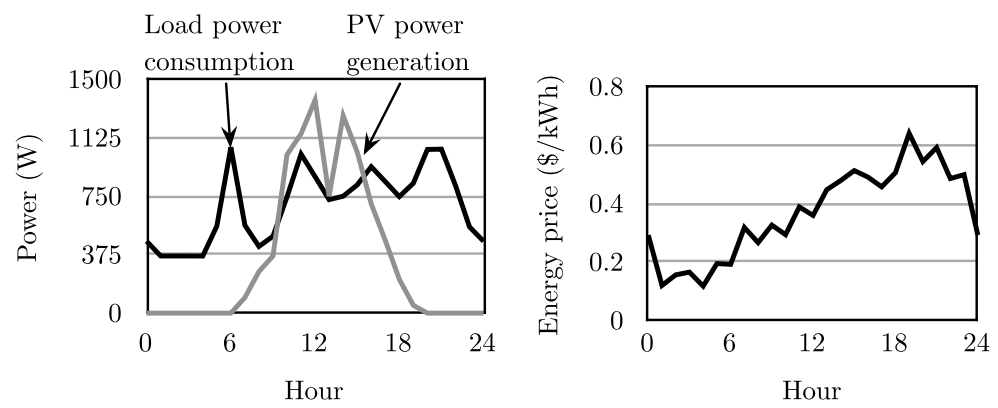

Fig. 6.2 (a) PV power generation and load power consumption profiles and (b) electricity energy price function [156.

The optimal control algorithm is based on the following observation: Suppose that the amounts of energy stored in the lithium-ion bank and in the lead-acid battery are given by $E_{\mathrm{LI}}(t)$ and $E_{\mathrm{LA}}(t)$ at any time $t$ in the optimal solution, the total electricity cost from time $t_{0}$ (the beginning of a day) to time $t$ should be the minimal. The proposed optimal control algorithm is based on dynamic programming and therefore has polynomial time complexity.

Figure 6.3 provides the power profile drawn from the power grid without the HESS where the load power consumption and PV power generation profiles are given in Figure 6.2 (a). It also provides the

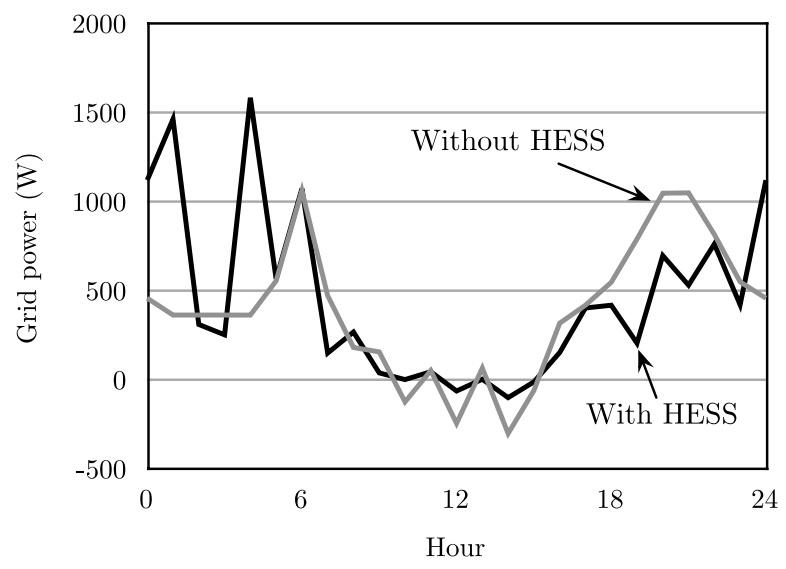

Fig. 6.3 Power drawn from the power grid [156]. 
power profile drawn from the power grid when the HESS and the optimal control algorithm are incorporated. According to Figure 6.3, the HESS could store power from the power grid and the PV system when the electricity price is low, and perform peak shaving when the electricity price is high, thereby reducing the total energy cost over a billing period.

We compare the average profit enhancement between the proposed HESS and the baseline homogenous ESS. We define the amortized cost of an energy storage array, or equivalently, a homogeneous ESS, as the amortized daily capital cost required for purchasing and maintaining the energy storage array. For example, suppose that the capital cost of a lithium-ion battery array is $\$ 3,000$ and its life time is 5 years $(1,825$ days.) The amortized cost is $\$ 3,000 / 1,825$ per day. The amortized cost of a HESS is the sum of the amortized costs of all its energy storage arrays. We define the daily profit of the HESS (or baseline ESS) as the total electricity cost saving in a day due to the incorporation of the HESS (or baseline ESS) minus its amortized cost. We use $\$ 80 / \mathrm{kWh}$ and $\$ 350 / \mathrm{kWh}$ as the unit energy price of lead-acid battery and lithiumion battery, respectively [124. Their lifetimes are 1.5 years and 5 years, respectively [124].

We show the daily profit of the proposed HESS and the two baseline systems over a month of 30 days in Figure 6.4 The HESS outperforms both baseline systems in terms of the daily profit. It achieves an avearge daily profit enhancement of $73.9 \%$ compared with the baseline system consisting of only a lead-acid battery bank due to its relatively low-energy density and charging/discharging performance. The baseline system consisting of only a lithium-ion battery bank even has a negative average daily profit due to its over-high capital cost. We conclude that the HESS is a promising candidate compared to its homogeneous counterparts for residential smart grid usage.

Future work on residential HESS may include (but are not limited to) the following directions:

- The computation complexity of the HESS control algorithm [156] grows exponentially with the increase of the number of energy storage banks. We need to develop effective HESS 


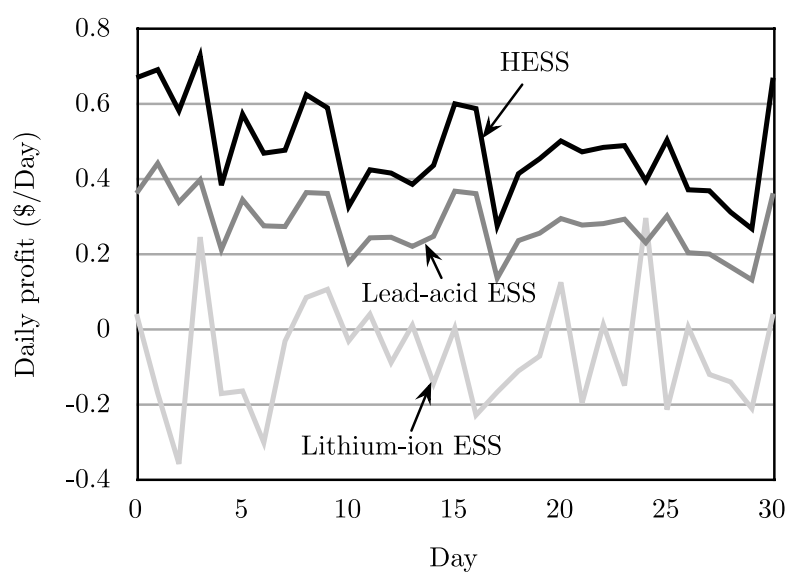

Fig. 6.4 Daily profit of the proposed HESS and two baseline systems [156].

control algorithm that scales well with the increase of energy storage bank count.

- We need to develop HESS control algorithm under both the energy price and the demand price, and compare the performance of the HESS under different electricity pricing scenarios.

- We need to investigate on effective methods to compensate for the errors in PV power generation and load power consumption predictions. We also need to develop effective HESS control algorithms when such prediction results are not available.

\subsection{Electric Vehicle and Hybrid Electric Vehicle Applications}

Automotive research have adopted HESS to improve energy efficiency of the EV/HEV [71]. Supercapacitors are promising energy storage elements to compensate the low rate capability of batteries in EV/HEV. Neural networks may be used to decide the power distribution between the battery and supercapacitors [111, 116] (Figure 6.5). It is reported in [111] that the neural network-based controller achieves more than $20 \%$ of $\mathrm{km} / \mathrm{kWh}$ improvement. Several hybridization topologies and 


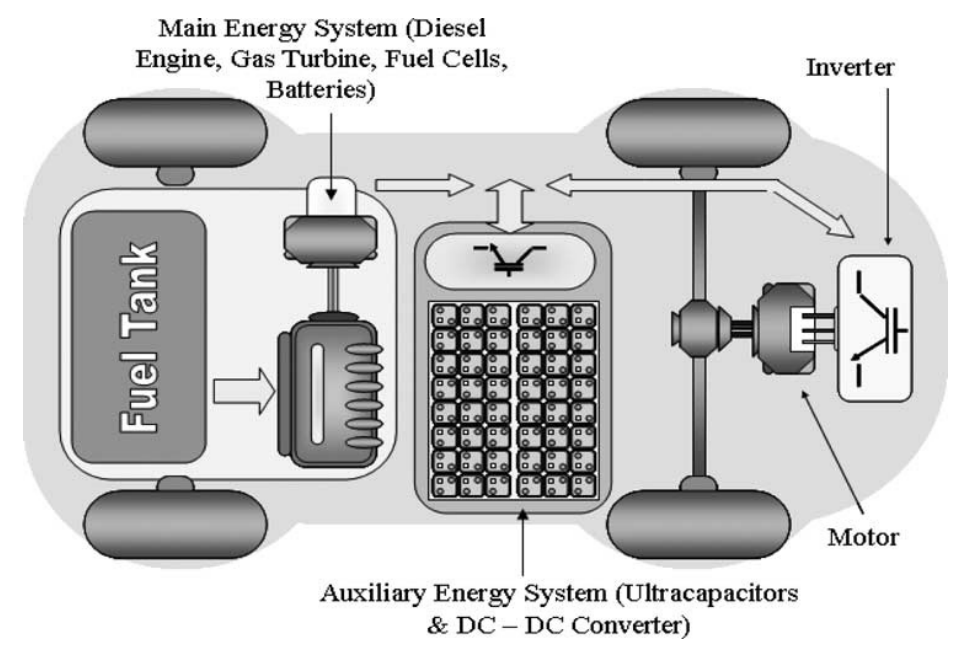

Fig. 6.5 EV/HEV with supercapacitors as auxiliary energy storage [11].

power control methods are studied in [6]. A power control method introduced in [145] is for HEV with a DC-bus composed of a fuel cell, battery, and supercapacitor. A simple passive parallel connection of battery and supercapacitor mitigates voltage ripple with limited volume and weight of the EV/HEV [52]. An optimization method in [129] solves the multi-objective optimization of minimizing energy loss and maximizing power reserve rated power reserve for HEV. Designing highpower, efficient DC-DC converters in limited volume and weight is a big challenge in EV/HEV [116, 135]. Economic viability of using the HESS for EV/HEV is analyzed in [105].

In recent research, it is shown that current distribution while charging and discharging the battery-supercapacitor HESS in an EV requires system-level optimization [25, 122]. They employ charge management schemes introduced in Section [5.2.1, which take account battery rate capability and power conversion efficiency. Figure 6.6 shows the HESS management framework introduced in [122]. It performs charge allocation during regenerative braking, charge replacement during acceleration, and charge migration during idle or cruise period. The amount of energy and deadline of charge transfers are predicted based on map data and GPS navigator. 


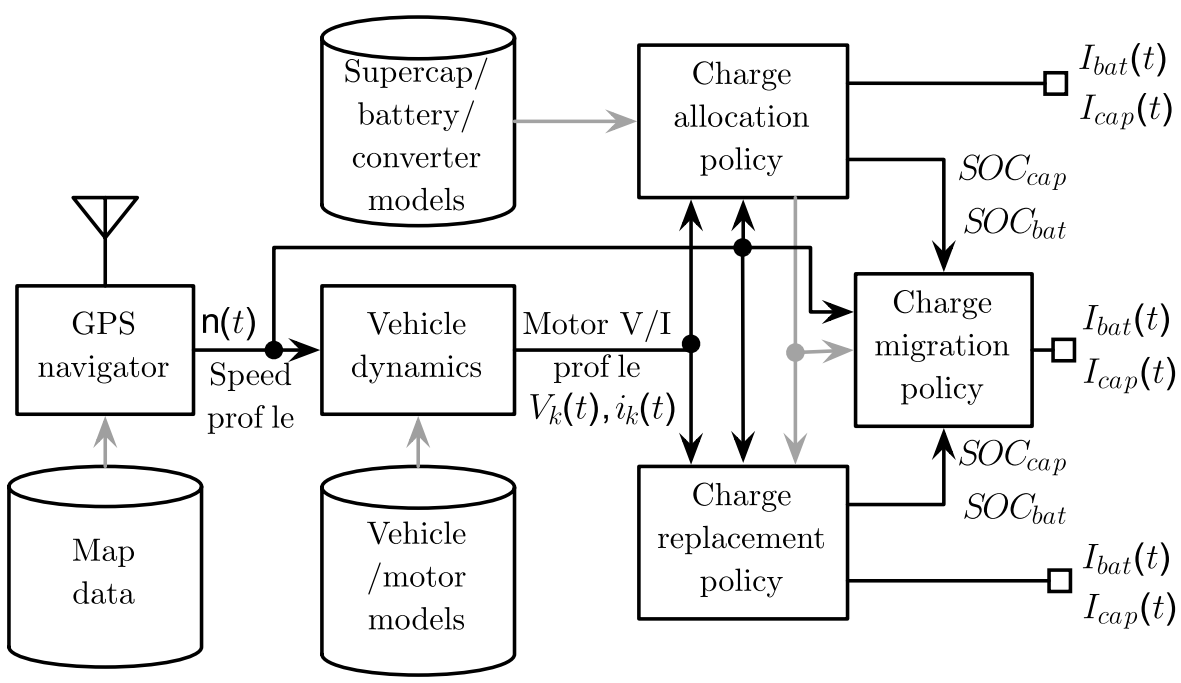

Fig. 6.6 EV HESS management framework [122].

\subsection{Low-power Embedded Systems Applications}

Energy storage for portable electronics have unique requirements compared with ESS for residential or HEV usages. First, it has strict constraints on the size and weight, and requirements for high-energy density per unit volume or unit weight (which is the key criteria for portable ESS.) Hence, Lithium-ion battery is the most promising type for man portable applications nowadays due to its high-energy density. Moreover, some portable applications such as military radios and bio-sensors require small and light form factor, high-energy capacity, and high power capability for a short period of time. Finally, relatively simple structure and control policy are desirable for ESS in portable electronics due to the limited computation capability.

A battery-supercapacitor HESS is a promising candidate to address the above-mentioned requirements. It has a simple architecture and high-energy density due to the usage of Li-ion battery, and can deal with the high-power demand by using supercapacitors as an intermittent energy buffer [139, 140]. We can enhance the total service time of the battery-supercapacitor HESS by use of a constant-current charger circuit compared with a conventional hybrid architecture that simply 


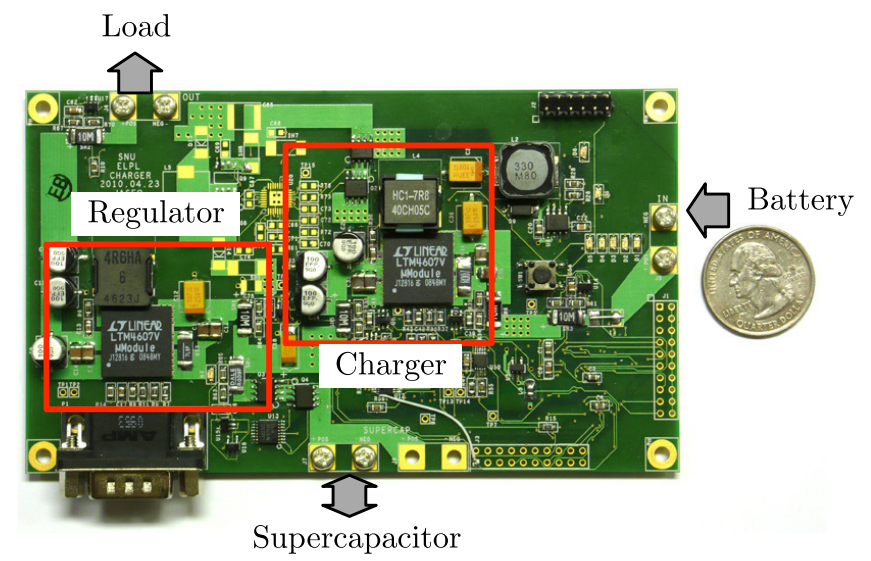

Fig. 6.7 Hybrid power controller board [140].

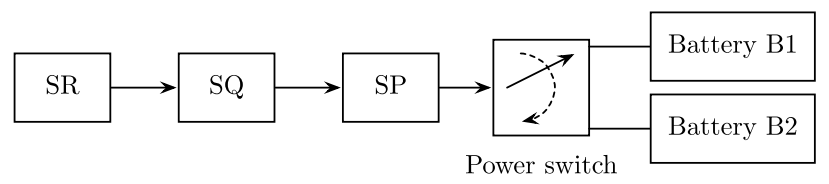

Fig. 6.8 Dual-battery powered portable system model [130].

connects the battery and supercapacitor in parallel. We illustrate the controller board of the HESS in Figure 6.7. The optimization method introduced in [139] derives that a supercapacitor of $2.5 \mathrm{~F}$ capacity maximizes the total service time of the HESS. Experimental results of a real implementation of the constant-current charger-based HESS show $7.7 \%$ total service time improvement. To address a similar problem, early work [130] uses dual-battery as the hybrid power source for a portable electronic system in order for exploiting both the ratecapacity characteristic and the relaxation-induced recovery effect. The block diagram of the portable electronic system is shown in Figure 6.8 This work maximizes the utilization of the battery capacity under a given performance constraint using continuous-time Markov decision process (CTMDP), by modeling the workload arrival times, device service times, and battery selection times as stationary stochastic processes known in prior. However, this assumption may not be realistic. 
As an even more interesting application, battery-supercapacitor HESS can be employed in wireless sensor nodes with energy harvesting in order to significantly extend the life time of the wireless sensors (which is typically restricted by the battery's cycle life) to even achieve near-perpetual operation. Life time extension of the battery is achieved by relying mostly on the supercapacitor as an energy buffer and reducing the frequency in charging/discharging the battery, because the supercapacitor has a nearly infinite cycle life. This is a desirable feature of wireless sensors since frequent replacements of battery may not be an easy task after deployment. Example implementations include [68, 120] as shown in Figure [3.7. The analysis in [68] predicts that the sensor nodes will operate for 43 years under 1\% load, 4 years under $10 \%$ load, and 1 year under $100 \%$ load, even under a simple control algorithm far from optimal. Similar potential applications of HESS include medical devices with energy harvesting from heat or vibration in order to significantly prolong the device's total lifetime. Besides achieving near-perpetual operation, the HESS can reduce the power loss during charge/discharge cycles, which is also critical due to the very limited capability and intermittent nature to produce power from energy harvesting devices such as PV cells. Effective control algorithms should be investigated to take advantage of the high cycle efficiency of the supercapacitor while using the Li-ion battery as a low-leakage long-term energy storage.

Recent work [108, 109, 172] focuses on joint optimization of the embedded electronic system and its hybrid power supply. Reference [108] presents HypoEnergy, a framework for extending the lifetime of the hybrid battery-supercapacitor power supply. HypoEnergy studies the hybrid supply lifetime optimization for a preemptively known workload (a given set of tasks.) The block diagram of HypoEnergy is shown in Figure 6.9. The optimization problem is formulated and mapped to a multiple-choice knapsack problem and solved using the dynamic programming method. The authors evaluate the efficiency and applicability of the HypoEnergy framework using iPhone load measurements. The authors further extend their work to the setup of multiple supercapacitors and workload that is not given a priori [109], and they use the machine learning technique to derive a near-optimal adaptive 


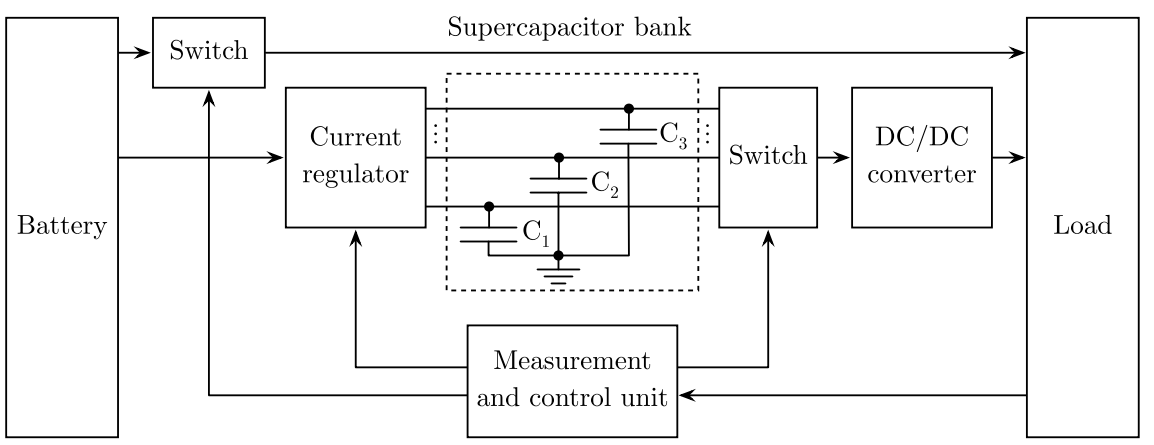

Fig. 6.9 Block diagram of HypoEnergy [108].

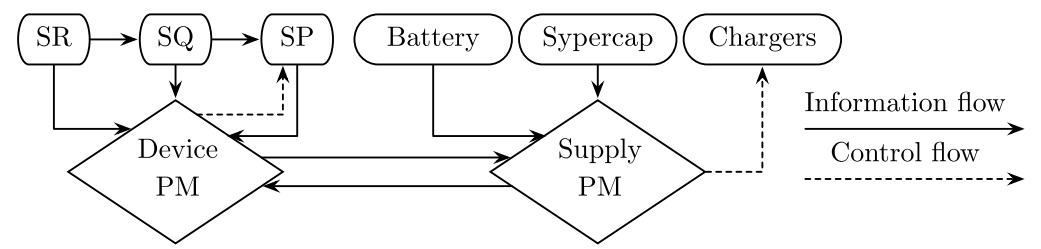

Fig. 6.10 System architecture of the hierarchical reinforcement learning framework with two dedicated PMs [172].

management policy for the hybrid power supply. Recently, we propose to use a model-free reinforcement learning $(\mathrm{RL})$ technique for an adaptive dynamic power management (DPM) framework in embedded systems with bursty workloads, using a hybrid power supply comprised of lithium-ion batteries and supercapacitors [172]. We propose a hierarchical power management framework with two dedicated power managers (PMs): one Supply PM for the hybrid power supply and one Device PM for the embedded device, in order to reduce the online computation overhead. The system and control architecture is shown in Figure 6.10. We use continuous-time Q-learning for the device PM to deal with bursty workloads and use discrete-time Q-learning for the supply PM. The supply PM makes decision at a much lower frequency than the device PM, and they exchange information with each other about embedded system states (such as busy, idle, sleep), number of waiting requests, SoC's of the battery and supercapacitor, and so on. The proposed RL-based DPM approach enhances the power efficiency by up to $9 \%$ compared to a battery-only power supply. 


\section{Conclusions}

The need for high-performance yet cost-effective energy storage system (ESS) is rising as the electrical energy consumption (in many applications) increases and the power generation operating reserve margin becomes tighter. A hybrid ESS (HESS) is an emerging technology, which builds a high-performance and cost-effective ESS with currently available energy storage element technologies. Design and operation of the HESS is far more complex than those for the homogeneous ESS because of the heterogeneity of the energy storage elements. Various benefits for the HESS, such as high power/energy density, low cost, high cycle efficiency, and long cycle life, cannot be achieved unless judicious optimizations are performed during design and operation.

This paper provided an extensive survey of research work and results on key aspects of the HESS, including system architecture, design optimization, and applications. The paper covered the basics of the HESS, starting from the energy storage element technologies and homogeneous ESS to the architecture, optimization schemes, and applications of the HESS in comparison with the homogeneous ESS. 


\section{Acknowledgments}

This work is supported by the National Research Foundation of

Korea (NRF) grant funded by the Korean Government (MEST) (20120005640) and the grants from the Software and Hardware Foundations of the Division of Computer and Communication Foundations of the U.S. National Science Foundation. The ICT at Seoul National University and SPORT lab at University of Southern California provide research facilities for this study. 


\section{References}

[1] "Battery power for your residential solar electric system," Technical Report, National Renewable Energy Laboratory, 2002.

[2] "Electrical energy storage," Technical Report, International Electrochemical Commission, http://www.iec.ch/whitepaper/pdf/iecWP-energystorageLR-en.pdf, 2011.

[3] P. R. Abel, Y.-M. Lin, H. Celio, A. Heller, and C. B. Mullins, "Improving the stability of nanostructured silicon thin film lithium-ion battery anodes through their controlled oxidation," ACS Nano, vol. 6, no. 3, pp. 2506-2516, 2012.

[4] P. R. Abel, Y.-M. Lin, H. Celio, A. Heller, and C. B. Mullins, "Improving the stability of nanostructured silicon thin film lithium-ion battery anodes through their controlled oxidation," ACS Nano, vol. 6, no. 3, pp. 2506-2516, 2012.

[5] H. Akagi and H. Sato, "Control and performance of a doubly-fed induction machine intended for a flywheel energy storage system," IEEE Transactions on Power Electronics, vol. 17, no. 1, pp. 109-116, 2002.

[6] A. Allegre, A. Bouscayrol, and R. Trigui, "Influence of control strategies on battery/supercapacitor hybrid energy storage systems for traction applications," in Proceedings of the Vehicle Power and Propulsion Conference (VPPC), pp. 213-220, 2009.

[7] F. Altaf, L. Johannesson, and B. Egardt, "Performance evaluation of multilevel converter based cell balancer with reciprocating air flow," in Proceedings of the Vehicle Power and Propulsion Conference (VPPC), pp. 706-713, 2012. 
[8] S. M. Amin and B. F. Wollenberg, "Toward a smart grid: power delivery for the 21st century," IEEE Power and Energy Magazine, vol. 3, no. 5, pp. 34-41, 2005.

[9] T. B. Atwater, P. J. Cygan, and F. C. Leung, "Man portable power needs of the 21st century: I. applications for the dismounted soldier. ii. enhanced capabilities through the use of hybrid power sources," Journal of Power Sources, vol. 91, no. 1, pp. 27-36, 2000.

[10] L. Barote, R. Weissbach, R. Teodorescu, C. Marinescu, and M. Cirstea, "Stand-alone wind system with vanadium redox battery energy storage," in Proceedings of the International Conference on Optimization of Electrical and Electronic Equipment (OPTIM), pp. 407-412, 2008.

[11] Y. Barsukov, "Battery cell balancing: What to balance and how," Technical Report, Texas Instruments, 2009.

[12] S. Bashash, S. J. Moura, J. C. Forman, and H. K. Fathy, "Plug-in hybrid electric vehicle charge pattern optimization for energy cost and battery longevity," Journal of Power Sources, vol. 196, no. 1, pp. 541-549, 2011.

[13] F. Belhachemi, S. Raël, and B. Davat, "A physical based model of power electric double-layer supercapacitors," in Proceedings of the Industry Applications Conference, vol. 5, pp. 3069-3076, 2000.

[14] J. L. Bernal-Agustn and R. Dufo-López, "Simulation and optimization of stand-alone hybrid renewable energy systems," Renewable and Sustainable Energy Reviews, vol. 13, no. 8, pp. 2111-2118, 2009.

[15] P. Bhatnagar and R. Nema, "Maximum power point tracking control techniques: State-of-the-art in photovoltaic applications," Renewable and Sustainable Energy Reviews, vol. 23, pp. 224-241, 2013.

[16] A. Bilodeau and K. Agbossou, "Control analysis of renewable energy system with hydrogen storage for residential applications," Journal of Power Sources, vol. 162, no. 2, pp. 757-764, 2006.

[17] S. Boyd and L. Vandenberghe, Convex Optimization. Cambridge University Press, 2004.

[18] D. Bresser, E. Paillard, M. Copley, P. Bishop, M. Winter, and S. Passerini, "The importance of "going nano" for high power battery materials," Journal of Power Sources, vol. 219, pp. 217-222, 2012.

[19] D. A. Brownson, D. K. Kampouris, and C. E. Banks, "An overview of graphene in energy production and storage applications," Journal of Power Sources, vol. 196, no. 11, pp. 4873-4885, 2011.

[20] S. Brutti, J. Hassoun, B. Scrosati, C.-Y. Lin, H. Wu, and H.-W. Hsieh, "A high power Sn-C/C-LiFePO lithium ion battery," Journal of Power Sources, vol. 217, pp. 72-76, 2012.

[21] S. Buller, E. Karden, D. Kok, and R. De Doncker, "Modeling the dynamic behavior of supercapacitors using impedance spectroscopy," in Proceedings of the Industry Applications Conference, vol. 4, pp. 2500-2504, 2001.

[22] S. Buller, M. Thele, R. De Doncker, and E. Karden, "Impedance-based simulation models of supercapacitors and li-ion batteries for power electronic applications," IEEE Transactions on Industry Applications, vol. 41, no. 3, pp. $742-747,2005$. 
[23] S. Caron and G. Kesidis, "Incentive-based energy consumption scheduling algorithms for the smart grid," in Proceedings of the Smart Grid Communications (SmartGridComm) Conference, pp. 391-396, 2010.

[24] P. Carter, J. Baxter, T. Newill, and T. Erekson, "An ultracapacitor-powered race car update," in Proceeding of the Electrical Insulation Conference and Electrical Manufacturing Expo, pp. 267-274, 2005.

[25] S. Chakraborty, M. Lukasiewycz, C. Buckl, S. Fahmy, N. Chang, S. Park, Y. Kim, P. Leteinturier, and H. Adlkofer, "Embedded systems and software challenges in electric vehicles," in Proceedings of the Design, Automation and Test in Europe Conference and Exhibition (DATE), pp. 424-429, 2012.

[26] N. Chang and M. Pedram, "Hybrid electrical energy storage systems," Tutorial at International Symposium on Quality Electronics Design (ISQED), 2011.

[27] N. Chang, J. Seo, D. Shin, and Y. Kim, "Room-temperature fuel cells and their integration into portable and embedded systems," in Proceedings of the Asia and South Pacific Design Automation Conference (ASP-DAC), pp. 69-74, 2010.

[28] P. Chanhom, S. Sirisukprasert, and N. Hatti, "DC-link voltage optimization for SOC balancing control of a battery energy storage system based on a 7-level cascaded PWM converter," in Proceedings of the International Conference on Electrical Engineering/Electronics, Computer, Telecommunications and Information Technology (ECTI-CON), pp. 1-4, 2012.

[29] C. Chen, B. Das, and D. J. Cook, "Energy prediction based on resident's activity," in Proceedings of the International Workshop on Knowledge Discovery from Sensor Data (SensorKDD), 2010.

[30] C.-Y. Chen and P. H. Chou, "DuraCap: a supercapacitor-based, powerbootstrapping, maximum power point tracking energy-harvesting system," in Proceedings of the International Symposium on Low-Power Electronics and Design (ISLPED), pp. 313-318, 2010.

[31] H. Chen, T. N. Cong, W. Yang, C. Tan, Y. Li, and Y. Ding, "Progress in electrical energy storage system: A critical review," Progress in Natural Science, vol. 19, no. 3, pp. 291-312, 2009.

[32] Q. Cheng, J. Tang, J. Ma, H. Zhang, N. Shinya, and L.-C. Qin, "Graphene and carbon nanotube composite electrodes for supercapacitors with ultrahigh energy density," Physical Chemistry Chemical Physics, vol. 13, no. 39, pp. 17615-17624, 2011.

[33] Q. Cheng, J. Tang, J. Ma, H. Zhang, N. Shinya, and L.-C. Qin, "Graphene and nanostructured $\mathrm{MnO}_{2}$ composite electrodes for supercapacitors," Carbon, vol. 49, no. 9, pp. 2917-2925, 2011.

[34] Y. Cheng, V. Joeri, and P. Lataire, "Research and test platform for hybrid electric vehicle with the super capacitor based energy storage," in Proceedings of the European Conference on Power Electronics and Applications, pp. 1-10, 2007.

[35] S. Chiang, K. Chang, and C. Yen, "Residential photovoltaic energy storage system," IEEE Transactions on Industrial Electronics, vol. 45, no. 3, pp. 358-394, 1998. 
[36] Y. Choi, N. Chang, and T. Kim, "DC-DC converter-aware power management for low-power embedded systems," IEEE Transactions on Computer-Aided Design of Integrated Circuits and Systems, vol. 26, no. 8, pp. 1367-1381, 2007.

[37] M. Chowdhury, M. Haque, M. Aktarujjaman, M. Negnevitsky, and A. Gargoom, "Grid integration impacts and energy storage systems for wind energy applications - a review," in Proceedings of the Power and Energy Society General Meeting, pp. 1-8, 2011.

[38] Consolidated Edison Company of New York, Inc. 2012, "Service classification no. 1 - residential and religious,".

[39] A. Czerwiński, S. Obrbowski, and Z. Rogulski, "New high-energy lead-acid battery with reticulated vitreous carbon as a carrier and current collector," Journal of Power Sources, vol. 198, pp. 378-382, 2012.

[40] K. Darcovich, N. Gupta, I. Davidson, and T. Caroni, "Residential electrical power storage scenario simulations with a large-scale lithium ion battery," Journal of Applied Electrochemistry, vol. 40, pp. 749-755, 2010.

[41] J. P. Deane, B. P. Ó. Gallachóir, and E. McKeogh, "Techno-economic review of existing and new pumped hydro energy storage plant," Renewable and Sustainable Energy Reviews, vol. 14, no. 4, pp. 1293-1302, 2010.

[42] K. Divya and J. Østergaard, "Battery energy storage technology for power systems - an overview," Electric Power Systems Research, vol. 79, no. 4, pp. 511-520, 2009.

[43] D. Doerffel and S. A. Sharkh, "A critical review of using the peukert equation for determining the remaining capacity of lead-acid and lithium-ion batteries," Journal of Power Sources, vol. 155, no. 2, pp. 395-400, 2006.

[44] R. Dougal, S. Liu, and R. White, "Power and life extension of batteryultracapacitor hybrids," IEEE Transactions on Components and Packaging Technologies, vol. 25, no. 1, pp. 120-131, 2002.

[45] M. Einhorn, W. Roessler, and J. Fleig, "Improved performance of serially connected li-ion batteries with active cell balancing in electric vehicles," IEEE Transactions on Vehicular Technology, vol. 60, no. 6, pp. 2448-2457, 2011.

[46] O. Ekren and B. Y. Ekren, "Size optimization of a PV/wind hybrid energy conversion system with battery storage using simulated annealing," Applied Energy, vol. 87, no. 2, pp. 592-598, 2010.

[47] O. Ekren, B. Y. Ekren, and B. Ozerdem, "Break-even analysis and size optimization of a $\mathrm{PV} /$ wind hybrid energy conversion system with battery storage - a case study," Applied Energy, vol. 86, no. 78, pp. 1043-1054, 2009.

[48] T. Esram and P. Chapman, "Comparison of photovoltaic array maximum power point tracking techniques," IEEE Transactions on Energy Conversion, vol. 22, no. 2, pp. 439-449, 2007.

[49] A. Evans, V. Strezov, and T. J. Evans, "Assessment of utility energy storage options for increased renewable energy penetration," Renewable and Sustainable Energy Reviews, vol. 16, no. 6, pp. 4141-4147, 2012.

[50] C. Fabjan, J. Garche, B. Harrer, L. Jrissen, C. Kolbeck, F. Philippi, G. Tomazic, and F. Wagner, "The vanadium redox-battery: An efficient storage unit for photovoltaic systems," Electrochimica Acta, vol. 47, no. 5, pp. 825-831, 2001. 
[51] X. Fang, N. Kutkut, J. Shen, and I. Batarseh, "Analysis of generalized parallelseries ultracapacitor shift circuits for energy storage systems," Renewable Energy, 2010.

[52] B. Frenzel, P. Kurzweil, and H. Rönnebeck, "Electromobility concept for racing cars based on lithium-ion batteries and supercapacitors," Journal of Power Sources, vol. 196, no. 12, pp. 5364-5376, 2011.

[53] L. Gao, S. Liu, and R. Dougal, "Dynamic lithium-ion battery model for system simulation," IEEE Transactions on Components and Packaging Technologies, vol. 25, no. 3, pp. 495-505, 2002.

[54] M. Ge, J. Rong, X. Fang, and C. Zhou, "Porous doped silicon nanowires for lithium ion battery anode with long cycle life," Nano Letters, vol. 12, no. 5, pp. 2318-2323, 2012.

[55] F. Giraud and Z. Salameh, "Steady-state performance of a grid-connected rooftop hybrid wind-photovoltaic power system with battery storage," IEEE Transactions on Energy Conversion, vol. 16, no. 1, pp. 1-7, 2001.

[56] M. Glavin and W. Hurley, "Optimisation of a photovoltaic battery ultracapacitor hybrid energy storage system," Solar Energy, vol. 86, no. 10, pp. 30093020, 2012.

[57] P. Guo, H. Song, and X. Chen, "Electrochemical performance of graphene nanosheets as anode material for lithium-ion batteries," Electrochemistry Communications, vol. 11, no. 6, pp. 1320-1324, 2009.

[58] Y. Gurkaynak and A. Khaligh, "Control and power management of a grid connected residential photovoltaic system with plug-in hybrid electric vehicle (PHEV) load," in Proceedings of the Applied Power Electronics Conference and Exposition, pp. 2086-2091, 2009.

[59] G. Gutmann, "Hybrid electric vehicles and electrochemical storage systems a technology pushpull couple," Journal of Power Sources, vol. 84, no. 2, pp. 275279, 1999.

[60] V. Haerri and D. Martinovic, "Supercapacitor module sam for hybrid busses: an advanced energy storage specification based on experiences with the tohycorider bus project," in Proceedings of the Conference of the IEEE Industrial Electronics Society, pp. 268-273, 2007.

[61] W. Henson, "Optimal battery/ultracapacitor storage combination," Journal of Power Sources, vol. 179, no. 1, pp. 417-423, 2008.

[62] T. Hiyama and K. Kitabayashi, "Neural network based estimation of maximum power generation from pv module using environmental information," IEEE Transaction on Energy Conversion, vol. 12, no. 3, pp. 241-247, 1997.

[63] D. P. Hohm and M. E. Ropp, "Comparative study of maximum power point tracking algorithms," Progress in Photovoltaics: Research and Applications, vol. 11, no. 1, pp. 47-62, 2003.

[64] Y. Hou, R. Vidu, and P. Stroeve, "Solar energy storage methods," Industrial and Engineering Chemistry Research, vol. 50, no. 15, pp. 8954-8964, 2011.

[65] A. Imtiaz, F. Khan, and H. Kamath, "A low-cost time shared cell balancing technique for future lithium-ion battery storage system featuring regenerative energy distribution," in Proceedings of the Applied Power Electronics Conference and Exposition (APEC), pp. 792-799, 2011. 
[66] T. Ise, M. Kita, and A. Taguchi, "A hybrid energy storage with a smes and secondary battery," IEEE Transactions on Applied Superconductivity, vol. 15, no. 2, pp. 1915-1918, 2005.

[67] J. Jiang and A. Kucernak, "Electrochemical supercapacitor material based on manganese oxide: Preparation and characterization," Electrochimica Acta, vol. 47, no. 15, pp. 2381-2386, 2002.

[68] X. Jiang, J. Polastre, and D. Culler, "Perpetual environmentally powered sensor networks," in Proceedings of the International Symposium on Information Processing in Sensor Networks (IPSN), pp. 463-468, 2005.

[69] C. Jin, S. Lu, N. Lu, and R. Dougal, "Cross-market optimization for hybrid energy storage systems," in Power and Energy Society General Meeting, 2011 IEEE, pp. 1-6, 2011.

[70] L. D. Kannberg, D. P. Chassin, J. G. DeSteese, S. G. Hauser, M. C. KintnerMeyer, R. G. Pratt, L. A. Schienbein, and W. M. Warwick, "Gridwisetm: The benefits of a transformed energy system," PNNL-14396, Pacific Northwest National Laboratory, 2003.

[71] A. Khaligh and Z. Li, "Battery, ultracapacitor, fuel cell and hybrid energy storage systems for electric, hybrid electric, fuel cell and plug-in hybrid electric vehicles: State of the art," IEEE Transactions on Vehicular Technology, vol. 59, no. 6, pp. 2806-2814, 2010.

[72] H. Kim and K. Shin, "On dynamic reconfiguration of a large-scale battery system," in Proceedings of the Real-Time and Embedded Technology and Applications Symposium (RTAS), pp. 87-96, 2009.

[73] H. Kim and K. Shin, "DESA: Dependable, efficient, scalable architecture for management of large-scale batteries," IEEE Transactions on Industrial Informatics, vol. 8, no. 2, pp. 406-417, 2012.

[74] S. Kim and P. Chou, "Size and topology optimization for supercapacitorbased sub-watt energy harvesters," IEEE Transactions on Power Electronics, vol. 28, no. 4, pp. 2068-2080, 2013.

[75] S. Kim, K.-S. No, and P. Chou, "Design and performance analysis of supercapacitor charging circuits for wireless sensor nodes," IEEE Journal on Emerging and Selected Topics in Circuits and Systems, vol. 1, no. 3, pp. 391-402, 2011.

[76] Y. Kim, S. Park, N. Chang, Q. Xie, Y. Wang, and M. Pedram, "Networked architecture for hybrid electrical energy storage systems," in Proceedings of the Design Automation Conference (DAC), pp. 522-528, 2012.

[77] Y. Kim, S. Park, Y. Wang, Q. Xie, N. Chang, M. Poncino, and M. Pedram, "Balanced reconfiguration of storage banks in a hybrid electrical energy storage system," in Proceedings of the International Conference on ComputerAided Design (ICCAD), pp. 624-631, 2011.

[78] Y. Kim, Y. Wang, N. Chang, and M. Pedram, "Maximum power transfer tracking for a photovoltaic-supercapacitor energy system," in Proceedings of the International Symposium on Low Power Electronics and Design (ISLPED), pp. 307-312, 2010.

[79] J. Kolar, H. Ertl, and F. Zach, "Influence of the modulation method on the conduction and switching losses of a PWM converter system," IEEE Transactions on Industry Applications, vol. 27, no. 6, pp. 1063-1075, 1991. 
[80] F. Koushanfar, "Hierarchical hybrid power supply networks," in Proceedings of the Design Automation Conference (DAC), pp. 629-630, 2010.

[81] F. Koushanfar and A. Mirhoseini, "Hybrid heterogeneous energy supply networks," in Proceedings of the International Symposium on Circuits and Systems (ISCAS), pp. 2489-2492, 2011.

[82] I. Koutsopoulos, V. Hatzi, and L. Tassiulas, "Optimal energy storage control policies for the smart power grid," in Proceedings of the Smart Grid Communications (SmartGridComm) Conference, pp. 475-480, 2011.

[83] R. M. LaFollette and D. N. Bennion, "Design fundamentals of high power density, pulsed discharge, lead-acid batteries. 2. modeling," Journal of the Electrochemical Society, pp. 3701-3707, 1990.

[84] M. LeBreux, M. Lacroix, and G. Lachiver, "Control of a hybrid solar/electric thermal energy storage system," International Journal of Thermal Sciences, vol. 48, no. 3, pp. 645-654, 2009.

[85] K. Lee, N. Chang, J. Zhuo, C. Chakrabarti, S. Kadri, and S. Vrudhula, "A fuel-cell-battery hybrid for portable embedded systems," ACM Transactions on Design Automation of Electronic Systems, vol. 13, no. 1, pp. 19:1-19:34, 2008.

[86] S. Lee, J. Kim, J. Lee, and B. Cho, "State-of-charge and capacity estimation of lithium-ion battery using a new open-circuit voltage versus state-of-charge," Journal of Power Sources, vol. 185, no. 2, pp. 1367-1373, 2008.

[87] T.-Y. Lee and N. Chen, "Determination of optimal contract capacities and optimal sizes of battery energy storage systems for time-of-use rates industrial customers," IEEE Transactions on Energy Conversion, vol. 10, no. 3, pp. 562-568, 1995.

[88] S. Lemofouet and A. Rufer, "A hybrid energy storage system based on compressed air and supercapacitors with maximum efficiency point tracking (mept)," IEEE Transactions on Industrial Electronics, vol. 53, no. 4, pp. 11051115, 2006.

[89] P. Lex and B. Jonshagen, "The zinc/bromine battery system for utility and remote area applications," Power Engineering Journal, vol. 13, no. 3, pp. 142-148, 1999.

[90] C.-H. Li, X.-J. Zhu, G.-Y. Cao, S. Sui, and M.-R. Hu, "Dynamic modeling and sizing optimization of stand-alone photovoltaic power systems using hybrid energy storage technology," Renewable Energy, vol. 34, no. 3, pp. 815-826, 2009.

[91] K. Li, J. Wu, Y. Jiang, Z. Hassan, Q. Lv, L. Shang, and D. Maksimovic, "Large-scale battery system modeling and analysis for emerging electric-drive vehicles," in Proceedings of the International Symposium on Low-Power Electronics and Design (ISLPED), pp. 277-282, 2010.

[92] P. Lian, X. Zhu, S. Liang, Z. Li, W. Yang, and H. Wang, "Large reversible capacity of high quality graphene sheets as an anode material for lithium-ion batteries," Electrochimica Acta, vol. 55, no. 12, pp. 3909-3914, 2010.

[93] D. Linden and T. B. Reddy, "Handbook of Batteries," McGrew-Hill Professional, 2001. 
[94] Linear Technology, "LTC6803-1/LTC6803-3: Multicell battery stack monitor,".

[95] Linear Technology, "LTM4609: $36 \mathrm{~V}_{\text {in }}, 34 \mathrm{~V}_{\text {out }}$ high efficiency buck-boost DC/DC $\mu$ Module regulator,".

[96] C. Liu, Z. Yu, D. Neff, A. Zhamu, and B. Z. Jang, "Graphene-based supercapacitor with an ultrahigh energy density," Nano Letters, vol. 10, no. 12, pp. 4863-4868, 2010.

[97] W. Liu, Y. Wang, W. Liu, Y. Ma, Y. Xie, and H. Yang, "On-chip hybrid power supply system for wireless sensor nodes," in Proceedings of the Asia and South Pacific Design Automation Conference (ASP-DAC), pp. 43-48, 2011.

[98] Los Angeles Department of Water \& Power, Electric Rates, http://www. ladwp.com/ladwp/cms/ladwp001752.jsp.

[99] B. Lu and M. Shahidehpour, "Short-term scheduling of battery in a gridconnected pv/battery system," IEEE Transactions on Power Systems, vol. 20, no. 2, pp. 1053-1061, 2005.

[100] C. Lu, S. P. Park, V. Raghunathan, and K. Roy, "Efficient power conversion for ultra low voltage micro scale energy transducers," in Proceedings of the Design, Automation and Test in Europe Conference and Exhibition (DATE), pp. 1602-1607, 2010.

[101] C. Lu, V. Raghunathan, and K. Roy, "Maximum power point considerations in micro-scale solar energy harvesting systems," in Proceedings of IEEE International Symposium on Circuits and Systems (ISCAS), pp. 273-276, 2010.

[102] S. Lukic, S. Wirasingha, F. Rodriguez, J. Cao, and A. Emadi, "Power management of an ultracapacitor/battery hybrid energy storage system in an HEV," in Proceedings of the Vehicle Power and Propulsion Conference (VPPC), pp. 1-6, 2006.

[103] H. Lund and G. Salgi, "The role of compressed air energy storage (CAES) in future sustainable energy systems," Energy Conversion and Management, vol. 50, no. 5, pp. 1172-1179, 2009.

[104] L. Maharjan, T. Yamagishi, and H. Akagi, "Active-power control of individual converter cells for a battery energy storage system based on a multilevel cascade PWM converter," IEEE Transactions on Power Electronics, vol. 27, no. 3, pp. 1099-1107, 2012.

[105] J. Miller, U. Deshpande, T. Dougherty, and T. Bohn, "Power electronic enabled active hybrid energy storage system and its economic viability," in Proceedings of the IEEE Applied Power Electronics Conference and Exposition (APEC), pp. 190-198, 2009.

[106] J. R. Miller and A. F. Burke., "Electrochemical capacitors: Challenges and opportunities for real-world applications," The Electrochemical Society Interface, vol. 17, no. 1, pp. 53-57, 2008.

[107] A. Millner, "Modeling lithium ion battery degradation in electric vehicles," in Proceedings of IEEE Conference of Innovative Technologies for an Efficient and Reliable Electricity Supply (CITRES), 2010.

[108] A. Mirhoseini and F. Koushanfar, "HypoEnergy. hybrid supercapacitorbattery power-supply optimization for energy efficiency," in Proceedings of the Design, Automation and Test in Europe Conference and Exhibition (DATE), pp. 1-4, 2011. 
[109] A. Mirhoseini and F. Koushanfar, "Learning to manage combined energy supply systems," in Proceedings of the International Symposium on Low-Power Electronics and Design (ISLPED), pp. 229-234, 2011.

[110] S. W. Moore and P. J. Schneider, "A review of cell equalization methods for lithium ion and lithium polymer battery systems," in Proceedings of the SAE 2001 World Congress, pp. 2001-01-0959, 2001.

[111] J. Moreno, M. Ortuzar, and J. Dixon, "Energy-management system for a hybrid electric vehicle, using ultracapacitors and neural networks," IEEE Transactions on Industrial Electronics, vol. 53, no. 2, pp. 614-623, 2006.

[112] T. Morimoto, Y. Che, and M. Tsushima, "Hybrid capacitors using organic electrolytes," Journal of the Korean Chemical Society, vol. 6, no. 3, pp. 174-177, 2003.

[113] S. J. Moura, D. S. Callaway, H. K. Fathy, and J. L. Stein, "Tradeoffs between battery energy capacity and stochastic optimal power management in plug-in hybrid electric vehicles," Journal of Power Sources, vol. 195, no. 9, pp. 29792988, 2010.

[114] E. S. Mungan, C. Lu, V. Raghunathan, and K. Roy, "Modeling, design and cross-layer optimization of polysilicon solar cell based micro-scale energy harvesting systems," in Proceedings of the International Symposium on Low Power Electronics and Design (ISLPED), pp. 123-128, 2012.

[115] T. Nergaard, J. Ferrell, L. Leslie, and J.-S. Lai, "Design considerations for a 48 $\mathrm{v}$ fuel cell to split single phase inverter system with ultracapacitor energy storage," in Proceedings of the Power Electronics Specialists Conference (PESC), vol. 4, pp. 2007-2012, 2002.

[116] M. Ortuzar, J. Moreno, and J. Dixon, "Ultracapacitor-based auxiliary energy system for an electric vehicle: Implementation and evaluation," IEEE Transactions on Industrial Electronics, vol. 54, no. 4, pp. 2147-2156, 2007.

[117] A. Oury, A. Kirchev, Y. Bultel, and E. Chainet, " $\mathrm{PbO}_{2} / \mathrm{Pb}^{2+}$ cycling in methanesulfonic acid and mechanisms associated for soluble lead-acid flow battery applications," Electrochimica Acta, vol. 71, pp. 140-149, 2012.

[118] G. Paloimno, J. Wiles, J. Stevens, and F. Goodman, "Performance of a grid connected residential photovoltaic system with energy storage," in Proceedings of the Photovoltaic Specialists Conference, pp. 1377-1380, 1997.

[119] K. Pan, G. Shi, A. Li, H. Li, R. Zhao, F. Wang, W. Zhang, Q. Chen, H. Chen, Z. Xiong, and D. Finlow, "The performance of a silica-based mixed gel electrolyte in lead acid batteries," Journal of Power Sources, vol. 209, pp. 262-268, 2012.

[120] C. Park and P. Chou, "AmbiMax: Autonomous energy harvesting platform for multi-supply wireless sensor nodes," in Proceedings of the Communications Society Conference on Sensor, Mesh and Ad Hoc Communications and Networks, pp. 168-177, 2006.

[121] C. Park, J. Seo, D. Seo, S. Kim, and B. Kim, "Cost-efficient memory architecture design of nand flash memory embedded systems," in Proceedings of the International Conference on Computer Design, pp. 474-480, 2003.

[122] S. Park, Y. Kim, and N. Chang, "Hybrid energy storage systems and battery management for electric vehicles," in Proceedings of the Design Automation Conference (DAC), pp. 97:1-97:6, 2013. 
[123] S. Park, Y. Wang, Y. Kim, N. Chang, and M. Pedram, "Battery management for grid-connected pv systems with a battery," in Proceedings of the International Symposium on Low-Power Electronics and Design (ISLPED), pp. 115-120, 2012.

[124] M. Pedram, N. Chang, Y. Kim, and Y. Wang, "Hybrid electrical energy storage systems," in Proceedings of the International Symposium on Low-Power Electronics and Design (ISLPED), pp. 363-368, 2010.

[125] V. Presser, C. R. Dennison, J. Campos, K. W. Knehr, E. C. Kumbur, and Y. Gogotsi, "The electrochemical flow capacitor: A new concept for rapid energy storage and recovery," Advanced Energy Materials, vol. 2, no. 7, pp. 895-902, 2012.

[126] H. Qian, J. Zhang, J.-S. Lai, and W. Yu, "A high-efficiency grid-tie battery energy storage system," IEEE Transactions on Power Electronics, vol. 26, no. 3, pp. 886-896, 2011.

[127] F. Rafik, H. Gualous, R. Gallay, A. Crausaz, and A. Berthon, "Frequency, thermal and voltage supercapacitor characterization and modeling," Journal of Power Sources, vol. 165, no. 2, pp. 928-934, 2007.

[128] B. Roberts and J. McDowall, "Commercial successes in power storage," IEEE Power and Energy Magazine, vol. 3, no. 2, pp. 24-30, 2005.

[129] C. Romaus, J. Bocker, K. Witting, A. Seifried, and O. Znamenshchykov, "Optimal energy management for a hybrid energy storage system combining batteries and double layer capacitors," in Proceedings of the Energy Conversion Congress and Exposition (ECCE), pp. 1640-1647, 2009.

[130] P. Rong and M. Pedram, "Battery-aware power management based on markovian decision processes," in Proceedings of the International Conference on Computer-Aided Design (ICCAD), pp. 707-713, 2002.

[131] P. Rong and M. Pedram, "An analytical model for predicting the remaining battery capacity of lithium-ion batteries," IEEE Transactions on Very Large Scale Integration (VLSI) Systems, vol. 14, no. 5, pp. 441-451, 2006.

[132] A. Rufer and P. Barrade, "A supercapacitor-based energy-storage system for elevators with soft commutated interface," IEEE Transactions on Industry Applications, vol. 38, no. 5, pp. 1151-1159, 2002.

[133] M. A. Sakka, H. Gualous, and J. V. Mierlo, "Characterization of supercapacitors matrix," Electrochimica Acta, vol. 55, no. 25, pp. 7532-7537, 2010.

[134] R. Sebastián, F. Yeves, M. Castro, and J. Miguez, "Generalized distributed control system based on can bus for wind diesel hybrid systems," in Proceedings of the International Symposium on Industrial Electronics, vol. 1, pp. 603-608, 2004.

[135] V. Shah, R. Chaudhari, P. Kundu, and R. Maheshwari, "Performance analysis of hybrid energy storage system using hybrid control algorithm with bldc motor driving a vehicle," in Proceedings of the Joint International Conference on Power Electronics, Drives and Energy Systems (PEDES), pp. 1-5, 2010.

[136] H. Shao, C.-Y. Tsui, and W.-H. Ki, "A micro power management system and maximum output power control for solar energy harvesting applications," in Proceedings of the International Symposium on Low Power Electronics and Design (ISLPED), pp. 298-303, 2007. 
[137] H. Shao, C.-Y. Tsui, and W.-H. Ki, "The design of a micro power management system for applications using photovoltaic cells with the maximum output power control," IEEE Transactions on Very Large Scale Integration (VLSI) Systems, vol. 17, no. 8, pp. 1138-1142, 2009.

[138] H. Shao, C.-Y. Tsui, and W.-H. Ki, "Maximizing the harvested energy for micro-power applications through efficient mppt and pmu design," in Proceedings of the Asia and South Pacific Design Automation Conference (ASP-DAC), pp. 75-80, 2010.

[139] D. Shin, Y. Kim, J. Seo, N. Chang, Y. Wang, and M. Pedram, "Batterysupercapacitor hybrid system for high-rate pulsed load applications," in Proceedings of the Design, Automation and Test in Europe Conference and Exhibition (DATE), pp. 1-4, 2011.

[140] D. Shin, Y. Kim, Y. Wang, N. Chang, and M. Pedram, "Constant-current regulator-based battery-supercapacitor hybrid architecture for high-rate pulsed load applications," Journal of Power Sources, vol. 205, pp. 516-524, 2012.

[141] E. Shkolnikov, A. Zhuk, and M. Vlaskin, "Aluminum as energy carrier: Feasibility analysis and current technologies overview," Renewable and Sustainable Energy Reviews, vol. 15, no. 9, pp. 4611-4623, 2011.

[142] F. Simjee and P. Chou, "Everlast: Long-life, supercapacitor-operated wireless sensor node," in Proceedings of the International Symposium on Low-Power Electronics and Design (ISLPED), pp. 197-202, 2006.

[143] S. Teleke, M. Baran, S. Bhattacharya, and A. Huang, "Optimal control of battery energy storage for wind farm dispatching," IEEE Transactions on Energy Conversion, vol. 25, no. 3, pp. 787-794, 2010.

[144] P. Thounthong, V. Chunkag, P. Sethakul, S. Sikkabut, S. Pierfederici, and B. Davat, "Energy management of fuel cell/solar cell/supercapacitor hybrid power source," Journal of Power Sources, vol. 196, no. 1, pp. 313-324, 2011.

[145] P. Thounthong, S. Raël, and B. Davat, "Energy management of fuel cell/battery/supercapacitor hybrid power source for vehicle applications," Journal of Power Sources, vol. 193, no. 1, pp. 376-385, 2009.

[146] R. Torah, P. Glynne-Jones, M. Tudor, T. O'Donnell, S. Roy, and S. Beeby, "Self-powered autonomous wireless sensor node using vibration energy harvesting," Measurement Science and Technology, vol. 19, no. 12, pp. 125202125209, 2008.

[147] M. Uno, "Cascaded switched capacitor converters with selectable intermediate taps for supercapacitor discharger," in Proceedings of the TENCON, pp. 1-5, 2009.

[148] M. Uno, "Series-parallel reconfiguration technique for supercapacitor energy storage systems," in Proceedings of the TENCON, pp. 1-5, 2009.

[149] M. Uno and H. Toyota, "Supercapacitor-based energy storage system with voltage equalizers and selective taps," in Proceedings of the power electronics specialists conference, pp. 755-760, 2008.

[150] S. Vivekchand, C. Rout, K. Subrahmanyam, A. Govindaraj, and C. Rao, "Graphene-based electrochemical supercapacitors," Journal of Chemical Sciences, vol. 120, no. 1, pp. 9-13, 2008. 
[151] S. Vosen and J. Keller, "Hybrid energy storage systems for stand-alone electric power systems: Optimization of system performance and cost through control strategies," International Journal of Hydrogen Energy, vol. 24, no. 12, pp. 1139-1156, 1999.

[152] G. Wang, X. Shen, J. Yao, and J. Park, "Graphene nanosheets for enhanced lithium storage in lithium ion batteries," Carbon, vol. 47, no. 8, pp. 2049-2053, 2009.

[153] H. Wang, L.-F. Cui, Y. Yang, H. Sanchez Casalongue, J. T. Robinson, Y. Liang, Y. Cui, and H. Dai, " $\mathrm{Mn}_{3} \mathrm{O}_{4}$-graphene hybrid as a high-capacity anode material for lithium ion batteries," Journal of the American Chemical Society, vol. 132, no. 40, pp. 13978-13980, 2010.

[154] Y. Wang, Y. Kim, Q. Xie, N. Chang, and M. Pedram, "Charge migration efficiency optimization in hybrid electrical energy storage (HEES) systems," in Proceedings of the International Symposium on Low Power Electronics and Design (ISLPED), pp. 103-108, 2011.

[155] Y. Wang, X. Lin, Y. Kim, N. Chang, and M. Pedram, "Enhancing efficiency and robustness of a photovoltaic power system under partial shading," in Proceedings of the International Symposium on Quality Electronics Design (ISQED), 2012.

[156] Y. Wang, X. Lin, S. Park, N. Chang, and M. Pedram, "Optimal control of a household grid-connected hybrid electrical energy storage system," in Proceedings of the Design, Automation and Test in Europe Conference and Exhibition (DATE), 2013.

[157] Y. Wang, Q. Xie, M. Pedram, Y. Kim, N. Chang, and M. Poncino, "Multiplesource and multiple-destination charge migration in hybrid electrical energy storage systems," in Proceedings of the Design, Automation and Test in Europe Conference and Exhibition (DATE), 2012.

[158] Y. Wang, S. Yue, L. Kerofsky, S. Deshpande, and M. Pedram, "A hierarchical control algorithm for managing electrical energy storage systems in homes equipped with pv power generation," in Proceedings of the Green Technologies Conference, pp. 1-6, 2012.

[159] L. Wei and Z.-H. Han, "Short-term power load forecasting using improved ant colony clustering," in Proceedings of the International Conference on Knowledge Discovery and Data Mining (WKDD), pp. 221-224, 2008.

[160] T.-Y. Wei, C.-H. Chen, H.-C. Chien, S.-Y. Lu, and C.-C. Hu, "A costeffective supercapacitor material of ultrahigh specific capacitances: Spinel nickel cobaltite aerogels from an epoxide-driven sol-gel process," Advanced Materials, vol. 22, no. 3, pp. 347-351, 2010.

[161] R. Wills, J. Collins, D. Stratton-Campbell, C. Low, D. Pletcher, and F. Walsh, "Developments in the soluble lead-acid flow battery," Journal of Applied Electrochemistry, vol. 40, no. 5, pp. 955-965, 2010.

[162] J. Wu, J. Wang, K. Li, H. Zhou, Q. Lv, L. Shang, and Y. Sun, "Large-scale energy storage system design and optimization for emerging electric-drive vehicles," IEEE Transactions on Computer-Aided Design of Integrated Circuits and Systems, vol. 32, no. 3, pp. 325-338, 2013. 
[163] Q. Xie, X. Lin, Y. Wang, M. Pedram, D. Shin, and N. Chang, "State of health aware charge management in hybrid electrical energy storage systems," in Proceedings of the Design, Automation and Test in Europe Conference and Exhibition (DATE), pp. 1060-1065, 2012.

[164] Q. Xie, Y. Wang, Y. Kim, N. Chang, and M. Pedram, "Charge allocation for hybrid electrical energy storage systems," in Proceedings of the International Conference on Hardware/Software Codesign and System Synthesis (CODES+ISSS), pp. 277-284, 2011.

[165] Q. Xie, Y. Wang, Y. Kim, M. Pedram, and N. Chang, "Charge allocation in hybrid electrical energy storage systems," IEEE Transactions on ComputerAided Design of Integrated Circuits and Systems, 2013.

[166] Q. Xie, Y. Wang, Y. Kim, D. Shin, N. Chang, and M. Pedram, "Charge replacement in hybrid electrical energy storage systems," in Proceedings of the Asia and South Pacific Design Automation Conference (ASP-DAC), pp. 627-632, 2012.

[167] Q. Xie, D. Zhu, Y. Wang, Y. Kim, N. Chang, and M. Pedram, "An efficient scheduling algorithm for multiple charge migration tasks in hybrid electrical energy storage systems," in Proceedings of the Asia and South Pacific Design Automation Conference (ASP-DAC), 2013.

[168] A. Xu, S. Xie, and X. Liu, "Dynamic voltage equalization for series-connected ultracapacitors in EV/HEV applications," IEEE Transactions on Vehicular Technology, vol. 58, no. 8, pp. 3981-3987, 2009.

[169] Y. Xue, L. Chang, S. B. Kjaer, J. Bordonau, and T. Shimizu, "Topologies of single-phase inverters for small distributed power generators: an overview," IEEE Transactions on Power Electronics, vol. 19, no. 5, pp. 1305-1314, 2004.

[170] S. Yoda and K. Ishihara, "The advent of battery-based societies and the global environment in the 21st century," Journal of Power Sources, vol. 81-82, pp. 162-169, 1999.

[171] E. Yoo, J. Kim, E. Hosono, H. s. Zhou, T. Kudo, and I. Honma, "Large reversible Li storage of graphene nanosheet families for use in rechargeable lithium ion batteries," Nano Letters, vol. 8, no. 8, pp. 2277-2282, 2008.

[172] S. Yue, D. Zhu, Y. Wang, and M. Pedram, "Reinforcement learning-based dynamic power management in mobile computing systems equipped with hybrid power supply," in Proceedings of IEEE International Conference on Computer Design (ICCD), pp. 81-86, 2012.

[173] C. Zhang, S. Sharkh, X. Li, F. Walsh, C. Zhang, and J. Jiang, "The performance of a soluble lead-acid flow battery and its comparison to a static lead-acid battery," Energy Conversion and Management, vol. 52, no. 12, pp. 3391-3398, 2011.

[174] Y. Zhang, Z. Jiang, and X. Yu, "Control strategies for battery/supercapacitor hybrid energy storage systems," in Proceedings of the IEEE Energy 2030 Conference, pp. 1-6, 2008.

[175] X. Zhao, B. M. Sanchez, P. J. Dobson, and P. S. Grant, "The role of nanomaterials in redox-based supercapacitors for next generation energy storage devices," Nanoscale, vol. 3, pp. 839-855, 2011. 
[176] G. Zhou, D.-W. Wang, F. Li, L. Zhang, N. Li, Z.-S. Wu, L. Wen, G. Q. M. Lu, and H.-M. Cheng, "Graphene-wrapped $\mathrm{Fe}_{3} \mathrm{O}_{4}$ anode material with improved reversible capacity and cyclic stability for lithium ion batteries," Chemistry of Materials, vol. 22, no. 18, pp. 5306-5313, 2010.

[177] D. Zhu, Y. Wang, S. Yue, Q. Xie, N. Chang, and M. Pedram, "Maximizing return on investment of a grid-connected hybrid electrical energy storage system," in Proceedings of Asia and South Pacific Design Automation Conference $(A S P-D A C), 2013$.

[178] J. Zhuo, C. Chakrabarti, and N. Chang, "Energy management of DVS-DPM enabled embedded systems powered by fuel cell-battery hybrid source," in Proceedings of the International Symposium on Low-Power Electronics and Design (ISLPED), pp. 322-327, 2007.

[179] J. Zhuo, C. Chakrabarti, N. Chang, and S. Vrudhula, "Extending the lifetime of fuel cell based hybrid systems," in Proceedings of the Design Automation Conference (DAC), pp. 562-567, 2006.

[180] J. Zhuo, C. Chakrabarti, N. Chang, and S. Vrudhula, "Maximizing the lifetime of embedded systems powered by fuel cell-battery hybrids," in Proceedings of the International Symposium on Low-Power Electronics and Design (ISLPED), pp. 424-429, 2006.

[181] J. Zhuo, C. Chakrabarti, K. Lee, N. Chang, and S. Vrudhula, "Maximizing the lifetime of embedded systems powered by fuel cell-battery hybrids," IEEE Transactions on Very Large Scale Integration (VLSI) Systems, vol. 17, no. 1, pp. 22-32, 2009.

[182] M. Zolot, A. A. Pesaran, and M. Mihalic, "Battery power for your residential solar electric system," Technical Report, National Renewable Energy Laboratory, 2002. 